EUROPEAN JOURNAL OF PURE AND APPLIED MATHEMATICS

Vol.1, No. 1, 2008, (122-196)

ISSN 1307-5543 - www.ejpam.com

Honorary Invited Paper

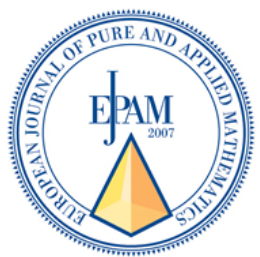

\title{
The Analytic Hierarchy and Analytic Network Measurement Processes: Applications to Decisions under Risk
}

\author{
Thomas L. Saaty ${ }^{1}$ \\ ${ }^{1}$ University of Pittsburgh, Pittsburgh, PA 15260, USA.
}

\begin{abstract}
Mathematics applications largely depend on scientific practice. In science measurement depends on the use of scales, most frequently ratio scales. A ratio scale there is applied to measure various physical attributes and assumes a zero and an arbitrary unit used uniformly throughout an application. Different ratio scales are combined by means of formulas. The formulas apply within structures involving variables and their relations under natural law. The meaning and use of the outcome is then interpreted according to the judgment of an expert as to how well it meets understanding and experience or satisfies laws of nature that are always there. Science derives results objectively, but interprets their significance is subjectively. In decision making, there are no set laws to characterize structures in which relations are predetermined for every decision. Understanding is needed to structure a problem and then also to use judgments to represent importance and preference quantitatively so that a best outcome can be derived by combining and trading off different factors or attributes. From numerical representations of judgments, priority scales are derived and synthesized according to given rules of composition. In decision making the priority scales can only be derived objectively after subjective judgments are made. The process is the opposite of what we do in science. This paper summarizes a mathematical theory of measurement in decision making and applies it to real-life examples of complex decisions.
\end{abstract}

Key words: decision, intangibles, judgments, pairwise comparisons, priorities, synthesis

\section{Introduction}

The Analytic Hierarchy Process (AHP) and its generalization to dependence and feedback, the Analytic Network Process (ANP) are psychophysical theories of measurement. This means that they make the assumption that judgments about subjective feelings and understanding are essentially not very different than and depend on judgments about the physical world in which we acquire our experience and understanding. In the physical world we respond to intensities of occurrence, such as the varying intensities of sight, sound and smell. These intensities fall in different threshold intervals of just noticeable differences because we are unable to detect change in intensity until a stimulus is increased by a noticeable amount. Judgments must reflect not only knowledge about influences, but also the strengths with which these influences occur. These strengths are expressed by us, and especially by experts who have experienced the complexity with which we are concerned, through judgments from which priorities are derived in relative form that reflect numerical intensities that can be validated in those cases where we have

Email address: saaty@katz.pitt.edu (T. Saaty) 
measurement in order to improve our confidence in the applicability of our quantified judgments in those cases where measurements are not available. Measurements in science are made on scales with arbitrary units and need interpretation through judgment to indicate the degree to which they serve our value systems. Occasionally the measurements are used directly in normalized form as priorities that reflect our values if indeed we think they do. In decision making we have to tradeoff different kinds of measurement by filtering them through our judgments from which priorities are derived thereby reducing a multidimensional scaling problem to a one-dimensional scale of priorities of the importance of influences on which our actions are based. All this tells us that it is not enough to advocate the use of a theory with numbers as a justifiable way to make decisions because judgments are subjective any way. There has to be validation of the process through a variety of examples to make it a science based on reason, quantity and mathematics, not a religion based on the strength of authority, belief and lots of statistics devoid of understanding, tradeoffs and interpretation.

To make complex risky decisions we need not only judgments but also structures that represent our best understanding of the flow of influences. The basic structure in doing this is a hierarchy for the AHP and an influence network of clusters and nodes contained within the clusters for the ANP. Priorities are established in the AHP and ANP using pairwise comparisons and judgment. Many decision problems cannot be structured hierarchically because they involve the interaction and dependence of higher-level elements such as objectives and criteria in a hierarchy on lowerlevel elements. Not only does the importance of the criteria determine the importance of the alternatives as in a hierarchy, but also the importance of the alternatives themselves determines the importance of the criteria as in a network Two bridges, both strong, but the stronger is also uglier, would lead one to choose the strong but ugly one unless the criteria themselves are evaluated in terms of the bridges, and strength receives a smaller value and appearance a larger value because both bridges are strong. Feedback enables us to factor the future into the present to determine what we have to do to attain a desired future.

The feedback structure does not have the top-to-bottom form of a hierarchy but looks more like a network, with cycles connecting its components of elements, which we can no longer call levels, and with loops that connect a component to itself. It also has sources and sinks. A source node is an origin of paths of influence (importance) and never a destination of such paths. A sink node is a destination of paths of influence and never an origin of such paths. A full network can include source nodes; intermediate nodes that fall on paths from source nodes, lie on cycles, or fall on paths to sink nodes; and finally sink nodes. Some networks can contain only source and sink nodes. Still others can include only source and cycle nodes or cycle and sink nodes or only cycle nodes. A decision problem involving feedback arises frequently in practice. It can take on the form of any of the networks just described. The challenge is to determine the priorities of the elements in the network and in particular the alternatives of the decision and even more to justify the validity of the outcome. Because feedback involves cycles, and cycling is an infinite process, the operations needed to derive the priorities become more demanding than is with hierarchies.

\section{Paired Comparisons, The fundamental Scale, Eigenvectors, Consistency, HOMOGENEITY}

How to measure intangibles is the main concern of the mathematics of the AHP. In the end we must fit our entire world experience into our system of priorities if we need to understand it in both its details and its general workings. As we said above, the AHP reduces a multidimensional 
problem into a one dimensional one. Decisions are determined by a single number for the best outcome or by a vector of priorities that gives an ordering of the different possible outcomes. We can also combine our judgments or our final choices obtained from a group when we wish to cooperate to agree on a single outcome.

\section{Paired Comparisons and the Fundamental Scale}

To make tradeoffs among the many objectives and criteria, the judgments that are usually made in qualitative terms are expressed numerically. To do this, rather than simply assigning a seemingly arbitrary score out of a person's memory that appears reasonable, one must make reciprocal pairwise comparisons in a carefully designed scientific way. In paired comparisons the smaller or lesser element is used as the unit, and the larger or greater element is estimated as a multiple of that unit with respect to the common property or criterion for which the comparisons are made. In this sense measurement with judgments is made more scientifically than by assigning numbers more or less arbitrarily. Because human beings are limited in size and the firings of their neurons are limited in intensity, it is clear that there is a limit on their ability to compare the very small with the very large. It is precisely for this reason that pairwise comparisons are made on elements or alternatives that are close or homogeneous and the more separated they are, the more need there is to put them in different groups and link these groups with a common element from one group to an adjacent group of slightly greater or slightly smaller elements. In this way one can gradually compare grains of sand of varying sizes increasing to small pebbles and larger stones. When done properly, the largest element in one group is used as the smallest one in the next group, and in the end each group is compared separately and the measurement combined.

From all the paired comparisons, one derives a scale of relative values for the priorities. As we shall see below, due to inevitable inconsistency among the judgments and more importantly because of the need for the invariance of priorities, it is mathematically necessary to derive the priorities in the form of the principal eigenvector of the matrix of paired comparisons.

We learn from making paired comparisons in the AHP that if A is 5 times larger than B and B is 3 times larger than $\mathrm{C}$, then $\mathrm{A}$ is 15 times larger than $\mathrm{C}$ and $\mathrm{A}$ dominates $\mathrm{C} 15$ times. That is different from $\mathrm{A}$ having 5 dollars more than $\mathrm{B}$ and $\mathrm{B}$ having 3 dollars more than $\mathrm{C}$ implies that $\mathrm{A}$ has 8 dollars more than $C$. Defining intensity along the arcs of a graph and raising the matrix to powers measures the first kind of dominance precisely and never the second. It has definite meaning and as we shall see below, in the limit it is measured uniquely by the principal eigenvector. There is a useful connection between what we do with dominance priorities in the AHP and what is done with transition probabilities both of which use matrix algebra to find their answers. Probabilities of transitions between states are multiplied and added. To compose the priorities for the alternatives of a decision with respect to different criteria, it is also necessary that the priorities of the alternatives with respect to each criterion be multiplied by the priority of that criterion and then added over all the criteria.

The Fundamental Scale used for the judgments applied to compare homogeneous (close) elements is given in Table 1. Judgments are first given verbally as indicated in the scale and then a corresponding number is associated with that judgment. 
Table 1 The Fundamental Scale of Absolute Numbers

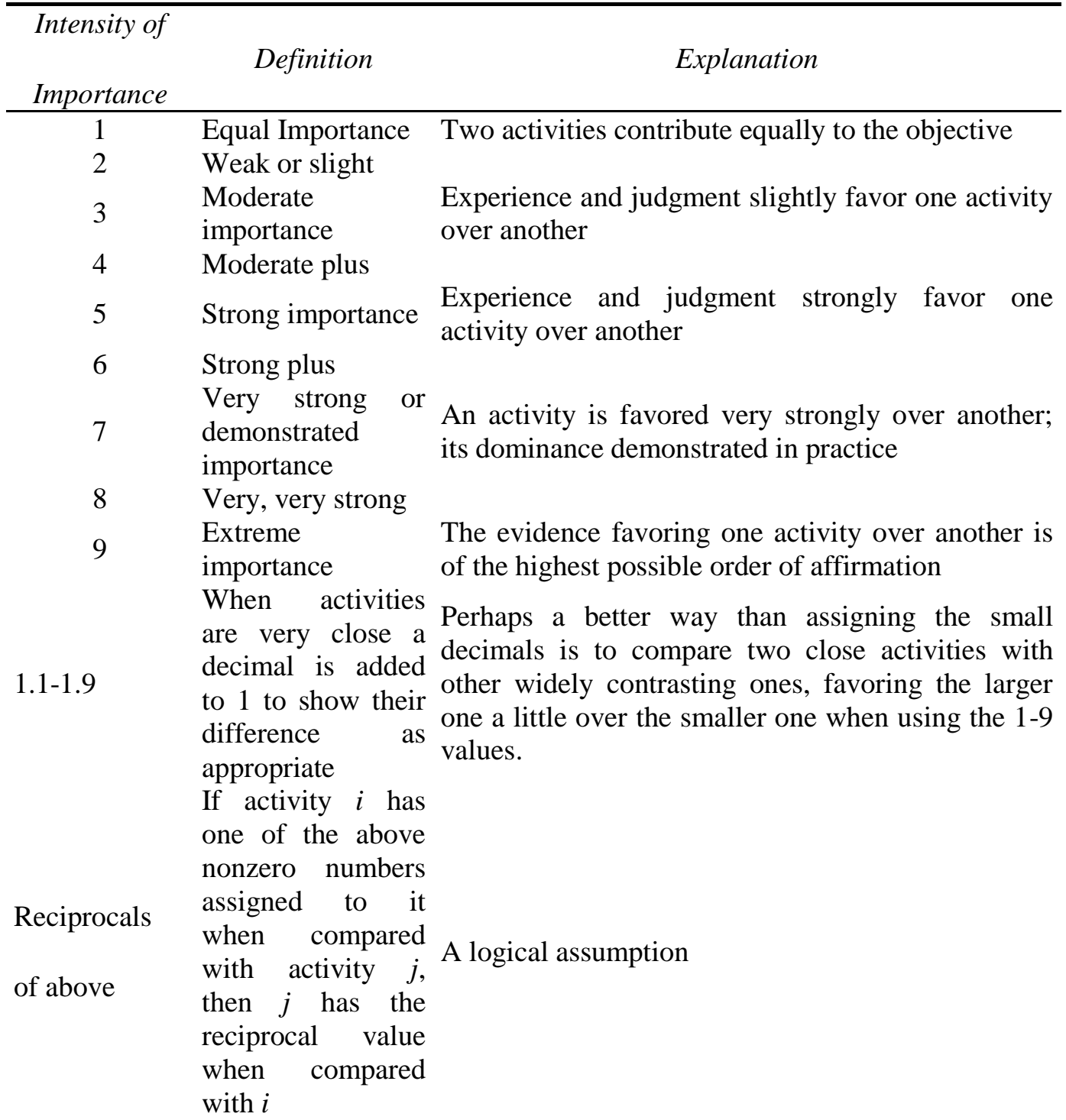

Judgments that represent dominance belong to an absolute scale of numbers which unlike interval and ratio scales that can be transformed to other interval or ratio scales respectively and yield different numbers that mean the same thing, an absolute scale is invariant under the identity transformation that is its numbers cannot be changed to other numbers and mean the same thing. From such numbers priorities can be derived which also belong to an absolute scale of relative numbers whose total sum is equal to one.

Table 2 exhibits an example in which the scale is used to compare the relative consumption of drinks in the United States (done by an audience many years ago). One compares a drink indicated on the left with another indicated at the top and answers the question: How many times 
more, or how strongly more is that drink consumed in the US than the one at the top? More simply which drink of a pair dominates the other and how strongly? In general, one uses the verbal explanation to develop a judgment and then enters its numerical value: for example enter 9 in the (coffee, wine) position meaning that coffee consumption is extremely more than wine consumption. It is automatic that $1 / 9$ is what one needs to use in the (wine, coffee) position. Note that water is consumed a little more than coffee, so one enters 2 in the (water, coffee) position, and $1 / 2$ in the (coffee, water) position. One always enters the whole number in its appropriate position and automatically enters its reciprocal in the transpose position.

The priorities, (obtained in exact form by raising the matrix to large powers and summing each row and dividing each by the total sum of all the rows, or approximately by adding each row of the matrix and dividing by their total and taking the average of the resulting columns) are shown at the bottom of the table along with the true values expressed in relative form by dividing the consumption of each drink (volume) by the sum of the consumption of all drinks. The information about actual consumption was obtained from the US Statistical Abstracts. We see the answers are very close and pair-wise comparison judgments of someone who knows can lead to accurate results of drink consumption. There are numerous examples of this kind of validation.

Table 2 Which Drink is Consumed More in the U.S.?

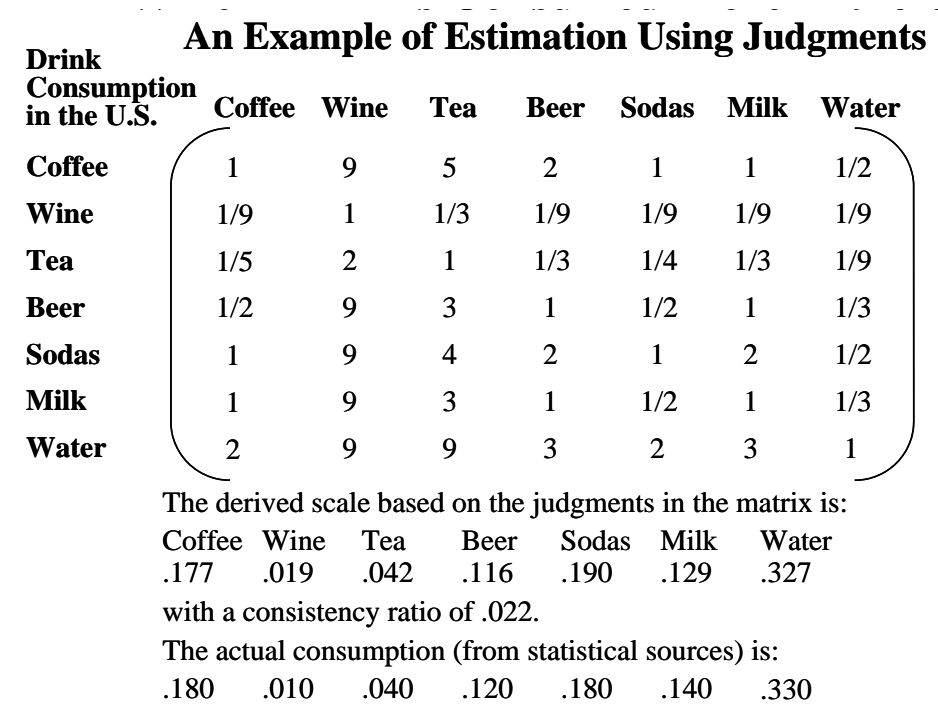

Very early in the history of the subject, T.L. Saaty and M. Khouja did the following exercise on an airplane in 1973. They simply used their common knowledge about the relative influence and standing of these countries in the world and without referring to any specific economic data related to GNP values. The two results are close and demonstrate that the general understanding an interested person has about a problem can be used to advantage to make fairly good estimates through paired comparisons.

Table 3 gives the judgments using the AHP 1-9 scale and Table 4 provides the derived priorities, the actual and relative GNP values.

Table 3 Paired Comparisons of the Relative Dominance in wealth of Seven Nations 


$\left(\begin{array}{cccccccc} & \text { U.S } & \text { U.S.S.R } & \text { China } & \text { France } & \text { U.K } & \text { Japan } & \text { W.Germany } \\ \text { U.S } & 1 & 4 & 9 & 6 & 6 & 5 & 5 \\ \text { U.S.S.R } & 1 / 4 & 1 & 7 & 5 & 5 & 3 & 4 \\ \text { China } & 1 / 9 & 1 / 7 & 1 & 1 / 5 & 1 / 5 & 1 / 7 & 1 / 5 \\ \text { France } & 1 / 6 & 1 / 5 & 5 & 1 & 1 & 1 / 3 & 1 / 3 \\ \text { U.K } & 1 / 6 & 1 / 5 & 5 & 1 & 1 & 1 / 3 & 1 / 3 \\ \text { Japan } & 1 / 5 & 1 / 3 & 7 & 3 & 3 & 1 & 2 \\ \text { W.Germany } & 1 / 5 & 1 / 4 & 5 & 3 & 3 & 1 / 2 & 1\end{array}\right)$

Table 4 Outcome of Estimated Relative Wealth and the Actual and Relative Values

\begin{tabular}{|l|l|l|l|}
\hline & $\begin{array}{l}\text { Normalized } \\
\text { Eigenvector }\end{array}$ & $\begin{array}{l}\text { Actual GNP } \\
(1972)\end{array}$ & $\begin{array}{l}\text { Normalized } \\
\text { GNP Values }\end{array}$ \\
\hline U.S & .427 & 1,167 & .413 \\
\hline U.S.S.R & .23 & 635 & .225 \\
\hline China & .021 & 120 & .043 \\
\hline France & .052 & 196 & .069 \\
\hline U.K & .052 & 154 & .055 \\
\hline Japan & .123 & 294 & .104 \\
\hline W. Germany & .094 & 257 & .091 \\
\hline
\end{tabular}

The reader may now want to know how the foregoing integer-valued scale of response used in making paired comparison judgments can be derived mathematically from the well-known psychophysical logarithmic response function of Weber-Fechner. For a given value of the stimulus, the magnitude of response remains the same until the value of the stimulus is increased sufficiently large in proportion to the value of the stimulus, thus preserving the proportionality of relative increase in stimulus for it to be detectable for a new response. This suggests the idea of just noticeable differences (jnd), well known in psychology.

To derive the values in the scale starting with a stimulus $s_{0}$ successive magnitudes of the new stimuli take the form:

$$
\begin{gathered}
s_{1}=s_{0}+\Delta s_{0}=s_{0}+\frac{\Delta s_{0}}{s_{0}} s_{0}=s_{0}(1+r) \\
s_{2}=s_{1}+\Delta s_{1}=s_{1}(1+r)=s_{0}(1+r)^{2} \equiv s_{0} \alpha^{2} \\
\vdots \\
s_{n}=s_{n-1} \alpha=s_{0} \alpha^{n}(n=0,1,2, \ldots)
\end{gathered}
$$

We consider the responses to these stimuli to be measured on a ratio scale $(b=0)$. A typical response has the form $M_{i}=a \log \alpha^{i}, i=1, \ldots, n$, or one after another they have the form:

$$
M_{1}=a \log \alpha, M_{2}=2 a \log \alpha, \ldots, M_{n}=n a \log \alpha
$$


We take the ratios $M_{i} / M_{1}, \mathrm{i}=1, \ldots, \mathrm{n}$ of these responses in which the first is the smallest and serves as the unit of comparison, thus obtaining the integer values $1,2, \ldots, \mathrm{n}$ of the fundamental scale of the AHP. It appears that numbers are intrinsic to our ability to make comparisons, and that they were not an invention by our primitive ancestors. We must be grateful to them for the discovery of the symbolism. In a less mathematical vein, we note that we are able to distinguish ordinally between high, medium and low at one level and for each of them in a second level below that also distinguish between high, medium and low giving us nine different categories. We assign the value one to (low, low) which is the smallest and the value nine to (high, high) which is the highest, thus covering the spectrum of possibilities between two levels, and giving the value nine for the top of the paired comparisons scale as compared with the lowest value on the scale. Because of increase in inconsistency when we compare more than about 7 elements, we don't need to keep in mind more than $7 \pm 2$ elements. This was first conjectured by the psychologist George Miller in the 1950's. Finally, we note that the scale just derived is attached to the importance we assign to judgments. If we have an exact measurement such as 2.375 and want to use it as it is for our judgment without attaching significance to it, we can use its entire value without approximation.

In the judgment matrix $A$, instead of assigning two numbers $w_{i}$ and $w_{j}$ and forming the ratio $w_{i} / w_{j}$ we assign a single number drawn from the Fundamental Scale of absolute numbers to represent the ratio $\left(w_{i} / w_{j}\right) / 1$. It is a nearest integer approximation to the ratio $w_{i} / w_{j}$. The derived scale will reveal what $w_{i}$ and $w_{j}$ are. This is a central fact about the relative measurement approach. It needs a fundamental scale to express numerically the relative dominance relationship. The general eigenvalue formulation is obtained by perturbation of the following consistent formulation:

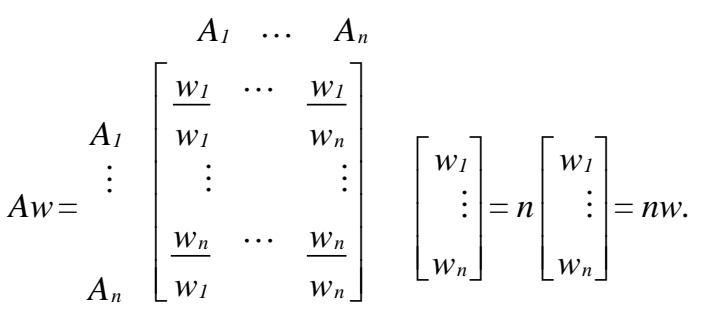

where $A$ has been multiplied on the right by the transpose of the vector of weights $w=\left(w_{1}, \ldots, w_{n}\right)$. The result of this multiplication is $n w$. Thus, to recover the scale from the matrix of ratios, one must solve the problem $A w=n w$ or $(A-n I) w=0$. This is a system of homogeneous linear equations. It has a nontrivial solution if and only if the determinant of $A-n I$ vanishes, that is, $n$ is an eigenvalue of $A$. Now $A$ has unit rank since every row is a constant multiple of the first row. Thus all its eigenvalues except one are zero. The sum of the eigenvalues of a matrix is equal to its trace, that is, the sum of its diagonal elements. In this case the trace of $A$ is equal to $n$. Thus $n$ is an eigenvalue of $A$, and one has a nontrivial solution. The solution consists of positive entries and is unique to within a multiplicative constant. 
The foregoing matrix of ratios of measurements is consistent. Its entries satisfy the relationship $a_{i j} a_{j k}=a_{i k}$ for all $i, j, k$. Note that the ratio of two readings from a ratio scale is an absolute (dimensionless) number. If we were to use judgment we would estimate this absolute number by using the Fundamental Scale of Table 1. When we use judgment we no longer can ensure consistency. It becomes important for us to know how inconsistent we are and which are the most inconsistent judgments and how they can be changed to improve the consistency. But our knowledge may not be adequate to correct our inconsistency as needed. If the inconsistency remains very high despite the changes we make that are compatible with out understanding, we cannot make a decision. The priority weights are obtained directly by adding and normalizing to one the sum of the rows of the matrix, or any of its columns. The intransitivity of influences (how much A dominates B and how much B dominates $\mathrm{C}$ and then how much $\mathrm{C}$ dominates $\mathrm{A}$ ) cannot occur when the judgments are consistent. However, when the judgments are inconsistent, such dominance may happen along with the fact that $a_{i j} a_{j k}=a_{i k}$ for all $i, j, k$ no longer holds. It is known that the different order transitivity of influences can be measured by raising the matrix to different powers. Each power of the matrix yields a set of priorities obtained as the normalized sum of its rows. It is not difficult to show that the average priority of the all these priority vectors is their Cesaro sum that leads to taking the limiting power of the matrix. Perron's theory about positive matrices tells us that this limit is the principal eigenvector of the matrix thus requiring us to solve the principal eigenvalue problem for our positive matrix. This shows that the principal eigenvector is a necessary condition for deriving priorities from inconsistent judgments.

Associated with the weights is an inconsistency index. The consistency index of a matrix is given by. C.I. $=\frac{\lambda_{\max }-n}{n-1} \equiv \mu$. The consistency ratio (C.R.) is obtained by forming the ratio of C.I. and the appropriate one of the following set of numbers shown in Table 5, each of which is an average random consistency index computed for $n \leq 10$ for very large samples. They create randomly generated reciprocal matrices using the scale $1 / 9,1 / 8, \ldots, 1 / 2,1,2, \ldots, 8,9$ and calculate the average of their eigenvalues. This average is used to form the Random Consistency Index R.I. Table 5 shows the values obtained from one set of such simulations and also their first order differences, for matrices of size $1,2, \ldots, 15$. Of course we do not recommend comparing more than 7 items in any single matrix.

Table 5 Random Index

\begin{tabular}{|c|c|c|c|c|c|c|c|c|c|c|c|c|c|c|c|}
\hline Order & 1 & 2 & 3 & 4 & 5 & 6 & 7 & 8 & 9 & 10 & 11 & 12 & 13 & 14 & 15 \\
\hline R.I. & 0 & 0 & 0.52 & 0.89 & 1.11 & 1.25 & 1.35 & 1.40 & 1.45 & 1.49 & 1.52 & 1.54 & 1.56 & 1.58 & 1.59 \\
\hline $\begin{array}{c}\text { First Order } \\
\text { Differences }\end{array}$ & 0 & 0.52 & 0.37 & 0.22 & 0.14 & 0.10 & 0.05 & 0.05 & 0.04 & 0.03 & 0.02 & 0.02 & 0.02 & 0.01 \\
\hline
\end{tabular}

Figure 1 below is a plot of the first two rows of Table 5. It shows the asymptotic nature of random inconsistency. 


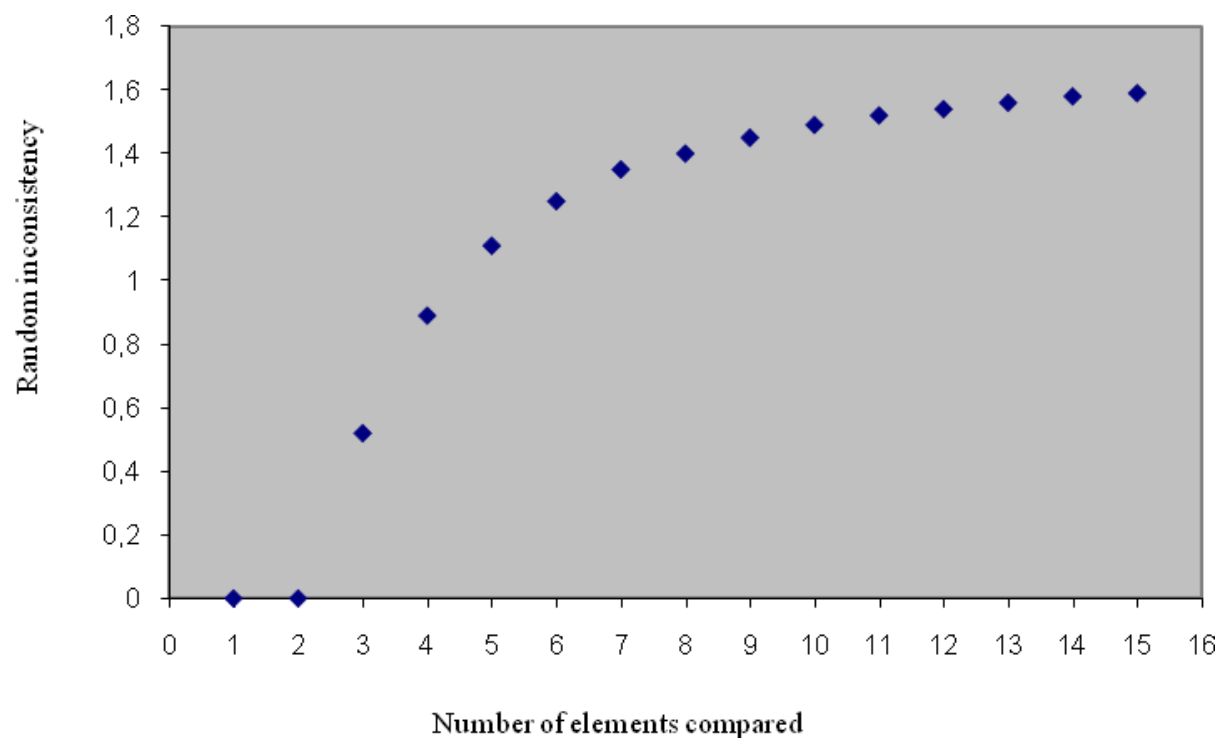

Figu

re 1 Plot of Random Inconsistency

Since it would be pointless to try to discern any priority ranking from a set of random comparison judgments, we should probably be uncomfortable about proceeding unless the consistency index of a pairwise comparison matrix is very much smaller than the corresponding random index value in Table 5. The consistency ratio (C.R.) of a pairwise comparison matrix is the ratio of its consistency index : to the corresponding random index value in Table 5. The notion of order of magnitude is essential in any mathematical consideration of changes in measurement. When one has a numerical value say between 1 and 10 for some measurement and one wishes to determine whether change in this value is significant or not, one reasons as follows: A change of a whole integer value is critical because it changes the magnitude and identity of the original number significantly. If the change or perturbation in value is of the order of a percent or less, it would be so small (by two orders of magnitude) and would be considered negligible. However if this perturbation is a decimal (one order of magnitude smaller) we are likely to pay attention to modify the original value by this decimal without losing the significance and identity of the original number as we first understood it to be. Thus in synthesizing near consistent judgment values, changes that are too large can cause dramatic change in our understanding, and values that are too small cause no change in our understanding. We are left with only values of one order of magnitude smaller that we can deal with incrementally to change our understanding. It follows that our allowable consistency ratio should be not more than about .10 for a matrix larger than 5 by $5,8 \%$ for a 4 by 4 matrix and $5 \%$ for a 3 by 3 matrix. This requirement cannot be made smaller such as $1 \%$ or $.1 \%$ without trivializing the impact of inconsistency. But inconsistency itself is important because without it, new knowledge that changes preference cannot be admitted. Assuming that all knowledge should be consistent contradicts experience that requires continued revision of understanding.

If the C.R. is larger than desired, we do three things: 1) Find the most inconsistent judgment in the matrix (for example, that judgment for which $\varepsilon_{i j}=a_{i j} w_{j} / w_{i}$ is largest), 2) 
Determine the range of values to which that judgment can be changed corresponding to which the inconsistency would be improved, 3) Ask the judge to consider, if he can, change his judgment to a plausible value in that range. If he is unwilling, we try with the second most inconsistent judgment and so on. If no judgment is changed the decision is postponed until better understanding of the stimuli is obtained. Judges who understand the theory are always willing to revise their judgments often not the full value but partially and then examine the second most inconsistent judgment and so on. It can happen that a judge's knowledge does not permit the improvement of consistency and more information is required to improve that consistency.

Before proceeding further, the following observations may be useful for a better understanding of the importance of the concept of a limit on our ability to process information and also change in information. The quality of response to stimuli is determined by three factors. Accuracy or validity, consistency, and efficiency or amount of information generated. Our judgment is much more sensitive and responsive to large perturbations. When we speak of perturbation, we have in mind numerical change from consistent ratios obtained from priorities. The larger the inconsistency and hence also the larger the perturbations in priorities, the greater is our sensitivity to make changes in the numerical values assigned. Conversely, the smaller the inconsistency, the more difficult it is for us to know where the best changes should be made to produce not only better consistency but also better validity of the outcome. Once near consistency is attained, it becomes uncertain which coefficients should be perturbed by small amounts to transform a near consistent matrix to a consistent one. If such perturbations were forced, they could be arbitrary and thus distort the validity of the derived priority vector in representing the underlying decision.

The third row of Table 5 gives the differences between successive numbers in the second row. Figure 2 is a plot of these differences and shows the importance of the number seven as a cutoff point beyond which the differences are less than 0.10 where we are not sufficiently sensitive to make accurate changes in judgment on several elements simultaneously. A similar argument plot be made by using the ratios of the numbers in the third row of Table 5 for $n \geq 3$.

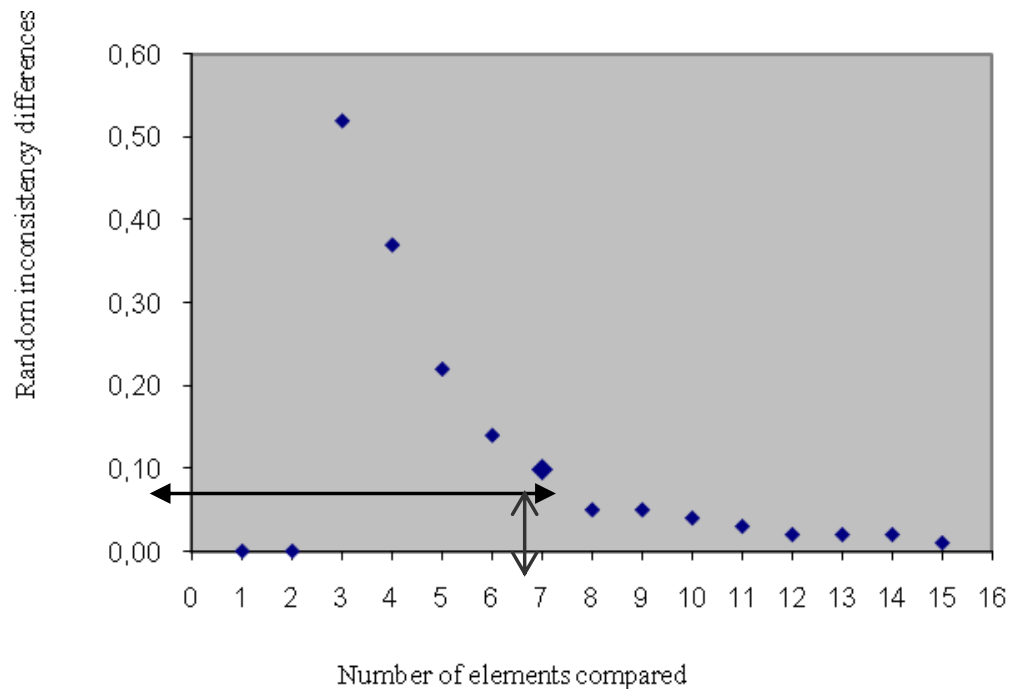

Figure 2 Plot of First Differences in Random Inconsistency 


\section{Homogeneity}

Homogeneity as an important concept to ensure consistency in the paired comparisons requires the elements to be of the same order of magnitude which means that our perceptions in comparing them should be of nearly the same order of magnitude. It is a fact that people are unable to directly compare widely disparate objects such as a ping-pong ball and a basketball according to volume. To do that, we need a range greater than the 1-9 scale. To resolve this problem, we can use a method in which we cluster different elements so we can rate them within a cluster and then rate them across the clusters. We need to add other objects to make the comparison possible and then form groups of comparable elements. A common element, the pivot, could be the largest in one cluster and the smallest in the next cluster of the next higher order of magnitude. The weights of the elements in the second group are divided by the priority of the pivot in that group and then multiplied by the priority of the same pivot element from the first group, making them comparable with the first group. The process is then continued.

Table 6 shows how this process works in comparing a cherry tomato with a water melon, which appears to be two orders of magnitude bigger in size, by introducing intermediate objects in stages.

Table 6 Comparisons According to Volume

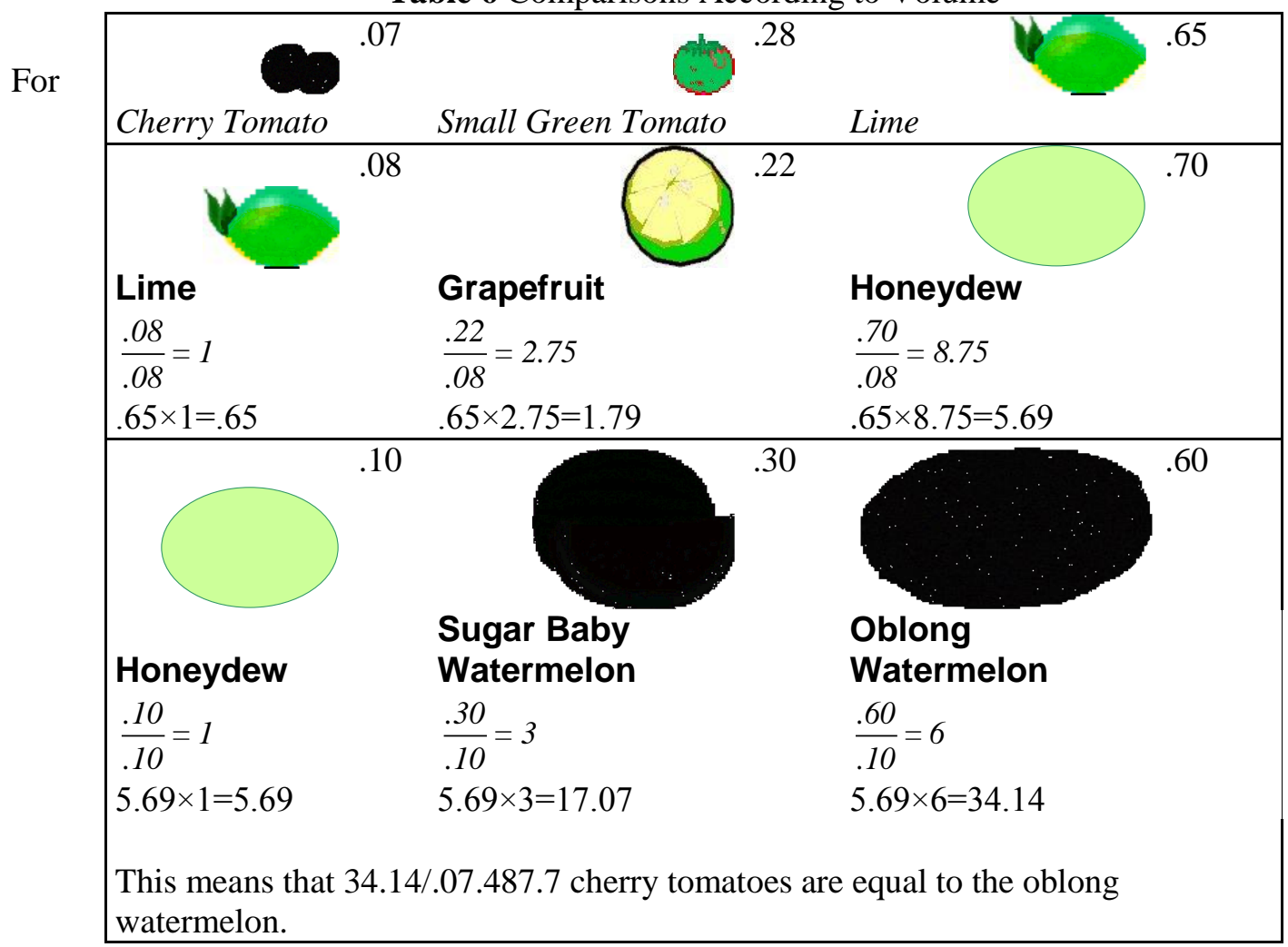

given positive reciprocal matrix $A=\left[a_{i j}\right]$ and a given pair of distinct indices $k>l$, define $A(t)=$ $\left[a_{i j}(t)\right]$ by $a_{k l}(t) \equiv a_{k l}+t, a_{l k}(t) \equiv\left(a_{l k}+t\right)^{-1}$, and $a_{i j}(t) \equiv a_{i j}$ for all $i \neq k, j \neq l$, so $A(0)=A$. Let $\lambda_{\text {max }}(t)$ denote the Perron eigenvalue of $A(t)$ for all $t$ in a neighborhood of $t=0$ that is small enough to ensure that all entries of the reciprocal matrix $A(t)$ are positive there. Finally, let $v=\left[v_{i}\right]$ be the 
unique positive eigenvector of the positive matrix $A^{T}$ that is normalized so that $v^{T} w=1$. Then a classical perturbation formula tells us that

$$
\left.\frac{d \lambda_{\max }(t)}{d t}\right|_{t=0}=\frac{v^{T} A^{\prime}(0) w}{v^{T} w}=v^{T} A^{\prime}(0) w=v_{k} w_{l}-\frac{1}{a_{k l}^{2}} v_{l} w_{k}
$$

We conclude that

$$
\frac{\partial \lambda_{\max }}{\partial a_{i j}}=v_{i} w_{j}-a_{j i}{ }^{2} v_{j} w_{i} \text { for all } i, j=1, \ldots, n \text {. }
$$

Because we are operating within the set of positive reciprocal matrices, $\frac{\partial \lambda_{\max }}{\partial a_{j i}}=-\frac{\partial \lambda_{\max }}{\partial a_{i j}}$ for all $i$ and $j$. Thus, to identify an entry of $A$ whose adjustment within the class of reciprocal matrices would result in the largest rate of change in $\lambda_{\max }$ we should examine the $n(n-1) / 2$ values $\left\{v_{i} w_{j}-a_{j i}{ }^{2} v_{j} w_{i}\right\}, i>j$ and select (any) one of largest absolute value.

\section{ADDITIVE COMPOSITION IS NECESSARY}

Sometimes people have assigned criteria different weights when they are measured in the same unit. Others have used different ways of synthesis than multiplying and adding. An example should clarify what we must do. Synthesis in the AHP involves weighting the priorities of elements compared with respect to an element in the next higher level, called a parent element, by the priority of that element and adding over all such parents for each element in the lower level. Consider the example of two criteria $C_{1}$ and $C_{2}$ and three alternatives $A_{1}, A_{2}$ and $A_{3}$ measured in the same scale such as dollars. If the criteria are each assigned the value 1, then the weighting and adding process produces the correct dollar value as in Table 7.

Table 7 Calculating Returns Arithmetically

\begin{tabular}{l|c|c|c|c|}
\cline { 2 - 5 } & $\begin{array}{c}\text { Criterion } \mathrm{C}_{1} \\
\text { Unnormalized } \\
\text { weight }=1.0\end{array}$ & $\begin{array}{c}\text { Criterion } \mathrm{C}_{2} \\
\text { Unnormalized } \\
\text { weight }=1.0\end{array}$ & $\begin{array}{c}\text { Weighted Sum } \\
\text { Unnormalized }\end{array}$ & $\begin{array}{c}\text { Normalized or } \\
\text { Relative values }\end{array}$ \\
\cline { 2 - 5 } $\mathrm{A}_{1}$ & 200 & 150 & 350 & $350 / 1300=.269$ \\
$\mathrm{~A}_{2}$ & 300 & 50 & 350 & $350 / 1300=.269$ \\
$\mathrm{~A}_{3}$ & 500 & 100 & 600 & $600 / 1300=.462$ \\
$\begin{array}{l}\text { Column } \\
\text { totals }\end{array}$ & 1000 & 300 & 1300 & 1 \\
\cline { 2 - 5 } & & & & \\
\cline { 2 - 5 } & & &
\end{tabular}

However, it does not give the correct outcome if the weights of the criteria are normalized, with each criterion having a weight of .5. Once the criteria are given in relative terms, so must the alternatives also be given in relative terms. A criterion that measures values in pennies cannot be as important as another measured in thousands of dollars. In this case, the only meaningful 
importance of a criterion is the ratio of the total money for the alternatives under it to the total money for the alternatives under both criteria. By using these weights for the criteria, rather than .5 and .5 , one obtains the correct final relative values for the alternatives.

What is the relative importance of each criterion? Normalization indicates relative importance. Relative values require that criteria be examined as to their relative importance with respect to each other. What is the relative importance of a criterion, or what numbers should the criteria be assigned that reflect their relative importance? Weighting each criterion by the proportion of the resource under it, as shown below in Table 8, and multiplying and adding as in the additive synthesis of the AHP, we get the same correct answer. For criterion $\mathrm{C}_{1}$ we have

$(200+300+500) /[(200+300+500)+(150+50+100)=1000 / 1300$

and for criterion $\mathrm{C}_{2}$ we have $(150+50+100) /[(200+300+500)+(150+50+100)=300 / 1300$.

Here the criteria are automatically in normalized form, and their weights sum to one. We see that when the criteria are normalized, the alternatives must also be normalized to get the right answer. For example, if we look in Table 7 we have $350 / 1300$ for the priority of alternative $A_{1}$. Now if we simply weight and add the values for alternative $A_{1}$ in Table 8 we get for its final value $(200 / 1000)(1000 / 1300)+(150 / 300)(300 / 1300)=350 / 1300$ which is the same as in Table 7 . It is clear that if the priorities of the alternatives are not normalized one does not get the desired outcome.

Table 8 Normalized Criteria Weights and Normalized Alternative Weights From Measurements in the Same Scale (Additive Synthesis)

\begin{tabular}{l|c|c|c|}
\cline { 2 - 4 } Alternatives & $\begin{array}{c}\text { Criterion } \mathrm{C}_{1} \\
\text { Normalized weight }= \\
1000 / 1300=0.7692\end{array}$ & $\begin{array}{c}\text { Criterion } \mathrm{C}_{2} \\
\text { Normalized weight= } \\
300 / 1300=0.2308\end{array}$ & Weighted Sum \\
\hline $\mathrm{A}_{1}$ & $200 / 1000$ & $150 / 300$ & $350 / 1300=.2692$ \\
$\mathrm{~A}_{2}$ & $300 / 1000$ & $50 / 300$ & $350 / 1300=.2692$ \\
\hline $\mathrm{A}_{3}$ & $500 / 1000$ & $100 / 300$ & $600 / 1300=.4615$ \\
\hline
\end{tabular}

We have seen in this example that in order to obtain the correct final relative values for the alternatives when measurements on a measurement scale are given, it is essential that the priorities of the criteria be derived from the priorities of the alternatives. Thus when the criteria depend on the alternatives we need to normalize the values of the alternatives to obtain the final result. This procedure is known as the distributive mode of the AHP. It is also used in case of functional (real life not paired comparison) dependence of the alternatives on the alternatives and of the criteria on the alternatives. The AHP is a special case of the Analytic Network Process. The dominant mode of synthesis in the ANP with all its interdependencies is the distributive mode. The ANP automatically assigns the criteria the correct weights, if one only uses the normalized values of the alternatives under each criterion and also the normalized values for each alternative under all the criteria without any special attention to weighting the criteria.

\section{Benefits, Opportunities, Costs and Risks}

The process of decision-making requires us to analyze a decision according to Benefits (B), the good things that would result from taking the decision; Opportunities $(\mathrm{O})$, the potentially good things that can result in the future from taking the decision; Costs (C), the pains and 
disappointments that would result from taking the decision; and Risks (R), the potential pains and disappointments that can result from taking the decision. We then create control criteria and subcriteria or even a network of criteria under each and develop a subnet and its connection for each control criterion. Next we determine the best outcome for each control criterion and combine the alternatives in what is known as the ideal form for all the control criteria under each of the BOCR merits. Then we take the best alternative under B and use it to think of benefits and the best one under $\mathrm{O}$, which may be different than the one under $\mathrm{C}$, and use it to think of opportunities and so on for costs and risks. Finally we must rate these four with respect to the strategic criteria (criteria that underlie the evaluations of the merits all the decisions we make) using the ratings mode of the AHP to obtain priority ratings for $\mathrm{B}, \mathrm{O}, \mathrm{C}$, and $\mathrm{R}$. We then normalize (not mandatory but recommended) and use these weights to combine the four vectors of outcomes for each alternative under BOCR to obtain the overall priorities. We can form the ratio $\mathrm{BO} / \mathrm{CR}$ which does not need the BOCR ratings to obtain marginal overall outcomes. Alternatively and better, 1) we can use the ratings to weight and subtract the costs and risks from the sum of the weighted benefits and opportunities.

\section{HIERARCHIES}

Structuring a complex decision is perhaps the most important task along with the process of prioritization. Experience has shown that one can prescribe guidelines for structuring a hierarchy. Here are some suggestions for an elaborate design of a hierarchy:

(1) Identify the overall goal. What are you trying to accomplish? What is the main question?

(2) Identify the subgoals of the overall goal. If relevant, identify time horizons that affect the decision.

(3) Identify criteria that must be satisfied to fulfill the subgoals of the overall goal.

(4) Identify subcriteria under each criterion. Note that criteria or subcriteria may be specified in terms of ranges of values of parameters or in terms of verbal intensities such as high, medium, low.

(5) Identify the actors involved.

(6) Identify the actors' goals.

(7) Identify the actors' policies.

(8) Identify the people affected by the decision.

(9) Identify the objectives of these people.

(10) Identify options or outcomes to take that serve people's objectives best.

(11) For restricted yes-no decisions, take the most preferred outcome and compare the benefits and costs of making the decision with those of not making it.

(12) Do a benefit/cost analysis using marginal values and total priority values. Because we deal with dominance hierarchies, ask which alternative yields the greatest benefit; for costs: which alternative costs the most, and for risks, which alternative is more risky. Rate the top ranked alternative for each of the BOCR with respect to strategic criteria and subcriteria that are compared to obtain their priorities. Normalize the derived weights and use them to weight the BOCR. In the marginal case for each alternative divide the weighted benefit multiplied by the weighted opportunity and divide by the weighted cost times the weighted risk. Note whether the outcome is more or less than one. It is generally better to have the result more than one for the best alternative but may not always be necessary as is the case of having a major airline in a poor country. To obtain the total priority values, for each alternative add the weighted benefits and opportunities and subtract the weighted costs and risks. The outcome in this case may be negative. The final outcome from the marginal and the total results may not be the 
same. In general the total is what is needed, but the marginal is also valuable for actions with immediate rather than long term results.

(13) Perform sensitivity analysis on the outcome to determine its stability to changes in the judgments. If desired, include a criterion in each hierarchy called "other" or "the unknown" for which appropriate priority values may be derived from paired comparisons. Sensitivity testing with respect to such a criterion can determine the impact of the unknown on the outcome to the best of an experienced person's understanding. It must be understood that such a factor cannot be included to cover up for total ignorance about a decision. Only the wise should use it.

\section{AN EXAMPLE: The Hospice Problem}

Westmoreland County Hospital in Western Pennsylvania, like hospitals in many other counties around the nation, has been concerned with the costs of the facilities and manpower involved in taking care of terminally ill patients. Normally these patients do not need as much medical attention as do other patients. Those who best utilize the limited resources in a hospital are patients who require the medical attention of its specialists and advanced technology equipment, whose utilization depends on the demand of patients admitted into the hospital. The terminally ill need medical attention only episodically. Most of the time such patients need psychological support. Such support is best given by the patient's family, whose members are able to supply the love and care the patients most need. For the mental health of the patient, home therapy is a benefit. Most patients need the help of medical professionals only during a crisis. Some will also need equipment and surgery.

The planning association of the hospital wanted to develop alternatives and to choose the best one considering various criteria from the standpoint of the patient, the hospital, the community, and society at large.

In this problem, we need to consider the costs and benefits of the decision. Cost includes economic costs and all sorts of intangibles, such as inconvenience and pain. Such disbenefits are not directly related to benefits as their mathematical inverses, because patients infinitely prefer the benefits of good health to these intangible disbenefits. To study the problem, one needs to deal with benefits and with costs separately.

To keep matters simple we give an example of a decision made by considering benefits and costs only. No opportunities and risks were included as one usually must do in a more complex decision. The first author met with representatives of the planning association for several hours to decide on the best alternative. To make a decision by considering benefits and costs, one must first answer the question: In this problem, do the benefits justify the costs? If they do, then either the benefits are so much more important than the costs that the decision is based simply on benefits, or the two are so close in value that both the benefits and the costs should be considered. Then we use two hierarchies for the purpose and make the choice by forming the ratio from them of the (benefits priority/cost priority) for each alternative. One asks which is most beneficial in the benefits hierarchy of Figure 3 and which is most costly in the costs hierarchy of Figure 4.

If the benefits do not justify the costs, the costs alone determine the best alternative, that which is the least costly. In this example, we decided 


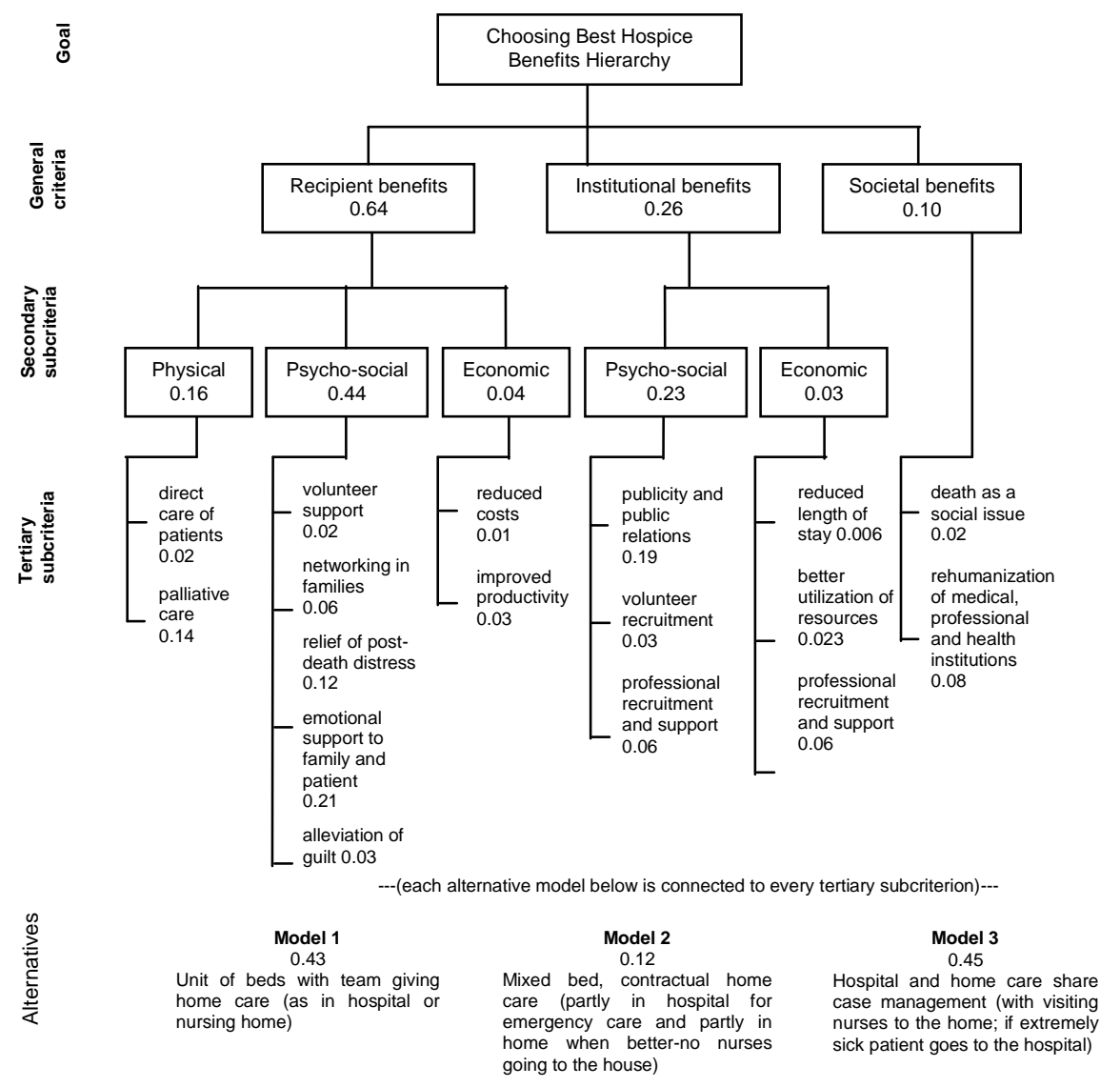

Figure 3 Benefits Hierarchy to Choose the Best Hospice Plan 


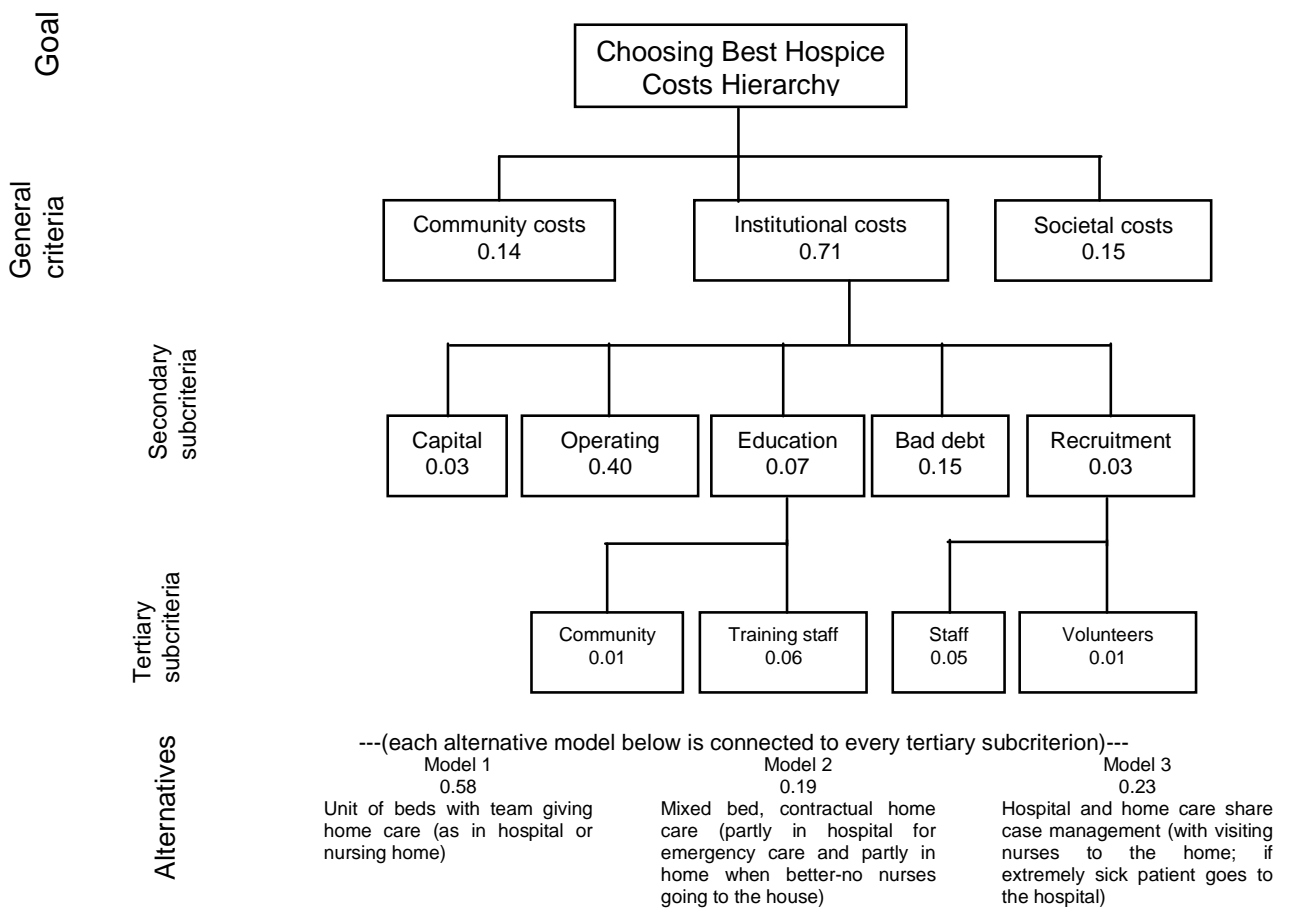

Figure 4 Costs Hierarchy to Choose the Best Hospice Plan

that both benefits and costs had to be considered in separate hierarchies. In a risk problem, a third hierarchy is used to determine the most desired alternative with respect to all three: benefits, costs, and risks. In this problem, we assumed risk to be the same for all contingencies. Whereas for most decisions one uses only a single hierarchy, we constructed two hierarchies for the hospice problem, one for benefits or gains (which model of hospice care yields the greater benefit) and one for costs or pains (which model costs more).

The planning association thought the concepts of benefits and costs were too general to enable it to make a decision. Thus, the planners further subdivided each (benefits and costs) into detailed subcriteria to enable the group to develop alternatives and to evaluate the finer distinctions the members perceived between the three alternatives. The alternatives were to care for terminally ill patients at the hospital, at home, or partly at the hospital and partly at home.

For each of the two hierarchies, benefits and costs, the goal clearly had to be choosing the best hospice. We placed this goal at the top of each hierarchy. Then the group discussed and identified overall criteria for each hierarchy; these criteria need not be the same for the benefits as for the costs.

The two hierarchies are fairly clear and straightforward in their description. They descend from the more general criteria in the second level to secondary subcriteria in the third level and then to tertiary subcriteria in the fourth level on to the alternatives at the bottom or fifth level.

At the general criteria level, each of the hierarchies, benefits or costs, involved three major interests. The decision should benefit the recipient, the institution, and society as a whole and their relative importance is the prime determinant as to which outcome is more likely to be preferred. We located these three elements on the second level of the benefits hierarchy. As the 
decision would benefit each party differently and the importance of the benefits to each recipient affects the outcome, the group thought that it was important to specify the types of benefit for the recipient and the institution. Recipients want physical, psycho-social and economic benefits, while the institution wants only psychosocial and economic benefits. We located these benefits in the third level of the hierarchy. Each of these in turn needed further decomposition into specific items in terms of which the decision alternatives could be evaluated. For example, while the recipient measures economic benefits in terms of reduced costs and improved productivity, the institution needed the more specific measurements of reduced length of stay, better utilization of resources, and increased financial support from the community. There was no reason to decompose the societal benefits into third level subcriteria and hence societal benefits connects directly to the fourth level. The group considered three models for the decision alternatives, and they are at the bottom (or fifth level in this case) of the hierarchy: in Model 1, the hospital provided full care to the patients; in Model 2, the family cares for the patient at home, and the hospital provides only emergency treatment (no nurses go to the house); and in Model 3, the hospital and the home share patient care (with visiting nurses going to the home).

In the costs hierarchy there were also three major interests in the second level that would incur costs or pains: community, institution, and society. In this decision the costs incurred by the patient were not included as a separate factor. Patient and family could be thought of as part of the community. We thought decomposition was necessary only for institutional costs. We included five such costs in the third level: capital costs, operating costs, education costs, bad debt costs, and recruitment costs. Educational costs apply to educating the community and training the staff. Recruitment costs apply to staff and volunteers. Since both the costs hierarchy and the benefits hierarchy concern the same decision, they both have the same alternatives in their bottom levels, even though the costs hierarchy has fewer levels.

The question now is how to use pairwise comparison judgments and derive priorities and synthesize them to obtain the overall benefits and costs of each of the three alternatives, and then again for the costs and combine the two outcomes into a single overall outcome. To do that we need to first explain in simple terms how the process of prioritization is carried out.

\section{Judgments and Comparisons}

A judgment is an expression of an opinion. A comparison is an expression of an opinion about the dominance (importance, preference or likelihood) of one thing over another. Dominance represents the intensity of strength. It is done every day through verbal expression that has some quantitative significance that we need to use to combine the many dominance judgments involved in a decision. The set of all such judgments in making comparisons with respect to a single property or goal can be represented in a square matrix in which the set of elements is compared with itself. It is a way of organizing all the judgments with respect to that property to be processed and synthesized along with other matrices of comparison judgments involved in that decision. Each judgment represents the dominance of an element in the column on the left of the matrix over an element in the row on top. It reflects the answers to two questions: which of the two elements is more important with respect to a higher level criterion, and how strongly.

As usual with the AHP, in both the cost and the benefits models, we compared the criteria and subcriteria according to their relative importance with respect to the parent element in the adjacent upper level. For example, the entries in the matrix shown in Table 9 are responses to the question: which general criterion is more important with respect to choosing the best hospice 
alternative and how strongly? Here recipient benefits are moderately more important than institutional benefits and are assigned the absolute number 3 in the $(1,2)$ or first-row secondcolumn position. Three signifies three times more. The reciprocal value is automatically entered in the $(2,1)$ position, where institutional benefits on the left are compared with recipient benefits at the top. Similarly a 5, corresponding to strong dominance or importance, is assigned to recipient benefits over social benefits in the $(1,3)$ position, and a 3 , corresponding to moderate dominance, is assigned to institutional benefits over social benefits in the $(2,3)$ position with corresponding reciprocals in the transpose positions of the matrix.

Judgments in a matrix may not be consistent. In eliciting judgments, one makes redundant comparisons to improve the validity of the answer, given that respondents may be uncertain or may make poor judgments in comparing some of the elements. Redundancy gives rise to multiple comparisons of an element with other elements and hence to numerical inconsistencies.

Table 9 Judgment Matrix for the Criteria of the Benefits Hierarchy

\begin{tabular}{|l|c|c|c|c|}
\hline $\begin{array}{c}\text { Choosing } \\
\text { best hospice }\end{array}$ & $\begin{array}{l}\text { Recipient } \\
\text { benefits }\end{array}$ & $\begin{array}{l}\text { Institutional } \\
\text { benefits }\end{array}$ & $\begin{array}{l}\text { Social } \\
\text { benefits }\end{array}$ & Priorities \\
\hline $\begin{array}{l}\text { Recipient } \\
\text { benefits }\end{array}$ & 1 & 3 & 5 & .64 \\
$\begin{array}{l}\text { Institutional } \\
\text { benefits } \\
\text { Social } \\
\text { benefits }\end{array}$ & $1 / 3$ & 1 & 3 & .26 \\
\hline
\end{tabular}

C.R. $=.033$

For example, where we compare recipient benefits with institutional benefits and with societal benefits, we have the respective judgments 3 and 5. Now if $x=3 y$ and $x=5 z$ then $3 y=5 z$ or $y=$ $5 / 3 \mathrm{z}$. If the judges were consistent, institutional benefits would be assigned the value $5 / 3$ instead of the 3 given in the matrix. Thus the judgments are inconsistent. In fact, we are not sure which judgments are the accurate ones and which are the cause of the inconsistency.

The process is repeated in all the matrices by asking the appropriate dominance or importance question. For example, the entries in the judgment matrix shown in Table 10 are responses to the question: which subcriterion yields the greater benefit with respect to institutional benefits and how strongly?

Here psycho-social benefits are regarded as very strongly more important than economic benefits, and 7 is entered in the $(1,2)$ position and $1 / 7$ in the $(2,1)$ position. 
Table 10 Judgment Matrix of Subcriteria with Respect to Institutional Benefits

\begin{tabular}{|l|c|c|c|}
\hline $\begin{array}{l}\text { Institutional } \\
\text { benefits }\end{array}$ & $\begin{array}{l}\text { Psycho- } \\
\text { social }\end{array}$ & Economic & Priorities \\
\hline $\begin{array}{l}\text { Psycho- } \\
\text { social }\end{array}$ & 1 & 7 & .875 \\
Economic & $1 / 7$ & 1 & .125 \\
\hline
\end{tabular}

In comparing the three models for patient care, we asked members of the planning association which model they preferred with respect to each of the covering or parent secondary criterion in level 3 or with respect to the tertiary criteria in level 4 . For example, for the subcriterion direct care (located on the left-most branch in the benefits hierarchy), we obtained a matrix of paired comparisons in Table 11 in which Model 1 is preferred over Models 2 and 3 by 5 and 3 respectively and Model 3 is preferred by 3 over Model 2. The group first made all the comparisons using semantic terms for the fundamental scale and then translated them to the corresponding numbers.

Table 11 Relative Benefits of the Models with Respect to Direct Care of Patients

\begin{tabular}{|c|c|c|c|c|}
\hline $\begin{array}{l}\text { Direct care of } \\
\text { patient }\end{array}$ & Model I & Model II & Model III & Priorities \\
\hline $\begin{array}{l}\text { Model I } \\
\text { unit team }\end{array}$ & 1 & 5 & 3 & .64 \\
\hline $\begin{array}{l}\text { Model II } \\
\text { mixed/home care }\end{array}$ & $1 / 5$ & 1 & $1 / 3$ & .10 \\
\hline $\begin{array}{l}\text { Model III } \\
\text { case management }\end{array}$ & $1 / 3$ & 3 & 1 & .26 \\
\hline
\end{tabular}

For the costs hierarchy, we again illustrate with three matrices. First the group compared the three major cost criteria and provided judgments in response to the question: which criterion is a more important determinant of the cost of a hospice model? Table 12 shows the judgments obtained.

Table 12 Judgment Matrix for the Criteria of the Costs Hierarchy

\begin{tabular}{|l|c|c|c|c|}
\hline $\begin{array}{l}\text { Choosing best } \\
\text { hospice (costs) }\end{array}$ & Community & Institutional & Societal & Priorities \\
\hline Community costs & 1 & $1 / 5$ & 1 & .14 \\
Institutional costs & 5 & 1 & 5 & .71 \\
Societal costs & 1 & $1 / 5$ & 1 & .14 \\
\hline
\end{tabular}

C.R. $=.000$ 
The group then compared the subcriteria under institutional costs and obtained the importance matrix shown in Table 13. The entries are responses to the question: which criterion incurs greater institutional costs and how strongly?

Finally we compared the three models to find out which incurs the highest cost for each criterion or subcriterion. Table 14 shows the results of comparing them with respect to the costs of recruiting staff.

Table 13 Judgment Matrix of Subcriteria Under Institutional Costs

\begin{tabular}{|l|c|c|c|c|c|c|}
\hline $\begin{array}{l}\text { Institutional } \\
\text { costs }\end{array}$ & Capital & Operating & Education & Bad debt & Recruitment & Priorities \\
\hline Capital & 1 & $1 / 7$ & $1 / 4$ & $1 / 7$ & 1 & .05 \\
Operating & 7 & 1 & 9 & 4 & 5 & .57 \\
Education & 4 & $1 / 9$ & 1 & $1 / 2$ & 1 & .01 \\
Bad debt & 7 & $1 / 4$ & 2 & 1 & 3 & .21 \\
Recruitment & 1 & $1 / 5$ & 1 & $1 / 3$ & 1 & .07 \\
\hline
\end{tabular}

Table 14 Relative Costs of the Models with Respect to Recruiting Staff

\begin{tabular}{|l|c|c|c|c|}
\hline $\begin{array}{l}\text { Institutional } \\
\text { costs for } \\
\text { recruiting staff }\end{array}$ & Model I & Model II & Model III & Priorities \\
\hline $\begin{array}{l}\text { Model I } \\
\text { unit team }\end{array}$ & 1 & 5 & 3 & .64 \\
$\begin{array}{l}\text { Model II } \\
\text { mixed/home care } \\
\text { Model III } \\
\text { case management }\end{array}$ & $1 / 5$ & 1 & $1 / 3$ & .10 \\
\hline
\end{tabular}

C.R. $=.08$

As shown in Table 15, we divided the benefits priorities by the costs priorities for each alternative to obtain the best alternative, model 3 , the one with the largest value for the ratio.

Table 15 shows two ways or modes of synthesizing the local priorities of the alternatives using the global priorities of their parent criteria: The distributive mode and the ideal mode. In the distributive mode, the weights of the alternatives sum to one. It is used when there is dependence among the alternatives and a unit priority is distributed among them. The ideal mode is used to obtain the single best alternative regardless of what other alternatives there are. In the ideal mode, the local priorities of the alternatives are divided by the largest value among them. This is done for each criterion; for each criterion one alternative becomes an ideal with value one. In both modes, the local priorities are weighted by the global priorities of the parent criteria and synthesized and the benefit-to-cost ratios formed. In this case, both modes lead to the same outcome for hospice, which is model 3. As we shall see below, we need both modes to deal with the effect of adding (or deleting) alternatives on an already ranked set. 
Table 15 Global and Ideal Modes of Synthesizing the Local Priorities of the Alternatives

\begin{tabular}{|c|c|c|c|c|c|c|c|}
\hline & & \multicolumn{3}{|c|}{ Distributive Mode } & \multicolumn{3}{|c|}{ Ideal Mode } \\
\hline Benefits & Priorities & $\begin{array}{l}\text { Model } \\
1\end{array}$ & $\begin{array}{l}\text { Model } \\
2\end{array}$ & $\begin{array}{l}T_{\text {Model }} \\
I_{3}\end{array}$ & $\begin{array}{l}\text { Model } \\
1\end{array}$ & $\begin{array}{l}\text { I Model } \\
\text { I } 2\end{array}$ & $\begin{array}{l}\text { I Model } \\
\text { I } 3\end{array}$ \\
\hline $\begin{array}{l}\text { Direct Care of Patient } \\
\text { Palliative Care } \\
\text { Volunteer Support } \\
\text { Networking in Families } \\
\text { Relief of Post Death Stress } \\
\text { Emotional Support of Family and Patient } \\
\text { Alleviation of Guilt } \\
\text { Reduced Economic Costs for Patient } \\
\text { Improved Productivity } \\
\text { Publicity and Public Relations } \\
\text { Volunteer Recruitment } \\
\text { Professional Recruitment and Support } \\
\text { Reduced Length of Stay } \\
\text { Better Utilization of Resources } \\
\text { Increased Monetary Support } \\
\text { Death as a Social Issue } \\
\text { Rehumanization of Institutions }\end{array}$ & $\begin{array}{l}.02 \\
.14 \\
.02 \\
.06 \\
.12 \\
.21 \\
.03 \\
.01 \\
.03 \\
.19 \\
.03 \\
.06 \\
.006 \\
.023 \\
.001 \\
.02 \\
.08\end{array}$ & $\begin{array}{l}0.64 \\
0.64 \\
0.09 \\
0.46 \\
0.30 \\
0.30 \\
0.30 \\
0.12 \\
0.12 \\
0.63 \\
0.64 \\
0.65 \\
0.26 \\
0.09 \\
0.73 \\
0.20 \\
0.24 \\
\end{array}$ & $\begin{array}{l}0.10 \\
0.10 \\
0.17 \\
0.22 \\
0.08 \\
0.08 \\
0.08 \\
0.65 \\
0.27 \\
0.08 \\
0.10 \\
0.23 \\
0.10 \\
0.22 \\
0.08 \\
0.20 \\
\end{array}$ & $\begin{array}{l}10.26 \\
10.26 \\
10.74 \\
10.32 \\
10.62 \\
10.62 \\
10.62 \\
10.23 \\
10.61 \\
10.29 \\
10.26 \\
10.12 \\
10.64 \\
10.69 \\
10.19 \\
10.60 \\
10.62 \\
\end{array}$ & $\begin{array}{l}1.000 \\
1.000 \\
0.122 \\
1.000 \\
0.484 \\
0.484 \\
0.484 \\
0.185 \\
0.197 \\
1.000 \\
1.000 \\
1.000 \\
0.406 \\
0.130 \\
1.000 \\
0.333 \\
0.387 \\
\end{array}$ & 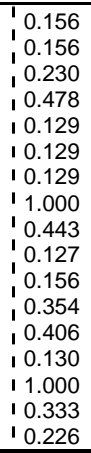 & 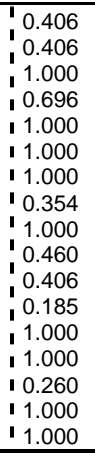 \\
\hline Synthesis & & 0.428 & 0.121 & 0.451 & 0.424 & 0.123 & 0.453 \\
\hline $\begin{array}{l}\text { Costs } \\
\text { Community Costs } \\
\text { Institutional Capital Costs } \\
\text { Institutional Operating Costs } \\
\text { Institutional Costs for Educating the Community } \\
\text { Institutional Costs for Training Staff } \\
\text { Institutional Bad Debt } \\
\text { Institutional Costs of Recruiting Staff } \\
\text { Institutional Costs of Recruiting Volunteers } \\
\text { Societal Costs }\end{array}$ & $\begin{array}{l}.14 \\
.03 \\
.40 \\
.01 \\
.06 \\
.15 \\
.05 \\
.01 \\
.15\end{array}$ & $\begin{array}{l}0.33 \\
0.76 \\
0.73 \\
0.65 \\
0.56 \\
0.60 \\
0.66 \\
0.60 \\
0.33\end{array}$ & $\begin{array}{ll} & \\
1 & 0.33 \\
1 & 0.09 \\
0.08 \\
0.24 \\
0.32 \\
0.20 \\
0.17 \\
0.20 \\
0.33\end{array}$ & $\begin{array}{ll} & \\
1 & 0.33 \\
1 & 0.15 \\
1 & 0.19 \\
1 & 0.11 \\
1 & 0.12 \\
1 & 0.20 \\
1 & 0.17 \\
1 & 0.20 \\
1 & 0.33 \\
\end{array}$ & $\begin{array}{l}1.000 \\
1.000 \\
1.000 \\
1.000 \\
1.000 \\
1.000 \\
1.000 \\
1.000 \\
1.000\end{array}$ & $\begin{array}{l}\text { I } 1.000 \\
10.118 \\
10.110 \\
10.369 \\
10.571 \\
10.333 \\
10.258 \\
10.333 \\
11.000\end{array}$ & 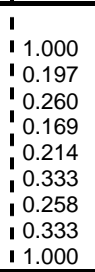 \\
\hline Synthesis & & 0.583 & 0.192 & 0.224 & 0.523 & 0.229 & 0.249 \\
\hline Benefit/Cost Ratio & & 0.734 & 0.630 & 2.013 & 0.811 & 0.537 & 1.819 \\
\hline
\end{tabular}

Model 3 has the largest ratio of benefits to costs in both the distributive and ideal modes, and the hospital selected it for treating terminal patients. This need not always be the case. In this case, there is dependence of the personnel resources allocated to the three models because some of these resources would be shifted based on the decision. Therefore the distributive mode is the appropriate method of synthesis. If the alternatives were sufficiently distinct with no dependence in their definition, the ideal mode would be the way to synthesize.

We also performed marginal analysis to determine where the hospital should allocate additional resources for the greatest marginal return. To perform marginal analysis, we first ordered the alternatives by increasing cost priorities and then formed the benefit-to-cost ratios corresponding to the smallest cost, followed by the ratios of the differences of successive benefits to costs. If this difference in benefits is negative, the new alternative is dropped from consideration and the process continued. The alternative with the largest marginal ratio is then chosen. For the costs and corresponding benefits from the synthesis rows in Table 15 we obtained:

$$
\begin{array}{llll}
\text { Costs: } & 0.20 & 0.21 & 0.59 \\
\text { Benefits: } & 0.12 & 0.45 & 0.43
\end{array}
$$

$\frac{.12}{.20}=0.60 \quad \frac{.45-.12}{.21-.20}=33 \quad \frac{.43-.45}{.59-.21}=-0.051$

From these values we compute the marginal ratios as the final priorities: The third alternative is not a contender for resources because its marginal return is negative. The second alternative is 
best. In fact, in addition to adopting the third model, the hospital management chose the second model of hospice care for further development.

\section{ABSOLUTE MEASUREMENT - Rating Alternatives One at a Time}

People are able to make two kinds of comparisons - absolute and relative. In absolute comparisons, people compare alternatives with a standard in their memory that they have developed through experience. In relative comparisons, they compared alternatives in pairs according to a common attribute, as we did throughout the hospice example.

People use absolute measurement (sometimes also called rating) to rank independent alternatives one at a time in terms of rating intensities for each of the criteria. An intensity is a range of variation of a criterion that enables one to distinguish the quality of an alternative for that criterion. An intensity may be expressed as a numerical range of values if the criterion is measurable or defined in qualitative terms.

For example, if ranking students is the objective and one of the criteria on which they are to be ranked is performance in mathematics, the mathematics ratings might be: excellent, good, average, below average, poor; or, using the usual school terminology, A, B, C, D, and F. Relative comparisons are first used to set priorities on the ratings themselves. If desired, one can fit a continuous curve through the derived intensities. This concept may go against our socialization. However, it is perfectly reasonable to ask how much an $\mathrm{A}$ is preferred to a $\mathrm{B}$ or to a $\mathrm{C}$. The judgment of how much an $\mathrm{A}$ is preferred to a $\mathrm{B}$ might be different under different criteria. Perhaps for mathematics an $\mathrm{A}$ is very strongly preferred to a B, while for physical education an $\mathrm{A}$ is only moderately preferred to a B. So the end result might be that the ratings are scaled differently. For example one could have the scale values for the ratings as shown in Table 16:

Table 16 Examples of Scale Values for Ratings

\begin{tabular}{|l|c|c|}
\hline & Math & $\begin{array}{l}\text { Physical } \\
\text { Education }\end{array}$ \\
\hline A & 0.50 & 0.30 \\
B & 0.30 & 0.30 \\
C & 0.15 & 0.20 \\
D & 0.04 & 0.10 \\
E & 0.01 & 0.10 \\
\hline
\end{tabular}

The alternatives are then rated or ticked off one at a time using the intensities. We will illustrate absolute measurement with an example. A firm evaluates its employees for raises. The criteria are dependability, education, experience, and quality. Each criterion is subdivided into intensities, standards, or subcriteria (Figure 5). The managers set priorities for the criteria by comparing them in pairs. They then pairwise compare the intensities according to priority with respect to their parent criterion (as in Table 17) or with respect to a subcriterion if they are using a deeper hierarchy. The priorities of the intensities are divided by the largest intensity for each criterion (second column of priorities in Figure 5). 


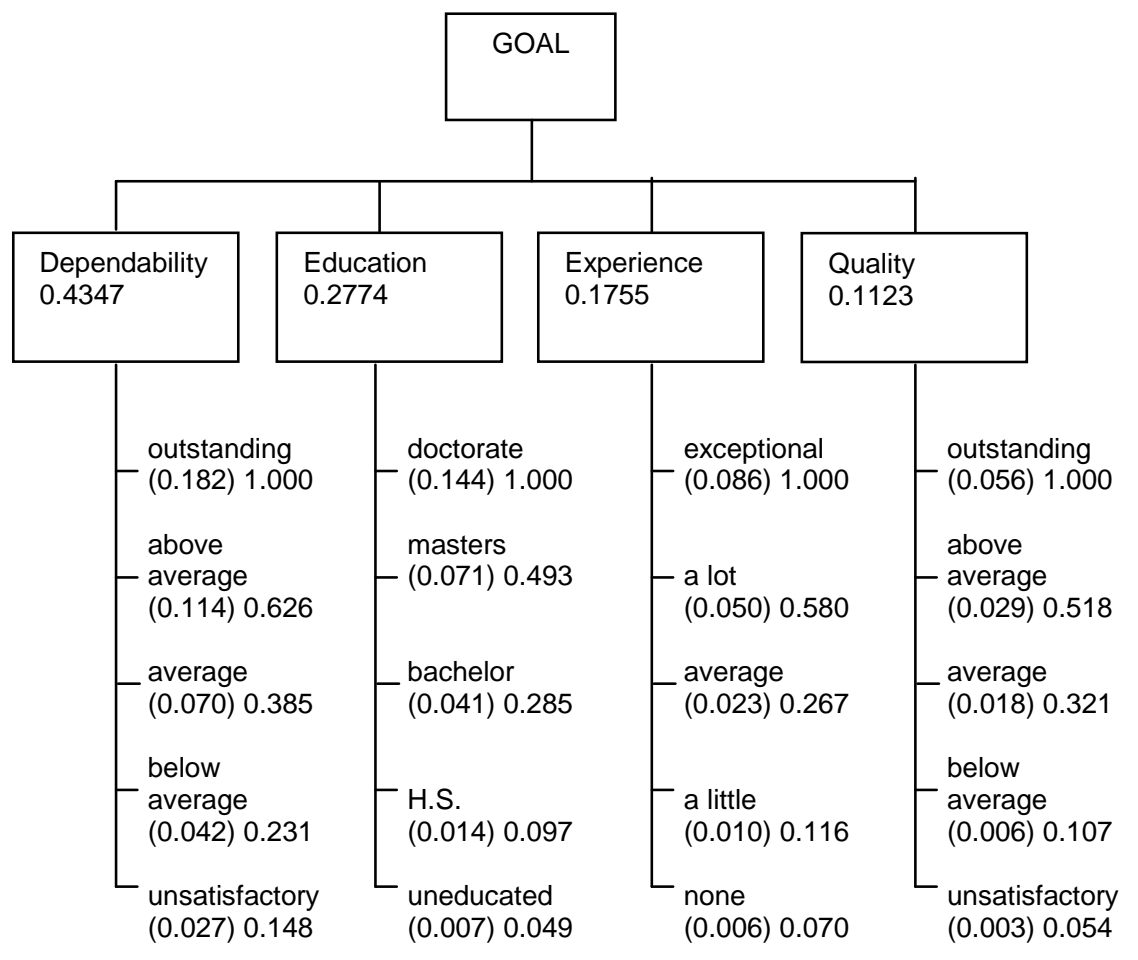

Figure 5 Hierarchy with Absolute Measurement

Table 17 shows a paired comparison matrix of intensities with respect to dependability. The managers answer the question: which intensity is more important and by how much with respect to dependability. The priorities of the intensities for each criterion are divided by the largest one and multiplied by the priority of the criterion. Finally the managers rate each individual (Table 18) by assigning the intensity rating that applies to him or her under each criterion. The scores of these intensities are each weighted by the priority of its criterion and summed to derive a total ratio scale score for the individual (shown on the right of Table 18). These numbers belong to an absolute scale, and the managers can give salary increases precisely in proportion to the ratios of these numbers. Adams gets the highest score and Kesselman the lowest. This approach can be used whenever it is possible to set priorities for intensities of criteria; people can usually do this when they have sufficient experience with a given operation. This normative mode requires that alternatives be rated one by one without regard to how many there may be and how high or low any of them rates on prior standards. Some corporations have insisted that they no longer trust the normative standards of their experts and that they prefer to make paired comparisons of their alternatives. Still, when there is wide agreement on standards, the absolute mode saves time in rating a large number of alternatives. 
Table 17 Ranking Intensities: Which intensity is preferred most with respect to dependability and how strongly?

\begin{tabular}{|l|l|l|l|l|l|l|l|}
\hline $\begin{array}{l}\text { Depend- } \\
\text { ability }\end{array}$ & $\begin{array}{l}\text { Outstan } \\
\text { ding }\end{array}$ & $\begin{array}{l}\text { Above } \\
\text { Average }\end{array}$ & Average & $\begin{array}{l}\text { Below } \\
\text { Average }\end{array}$ & $\begin{array}{l}\text { Unsatis- } \\
\text { factory }\end{array}$ & $\begin{array}{l}\text { Priorit } \\
\text { ies }\end{array}$ & $\begin{array}{l}\text { Idealiz } \\
\text { ed } \\
\text { Priorit } \\
\text { ies }\end{array}$ \\
\hline Outstanding & 1.0 & 2.0 & 3.0 & 4.0 & 5.0 & 0.419 & 1.000 \\
Above Avg & $1 / 2$ & 1.0 & 2.0 & 3.0 & 4.0 & 0.263 & 0.628 \\
Average & $1 / 3$ & $1 / 2$ & 1.0 & 2.0 & 3.0 & 0.160 & 0.382 \\
Below Avg. & $1 / 4$ & $1 / 3$ & $1 / 2$ & 1.0 & 2.0 & 0.097 & 0.232 \\
Unsatisfactory & $1 / 5$ & $1 / 4$ & $1 / 3$ & $1 / 2$ & 1.0 & 0.062 & 0.148 \\
\hline
\end{tabular}

Table 18 Rating Alternatives

\begin{tabular}{|l|l|l|l|l|l|}
\hline Employees & $\begin{array}{l}\text { Dependabilit } \\
\text { y }\end{array}$ & $\begin{array}{l}\text { Education } \\
.2774\end{array}$ & $\begin{array}{l}\text { Experie } \\
\text { nce } \\
.4347\end{array}$ & $\begin{array}{l}\text { Quality } \\
.1123\end{array}$ & Total \\
\hline 1. Adams, V & Outstanding & Bachelor & A Little & Outstandin & 0.646 \\
2. Becker, L & Average & Bachelor & A Little & Outstandin & 0.379 \\
3. Hayat, F & Average & Masters & A Lot & Below & 0.418 \\
4. Kessel, S & Above & H.S. & None & Average & 0.369 \\
5. O'Shea, K & Average & Doctorate & A Lot & Above & 0.605 \\
6. Peters, T & Average & Doctorate & A Lot & Average & 0.583 \\
7. Tobias, K & Above & Bachelor & Averag & Above & 0.456 \\
\hline
\end{tabular}

\section{ON THE ADMISSION OF CHINA TO THE WORLD TRADE ORGANIZATION (WTO) [7]}

This section was taken from an analysis done in 2000 carried out before the US Congress acted favorably on China joining the WTO and was hand-delivered to many of the members of the committee including its Chairperson. Since 1986, China had been attempting to join the multilateral trade system, the General Agreement on Tariffs and Trade (GATT) and, its successor, the World Trade Organization (WTO). According to the rules of the 135-member nations of WTO, a candidate member must reach a trade agreement with any existing member country that wishes to trade with it. By the time this analysis was done, China signed bilateral agreements with 30 countries - including the US (November 1999) - out of 37 members that had requested a trade deal with it [5].

As part of its negotiation deal with the US, China asked the US to remove its annual review of China's Normal Trade Relations (NTR) status, until 1998 called Most Favored Nation (MFN) status. In March 2000, President Clinton sent a bill to Congress requesting a Permanent Normal Trade Relations (PNTR) status for China. The analysis was done and copies sent to leaders and some members in both houses of Congress before the House of Representatives voted on the bill, May 24, 2000. The decision by the US Congress on China's trade-relations status will have an influence on US interests, in both direct and indirect ways. Direct impacts include changes in 
economic, security and political relations between the two countries as the trade deal is actualized. Indirect impacts will occur when China becomes a WTO member and adheres to WTO rules and principles. China has said that it would join the WTO only if the US gives it Permanent Normal Trade Relations status.

It is likely that Congress will consider four options the least likely is that the US will deny China both PNTR and annual extension of NTR status. The other three options are:

1. Passage of a clean PNTR bill: Congress grants China Permanent Normal Trade Relations status with no conditions attached. This option would allow implementation of the November 1999 WTO trade deal between China and the Clinton administration. China would also carry out other WTO principles and trade conditions.

2. Amendment of the current NTR status bill: This option would give China the same trade position as other countries and disassociate trade from other issues. As a supplement, a separate bill may be enacted to address other matters, such as human rights, labor rights, and environmental issues.

3. Annual Extension of NTR status: Congress extends China's Normal Trade Relations status for one more year, and, thus, maintains the status quo.

The conclusion of the study is that the best alternative is granting China PNTR status. China now has that status.

Our analysis involves four steps. First, we prioritize the criteria in each of the benefits, costs, opportunities and risks hierarchies with respect to the goal. Figure 6 shows the resulting prioritization of these criteria. The alternatives and their priorities are shown under each criterion both in the distributive and in the ideal modes. The ideal priorities of the alternatives were used appropriately to synthesize their final values beneath each hierarchy.

The priorities shown in Figure 6 were derived from judgments that compared the elements involved in pairs. For readers to estimate the original pairwise judgments (not shown here) one forms the ratio of the corresponding two priorities shown, leave them as they are, or take the closest whole number, or its reciprocal if it is less than 1.0.

The idealized values are shown in parentheses after the original distributive priorities obtained from the eigenvector. The ideal values are obtained by dividing each of the distributive priorities by the largest one among them. For the Costs and Risks structures, the question is framed as to which is the most costly or risky alternative. That is, the most costly alternative ends up with the highest priority.

It is likely that, in a particular decision, the benefits, costs, opportunities and risks (BOCR) are not equally important, so we must also prioritize them. This is shown in Table 19. The priorities for the economic, security and political factors themselves were established as shown in Figure 7 and used to rate the importance of the top ideal alternative for each of the benefits, costs, opportunities and risks from Table 19. Finally, we used the priorities of the latter to combine the synthesized priorities of the alternatives in the four hierarchies, using the normalized reciprocal - priorities of the alternatives under costs and risks, to obtain their final ranking, as shown in Table 20. 


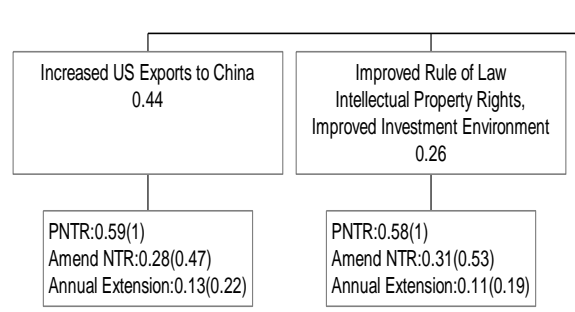

Benefits to US $(0.25)$

Benefits Synthesis (Ideal): PNTR 1.00 Amend NTR 0.51 Annual Extension 0.21

\begin{tabular}{|c|c|c|}
\hline & 1 & 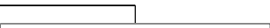 \\
\hline $\begin{array}{c}\text { China's Promise to Respect } \\
\text { Anti-Dumping and } \\
\text { Section } 201 \text { Provisions } \\
0.18\end{array}$ & $\begin{array}{l}\text { Increased Employment in US } \\
0.07\end{array}$ & $\begin{array}{l}\text { Benefits to Lower Income Consumers } \\
0.05\end{array}$ \\
\hline $\begin{array}{l}\text { PNTR:0.65(1) } \\
\text { Amend NTR:0.23(0.53) } \\
\text { Annual Extension:0.12(0.19) }\end{array}$ & $\begin{array}{l}\text { PNTR:0.54(1) } \\
\text { Amend NTR:0.30(0.55) } \\
\text { Amend NTR:0.30(0.30) }\end{array}$ & \begin{tabular}{|l|} 
PNTR:0.58(1) \\
Amend NTR:0.31(0.53) \\
Annual Extension:0.11(0.19)
\end{tabular} \\
\hline
\end{tabular}

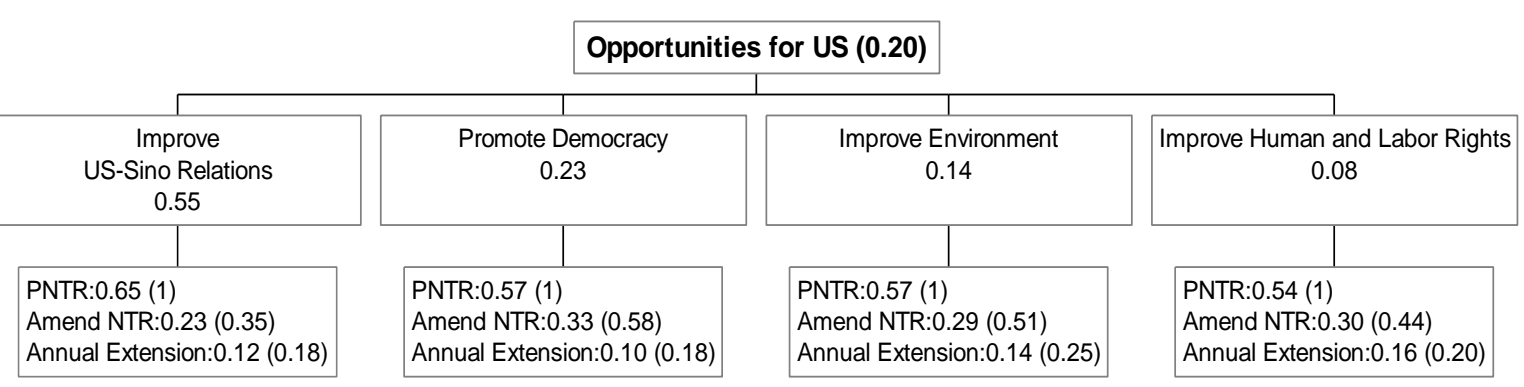

Opportunities Synthesis (Ideal): PNTR 1 Amend NTR 0.43 Annual Extension 0.13

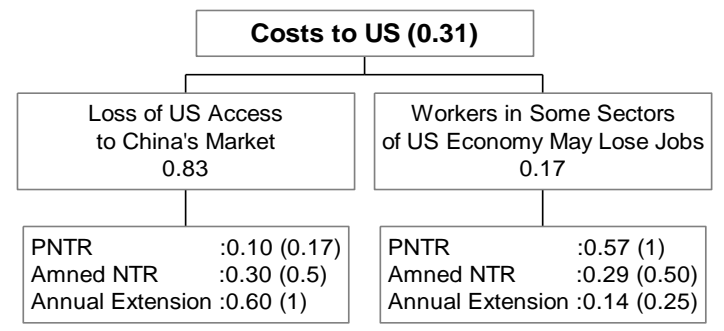

Costs Synthesis (which is more costly, Ideal): PNTR 0.31 Amend NTR 0.50 Annual Extension 0.87

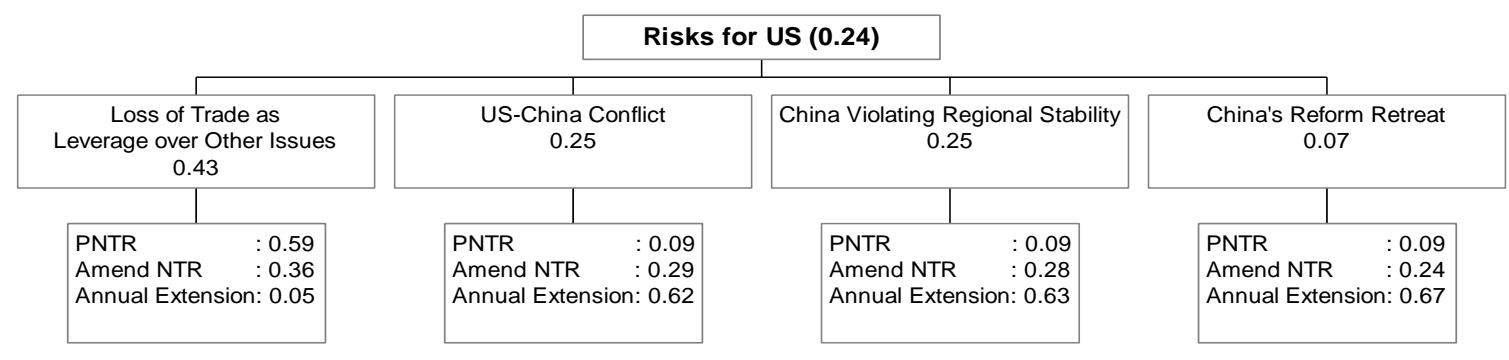

Risks Synthesis (more risky, Ideal): PNTR 0.54 Amend NTR 0.53 Annual Extension 0.58

Figure 6 Hierarchies for Rating Benefits, Costs, Opportunities, and Risks 


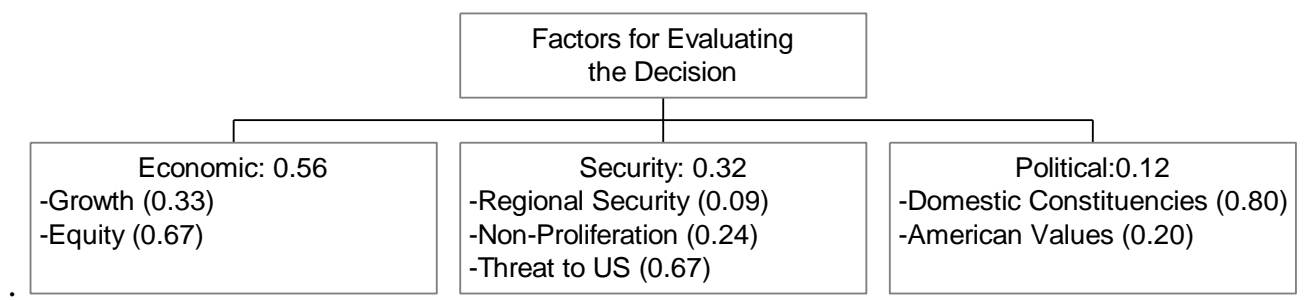

Figure 7 Prioritizing the Strategic Criteria to be used in Rating the BOCR

How to derive the priority shown next to the goal of each of the four hierarchies shown in Figure 7 is outlined in Table 19. We rated each of the four merits: benefits, costs, opportunities and risks of the dominant PNTR alternative, as it happens to be in this case, in terms of intensities for each assessment criterion. The intensities, Very High, High, Medium, Low, and Very Low were themselves prioritized in the usual pairwise comparison matrix to determine their priorities. We then assigned the appropriate intensity for each merit on all assessment criteria. The outcome is as found in the bottom row of 19 .

Table 19 Priority Ratings for the Merits: Benefits, Costs, Opportunities, and Risks Intensities: Very High (0.42), High (0.26), Medium (0.16), Low (0.1), Very Low (0.06)

\begin{tabular}{|c|l|c|c|c|c|}
\hline & & Benefits & Opportunities & Costs & Risks \\
\hline $\begin{array}{c}\text { Economic } \\
(0.56)\end{array}$ & Growth (0.19) & High & Medium & Very Low & Very Low \\
\cline { 2 - 6 } & Equity $(0.37)$ & Medium & Low & High & Low \\
\hline \multirow{3}{*}{$\begin{array}{c}\text { Security } \\
(0.32)\end{array}$} & Regional $(0.03)$ & Low & Medium & Medium & High \\
\cline { 2 - 6 } & $\begin{array}{l}\text { Non-Proliferation } \\
(0.08)\end{array}$ & Medium & High & Medium & High \\
\cline { 2 - 6 } & Threat to US (0.21) & High & High & Very High & Very High \\
\hline $\begin{array}{c}\text { Political } \\
(0.12)\end{array}$ & Constituencies $(0.1)$ & High & Medium & Very High & High \\
\cline { 2 - 6 } & $\begin{array}{l}\text { American Values } \\
(0.02)\end{array}$ & Very Low & Low & Low & Medium \\
\hline Priorities & & 0.25 & 0.20 & 0.31 & 0.24 \\
\hline
\end{tabular}

We are now able to obtain the overall priorities of the three major decision alternatives listed earlier, given as columns in Table 20 which gives two ways of synthesis for the ideal mode. We see in bold that PNTR is the dominant alternative any way we synthesize as in the last four columns. 
Table 20 Two Methods of Synthesizing BOCR Using the Ideal Mode

\begin{tabular}{|c|c|c|c|c|c|c|}
\hline 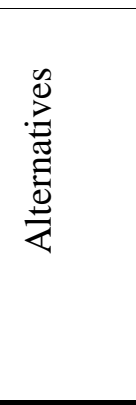 & $\begin{array}{l}\stackrel{0}{0} \\
\stackrel{0}{0} \\
\infty \\
(0.25)\end{array}$ & 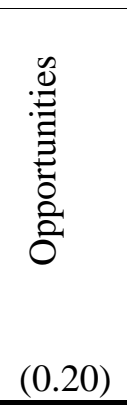 & $\begin{array}{l}\tilde{0}_{0}^{\infty} \\
0 \\
(0.31)\end{array}$ & $\begin{array}{l}\frac{n}{n} \\
\frac{n}{2} \\
(0.24)\end{array}$ & 仓ئ & $\begin{array}{l}a \\
1 \\
1 \\
0 \\
1 \\
0 \\
0 \\
+ \\
0\end{array}$ \\
\hline PNTR & 1 & 1 & 0.31 & 0.54 & 4 & 0.22 \\
\hline $\begin{array}{l}\text { Amend } \\
\text { NTR }\end{array}$ & 0.51 & 0.43 & 0.50 & 0.53 & 0.51 & -0.07 \\
\hline $\begin{array}{c}\text { Annual } \\
\text { Exten. }\end{array}$ & 0.21 & 0.13 & 0.87 & 0.58 & 0.05 & -0.31 \\
\hline
\end{tabular}

In general one can weight the $\mathrm{B}, \mathrm{O}, \mathrm{C}$ and $\mathrm{R}$ values by the corresponding $\mathrm{b}, \mathrm{o}, \mathrm{c}$ and $\mathrm{r}$ in the $\mathrm{BO} / \mathrm{CR}$ formula to determine if it is advantageous to implement the best alternative with a value greater or less than one.

We have laid the basic foundation with hierarchies for what we need to deal with networks involving interdependencies. Let us now turn to that subject.

\section{NETWORKS, DEPENDENCE AND FEEDBACK $[2,3]$}

In Figure 8, we exhibit a hierarchy and a network. A hierarchy is comprised of a goal, levels of elements and connections between the elements. These connections are oriented only to elements in lower levels. A hierarchy is authoritarian. It passes the word down from higher up. It describes our commitments, what is important to us and what we prefer even if we imagine it all. A hierarchy is a special case of a network. In a hierarchy connections go only in one direction. In the view of a hierarchy such as that shown in Figure the levels correspond to clusters in a network. A network has clusters of elements, with the elements in one cluster being connected to elements in another cluster (outer dependence) or the same cluster (inner dependence). A network is concerned with all the influences from people and from nature that can affect an outcome. It is a model of continual change because everything affects everything else and what we do now can change the importance of the criteria that control the evolution of the outcome.

There are two kinds of influence: outer and inner. In the first one compares the influence of elements in a cluster on elements in another cluster with respect to a control criterion. In inner influence one compares the influence of elements in a group on each one. For example if one takes a family of father mother and child, and then take them one at a time say the child first, one asks who contributes more to the child's survival, its father or its mother, itself or its father, itself or its mother. In this case the child is not so important in contributing to its survival as its parents are. But if we take the mother and ask the same question on who contributes to her survival more, herself or her husband, herself would be higher, or herself and the child, again herself. Another example of inner dependence is making electricity. To make electricity you need steel to make turbines, and you need fuel. So we have the electric industry, the steel industry and the fuel 
industry. What does the electric industry depend on more to make electricity, itself or the steel industry? Steel is more important; itself or the fuel industry? The fuel industry is much more important; the steel or fuel industry? Fuel is more important. The electric industry does not need its own electricity to make electricity. It needs fuel. Its electricity is only used to light the rooms, which it may not even need.

If we think about it carefully everything can be seen to influence everything else including itself according to many criteria. The world is more interdependent than we know how to deal with using our ways of thinking and taking action. The ANP is our logical way to deal with dependence.
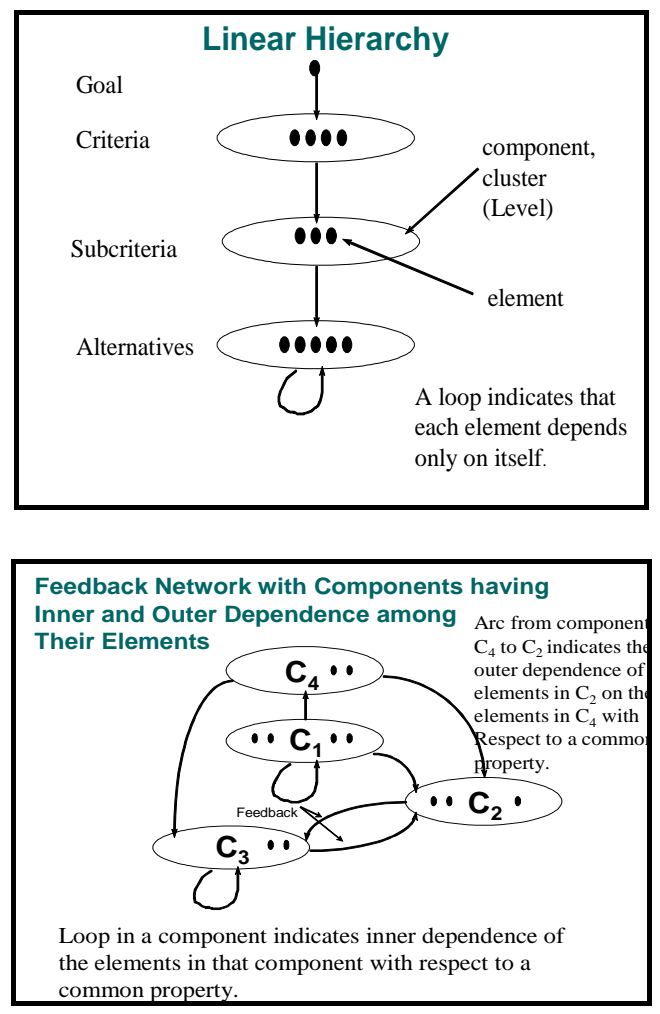

\section{Figure 8 How a Hierarchy Compares to a Network}

The priorities derived from pairwise comparison matrices are entered as parts of the columns of a supermatrix. The supermatrix represents the influence priority of an element on the left of the matrix on an element at the top of the matrix with respect to a particular control criterion. A supermatrix along with an example of one of its general entry matrices is shown in Figure 8. The component $\mathrm{C}_{1}$ in the supermatrix includes all the priority vectors derived for nodes that are "parent" nodes in the $\mathrm{C}_{1}$ cluster. Figure 9 gives the supermatrix of a hierarchy and Figure 10 shows the $k$ th power of that supermatrix which is the same as hierarchic composition in the $(\mathrm{k}, 1)$ position. 


\section{The Supermatrix of a Network}

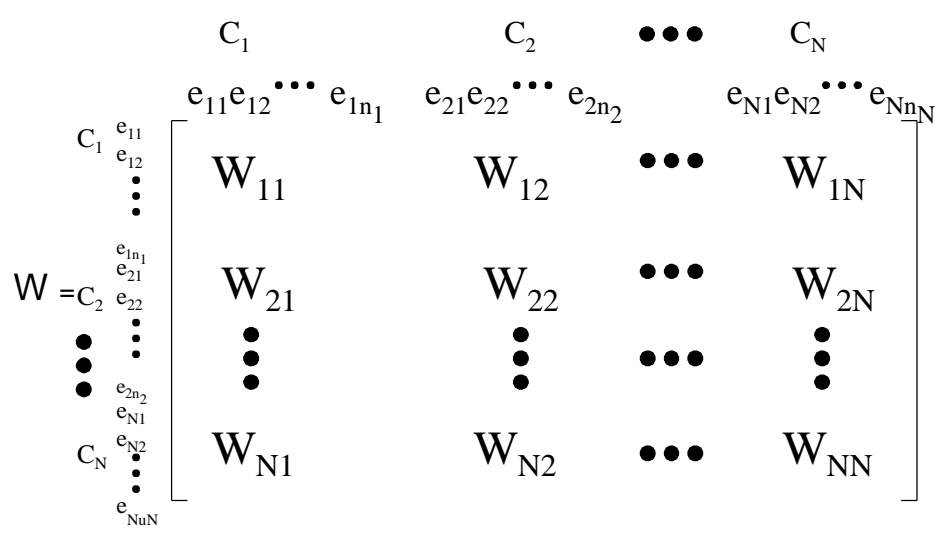

\section{$\mathrm{W}_{\mathrm{ij}}$ Component of Supermatrix}

$\mathrm{W}_{\mathrm{ij}}=\left[\begin{array}{cccc}\mathrm{W}_{\mathrm{i} 1}^{\left(\mathrm{j}_{1}\right)} & \mathrm{W}_{\mathrm{i} 1}^{\left(\mathrm{j}_{2}\right)} & \cdots & \mathrm{W}_{\mathrm{i} 1}^{\left(\mathrm{jn}_{\mathrm{j}}\right)} \\ \mathrm{W}_{\mathrm{i} 2}^{\left(\mathrm{j}_{1}\right)} & \mathrm{W}_{\mathrm{i} 2}^{\left(\mathrm{j}_{2}\right)} & \bullet & \mathrm{W}_{\mathrm{i} 2}^{\left(\mathrm{jn}_{\mathrm{j}}\right)} \\ \vdots & \vdots & & \vdots \\ \mathrm{W}_{\mathrm{in}}^{\left(\mathrm{j}_{1}\right)} & \mathrm{W}_{\mathrm{in}_{\mathrm{i}}}^{\left(\mathrm{j}_{2}\right)} & \bullet & \mathrm{W}_{\mathrm{in}}^{\left(\mathrm{jn}_{\mathrm{j}}\right)}\end{array}\right]$

Figure 9 The Supermatrix of a Network and Detail of a Component in it 


\section{Supermatrix of a Hierarchy}

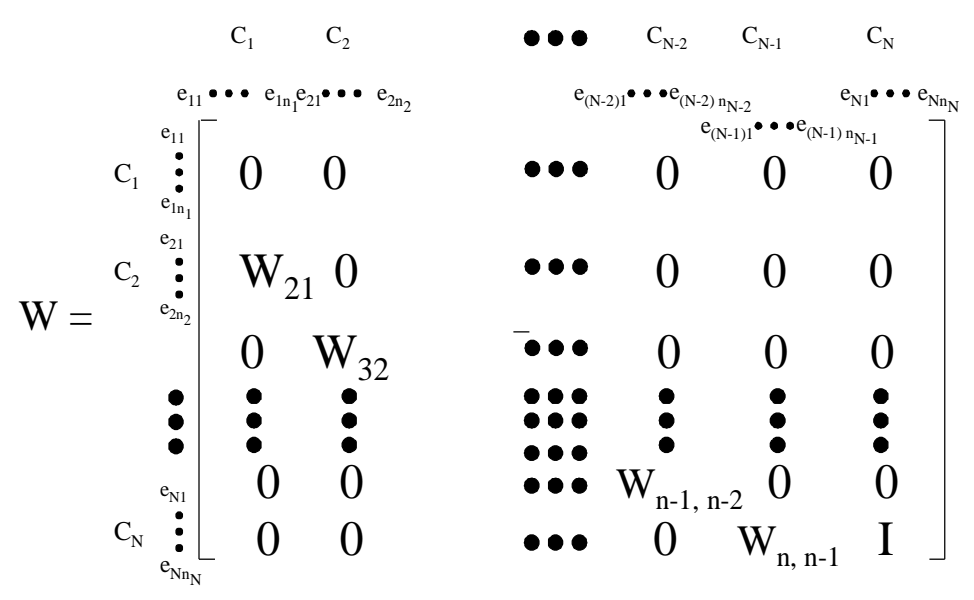

Figure 8 The Supermatrix of a Hierarchy

$$
W^{k}=\left[\begin{array}{cccccc}
0 & 0 & \ldots & 0 & 0 & 0 \\
0 & 0 & \ldots & 0 & 0 & 0 \\
\vdots & \vdots & \vdots: \vdots & \vdots & \vdots & \vdots \\
0 & 0 & \ldots & 0 & 0 & 0 \\
W_{n, n-1} W_{n-1, n-2} \ldots W_{32} W_{21} & W_{n, n-1} W_{n-1, n-2} \ldots W_{32} & \ldots W_{n, n-1} W_{n-1, n-2} & W_{n, n-1} & I
\end{array}\right]
$$

\section{Figure 10 The Limit Supermatrix of a Hierarchy (Corresponds to Hierarchical Composition)}

The $(n, 1)$ entry of the limit supermatrix of a hierarchy as shown in Figure 10 above gives the hierarchic composition principle.

In the ANP we look for steady state priorities from a limit super matrix. To obtain the limit we must raise the matrix to powers. Each power of the matrix captures all transitivities of an order that is equal to that power. The limit of these powers, according to Cesaro Summability, is equal to the limit of the average sum of all the powers of the matrix. All order transitivities are captured by this series of powers of the matrix. The outcome of the ANP is nonlinear and rather complex. The limit may not converge unless the matrix is column stochastic that is each of its columns sums to one. If the columns sum to one then from the fact that the principal eigenvalue of a matrix lies between its largest and smallest column sums, we know that the principal eigenvalue of a stochastic matrix is equal to one.

But for the supermatrix we already know that $\lambda_{\max }(T)=1$ which follows from: 


$$
\begin{aligned}
& \max \sum_{j=1}^{n} a_{i j} \geq \sum_{j=1}^{n} a_{i j} \frac{w_{j}}{w_{i}}=\lambda_{\max } \text { for } \max w_{i} \\
& \min \sum_{j=1}^{n} a_{i j} \leq \sum_{j=1}^{n} a_{i j} \frac{w_{j}}{w_{i}}=\lambda_{\max } \text { for } \min w_{i}
\end{aligned}
$$

Thus for a row stochastic matrix we have

$$
1=\min \sum_{j=1}^{n} a_{i j} \leq \lambda_{\max } \leq \max \sum_{j=1}^{n} a_{i j}=1
$$

The same type of argument applies to a matrix that is column stochastic.

Now we know, for example, from a theorem due to J.J. Sylvester [2] that when the eigenvalues of a matrix $W$ are distinct that an entire function $f(x)$ (power series expansion of $f(x)$ converges for all finite values of $x$ ) with $x$ replaced by $W$, is given by

$$
f(W)=\sum_{i=1}^{n} f\left(\lambda_{i}\right) Z\left(\lambda_{i}\right), Z\left(\lambda_{i}\right)=\frac{\prod_{j \neq i}\left(\lambda_{j} I-A\right)}{\prod_{j \neq i}\left(\lambda_{j}-\lambda_{i}\right)}, \sum_{i=1}^{n} Z\left(\lambda_{i}\right)=I, Z\left(\lambda_{i}\right) Z\left(\lambda_{j}\right)=0, Z^{2}\left(\lambda_{i}\right)=Z\left(\lambda_{i}\right)
$$

where I and 0 are the identity and the null matrices respectively.

A similar expression is also available when some or all of the eigenvalues have multiplicities. We can see that if, as we need in our case, $f(W)=W^{k}$, then $f\left(\lambda_{i}\right)=\lambda_{i}^{k}$ and as $k \rightarrow \infty$ the only terms that give a finite nonzero value are those for which the modulus of $\lambda_{i}$ is equal to one. The fact that $W$ is stochastic ensures this because its largest eigenvalue is equal to one. The priorities of the alternatives (or any set of elements in a component) are obtained by normalizing the corresponding values in the appropriate columns of the limit matrix. When $W$ has zeros and is reducible (its graph is not strongly connected so there is no path from some point to some other point) the limit can cycle and a Cesaro average over the different limits of the cycle is taken. For a more complete treatment, see the book by Saaty on the ANP.

\section{ANP Formulation of the Classic AHP School Example}

We show in Figure 11 below the hierarchy for choosing a best school, and in the corresponding supermatrix, and its limit supermatrix in Figure 12 the priorities of three schools involved in a decision to choose the best one. They are precisely what one obtains by hierarchic composition using the AHP. The priorities of the criteria with respect to the goal and those of the alternatives with respect to each criterion are clearly discernible in the supermatrix itself. Note that there is an identity submatrix for the alternatives with respect to the alternatives in the lower right hand part of the matrix. The level of alternatives in a hierarchy is a sink cluster of nodes that absorbs priorities but does not pass them on. This calls for using an identity submatrix for them in the supermatrix. 


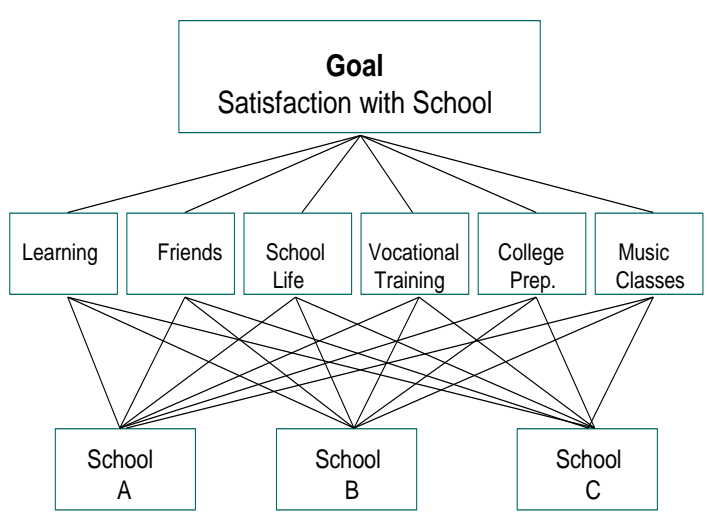

Figure 11 The School Choice Hierarchy

\section{The School Hierarchy as Supermatrix}

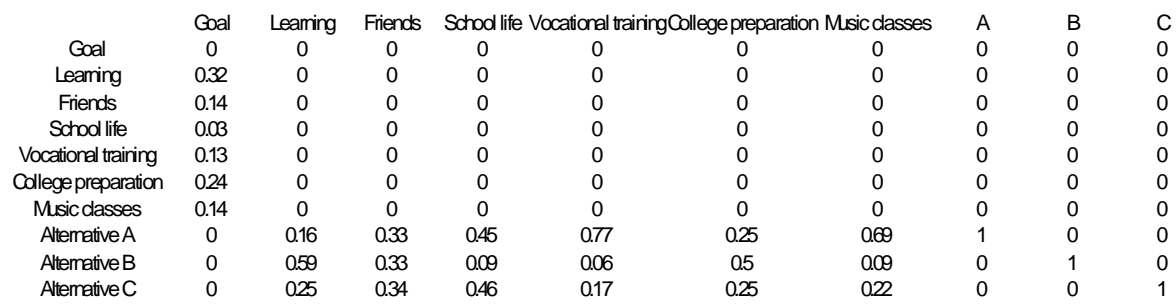

\section{Limiting Supermatrix \& Hierarchic Composition}

\begin{tabular}{|c|c|c|c|c|c|c|c|c|c|}
\hline & Goal & Leaming & Friends & School life & Vocational trair & ngCollege preparation & Music dasses & A & B \\
\hline Goal & 0 & 0 & 0 & 0 & 0 & 0 & 0 & 0 & 0 \\
\hline Leaming & 0 & 0 & 0 & 0 & 0 & 0 & 0 & 0 & 0 \\
\hline Fiends & 0 & 0 & 0 & 0 & 0 & 0 & 0 & 0 & 0 \\
\hline School life & 0 & 0 & 0 & 0 & 0 & 0 & 0 & 0 & 0 \\
\hline Vocational training & 0 & 0 & 0 & 0 & 0 & 0 & 0 & 0 & 0 \\
\hline College preparation & 0 & 0 & 0 & 0 & 0 & 0 & 0 & 0 & 0 \\
\hline Misic dasses & 0 & 0 & 0 & 0 & 0 & 0 & 0 & 0 & 0 \\
\hline Attemative A & 0.3676 & 0.16 & 0.33 & 0.45 & 0.77 & 0.25 & 0.69 & 1 & 0 \\
\hline Altemative B & 0.3781 & 0.59 & 0.33 & 0.09 & 0.06 & 0.5 & 0.09 & 0 & 1 \\
\hline AltemativeC & 0.2543 & 025 & 0.34 & 0.46 & 0.17 & 0.25 & 0.22 & 0 & 0 \\
\hline
\end{tabular}

Figure 12 The Limit Supermatrix of the School Choice Hierarchy shows same Result as Hierarchic Composition

9. Market Share Examples Mainly to Justify WiTH EXisting Measurements SUbJECTIVE JUdGMENT THAT DOES NOT REFER TO ANY NUMERICAL DATA

An ANP Network with a Single Control Criterion - Market Share 
A market share estimation model is structured as a network of clusters and nodes. The object is to try to determine the relative market share of competitors in a particular business, or endeavor, by considering what affects market share in that business and introduce them as clusters, nodes and influence links in a network. The decision alternatives are the competitors and the synthesized results are their relative dominance. The relative dominance results can then be compared against some outside measure such as dollars. If dollar income is the measure being used, the incomes of the competitors must be normalized to get it in terms of relative market share.

The clusters might include customers, service, economics, advertising, and the quality of goods. The customers' cluster might then include nodes for the age groups of the people that buy from the business: teenagers, 20-33 year olds, 34-55 year olds, 55-70 year olds, and over 70. The advertising cluster might include newspapers, TV, Radio, and Fliers. After all the nodes are created one starts by picking a node and linking it to the other nodes in the model that influence it. The "children" nodes will then be pairwise compared with respect to that node as a "parent" node. An arrow will automatically appear going from the cluster the parent node cluster to the cluster with its children nodes. When a node is linked to nodes in its own cluster, the arrow becomes a loop on that cluster and we say there is inner dependence.

The linked nodes in a given cluster are pairwise compared for their influence on the node they are linked from (the parent node) to determine the priority of their influence on the parent node. Comparisons are made as to which is more important to the parent node in capturing "market share". These priorities are then entered in the supermatrix for the network.

The clusters are also pairwise compared to establish their importance with respect to each cluster they are linked from, and the resulting matrix of numbers is used to weight the corresponding blocks of the original unweighted supermatrix to obtain the weighted supermatrix. This matrix is then raised to powers until it converges to yield the limit supermatrix. The relative values for the companies are obtained from the columns of the limit supermatrix that in this case are all the same because the matrix is irreducible. Normalizing these numbers yields the relative market share.

If comparison data in terms of sales in dollars, or number of members, or some other known measures are available, one can use these relative values to validate the outcome. The AHP/ANP has a compatibility index to determine how close the ANP result is to the known measure. It involves taking the Hadamard product of the matrix of ratios of the ANP outcome and the transpose of the matrix of ratios of the actual outcome summing all the coefficients and dividing by $n^{2}$. The requirement is that the value should be close to 1 .

\section{Compatibility Index}

Let us show first that the priority vector $w=\left(w_{1}, \ldots, w_{n}\right)$ is completely compatible with itself. Thus we form the matrix of all possible ratios $W=\left(w_{i j}\right)=\left(w_{i} / w_{j}\right)$ from this vector. This matrix is reciprocal, that is $w_{j i}=1 / w_{i j}$. The Hadamard product of a reciprocal matrix $W$ and its transpose $W^{T}$ is given by:

$$
W o W^{T}=\left(\begin{array}{rrr}
w_{1} / w_{1} & \ldots & w_{1} / w_{n} \\
\vdots & & \vdots \\
w_{n} / w_{1} & \ldots & w_{n} / w_{n}
\end{array}\right) \circ\left(\begin{array}{rrr}
w_{1} / w_{1} & \ldots & w_{n} / w_{1} \\
\vdots & & \vdots \\
w_{1} / w_{n} & \ldots & w_{n} / w_{n}
\end{array}\right)=\left(\begin{array}{rrr}
1 & \ldots & 1 \\
\vdots & & \vdots \\
1 & \ldots & 1
\end{array}\right)=\left(\begin{array}{c}
1 \\
\vdots \\
1
\end{array}\right)(1 \cdots 1) \equiv e e^{T}
$$


The sum of the elements of a matrix $A$ can be written as $e^{T} A e$. In particular we have $e^{T} A o A^{T} e=n^{2}$ for the sum of the elements of the Hadamard product of a matrix and its transpose. The index of compatibility is the sum resulting from the Hadamard product divided by $n^{2}$. Thus a vector is completely compatible with itself as $\frac{n^{2}}{n^{2}}=1$. Now we have an idea of how to define a measure of compatibility for two matrices $A$ and $B$. It is given by $\frac{1}{n^{2}} e^{T} A o B^{T} e$. Note that a reciprocal matrix of judgments that is inconsistent is not itself a matrix of ratios from a given vector. However, such a matrix has a principal eigenvector and thus we speak of the compatibility of the matrix of judgments and the matrix formed from ratios of the principal eigenvector. We have the following theorem for a reciprocal matrix of judgments and the matrix $W$ of the ratios of its principal eigenvector:

Theorem: $\frac{1}{n^{2}} e^{T}$ Ao $W^{T} e=\frac{\lambda_{\max }}{n}$

Proof: From $A w=\lambda_{\max } w$ we have $\sum_{j=1}^{n} a_{i j} w_{j}=\lambda_{\max } w_{i}$

and $\quad \frac{1}{n^{2}} e^{T} A o W^{\mathrm{T}} e=\frac{1}{n^{2}} \sum_{i=1}^{n} \sum_{j=1}^{n} a_{i j} \frac{w_{j}}{w_{i}}=\frac{\lambda_{\max }}{n}$

We want this ratio to be close to one or in general not much more than 1.01 and be less than this value for small size matrices. It is in accord with the idea that a $10 \%$ deviation is at the upper end of acceptability.

We will give two examples of market share estimation showing details of the process in the first example and showing only the models and results in the second example.

\section{EXAMPLE 1: ESTIMATING THE RELATIVE MARKET SHARE OF WALMART, KMART AND TARGET}

The network for the ANP model shown in Figure 13 describes quite well the influences that determine the market share of these companies. We will not use space in this paper to describe the clusters and their nodes in greater detail. 


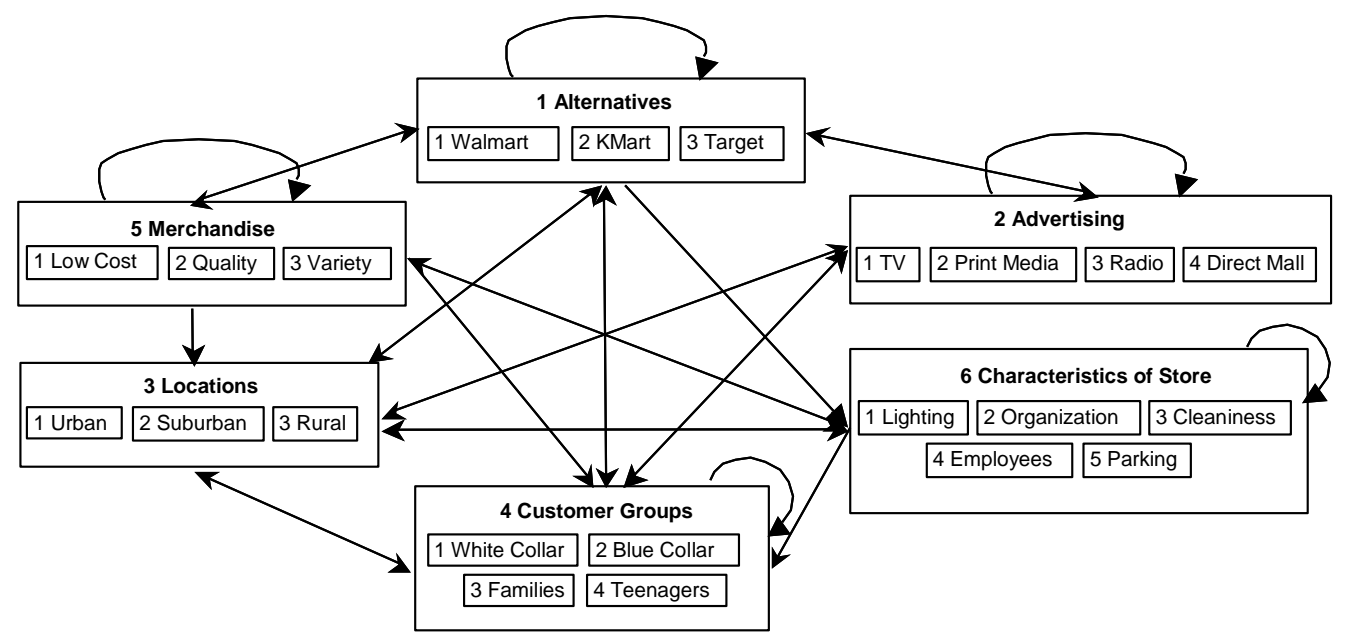

Figure 13 The Clusters and Nodes of a Model to Estimate the Relative Market Share of Walmart, Kmart and Target.

\section{THE UNWEIGHTED SUPERMATRIX}

The unweighted supermatrix is constructed from the priorities derived from the different pairwise comparisons. The nodes, grouped by the clusters they belong to, are the labels of the rows and columns of the supermatrix. The column for a node $a$ contains the priorities of the nodes that have been pairwise compared with respect to $a$. The supermatrix for the network in Figure 12 is shown in Table 21. 
Table 21 The Unweighted Supermatrix, Displayed in Two Parts

\begin{tabular}{|c|c|c|c|c|c|c|c|c|c|c|c|}
\hline & \multicolumn{3}{|c|}{1 Alternatives } & \multicolumn{3}{|c|}{2 Advertising } & & \multicolumn{3}{|c|}{3 Locations } \\
\hline & & 1 Walmart & 2 KMart & 3 Target & $1 \mathrm{TV}$ & 2 Print Media & 3 Radio & $\begin{array}{l}4 \text { Direct } \\
\text { Mail }\end{array}$ & 1 Urban & 2 Suburban & 3 Rura \\
\hline \multirow[t]{3}{*}{1 Alternatives } & 1 Walmart & 0.000 & 0.833 & 0.833 & 0.687 & 0.540 & 0.634 & 0.661 & 0.614 & 0.652 & 0.683 \\
\hline & 2 KMart & 0.750 & 0.000 & 0.167 & 0.186 & 0.297 & 0.174 & 0.208 & 0.268 & 0.235 & 0.200 \\
\hline & 3 Target & 0.250 & 0.167 & 0.000 & 0.127 & 0.163 & 0.192 & 0.131 & 0.117 & 0.113 & 0.117 \\
\hline \multirow[t]{4}{*}{2 Advertising } & $1 \mathrm{TV}$ & 0.553 & 0.176 & 0.188 & 0.000 & 0.000 & 0.000 & 0.000 & 0.288 & 0.543 & 0.558 \\
\hline & 2 Print Media & 0.202 & 0.349 & 0.428 & 0.750 & 0.000 & 0.800 & 0.000 & 0.381 & 0.231 & 0.175 \\
\hline & 3 Radio & 0.062 & 0.056 & 0.055 & 0.000 & 0.000 & 0.000 & 0.000 & 0.059 & 0.053 & 0.048 \\
\hline & 4 Direct Mail & 0.183 & 0.420 & 0.330 & 0.250 & 0.000 & 0.200 & 0.000 & 0.273 & 0.173 & 0.219 \\
\hline \multirow[t]{3}{*}{3 Locations } & 1 Urban & 0.114 & 0.084 & 0.086 & 0.443 & 0.126 & 0.080 & 0.099 & 0.000 & 0.000 & 0.000 \\
\hline & 2 Suburban & 0.405 & 0.444 & 0.628 & 0.387 & 0.416 & 0.609 & 0.537 & 0.000 & 0.000 & 0.000 \\
\hline & 3 Rural & 0.481 & 0.472 & 0.285 & 0.169 & 0.458 & 0.311 & 0.364 & 0.000 & 0.000 & 0.000 \\
\hline \multirow[t]{4}{*}{4 Cust.Groups } & 1 White Collar & 0.141 & 0.114 & 0.208 & 0.165 & 0.155 & 0.116 & 0.120 & 0.078 & 0.198 & 0.092 \\
\hline & 2 Blue Collar & 0.217 & 0.214 & 0.117 & 0.165 & 0.155 & 0.198 & 0.203 & 0.223 & 0.116 & 0.224 \\
\hline & 3 Families & 0.579 & 0.623 & 0.620 & 0.621 & 0.646 & 0.641 & 0.635 & 0.656 & 0.641 & 0.645 \\
\hline & 4 Teenagers & 0.063 & 0.049 & 0.055 & 0.048 & 0.043 & 0.045 & 0.041 & 0.043 & 0.045 & 0.038 \\
\hline \multirow[t]{3}{*}{5 Merchandise } & 1 Low Cost & 0.362 & 0.333 & 0.168 & 0.000 & 0.000 & 0.000 & 0.000 & 0.000 & 0.000 & 0.000 \\
\hline & 2 Quality & 0.261 & 0.140 & 0.484 & 0.000 & 0.000 & 0.000 & 0.000 & 0.000 & 0.000 & 0.000 \\
\hline & 3 Variety & 0.377 & 0.528 & 0.349 & 0.000 & 0.000 & 0.000 & 0.000 & 0.000 & 0.000 & 0.000 \\
\hline \multirow{5}{*}{6 Characteristic } & 1 Lighting & 0.000 & 0.000 & 0.000 & 0.000 & 0.000 & 0.000 & 0.000 & 0.000 & 0.000 & 0.000 \\
\hline & 2 Organization & 0.000 & 0.000 & 0.000 & 0.000 & 0.000 & 0.000 & 0.000 & 0.000 & 0.000 & 0.000 \\
\hline & 3 Cleanliness & 0.000 & 0.000 & 0.000 & 0.000 & 0.000 & 0.000 & 0.000 & 0.000 & 0.000 & 0.000 \\
\hline & 4 Employees & 0.000 & 0.000 & 0.000 & 0.000 & 0.000 & 0.000 & 0.000 & 0.000 & 0.000 & 0.000 \\
\hline & 5 Parking & 0.000 & 0.000 & 0.000 & 0.000 & 0.000 & 0.000 & 0.000 & 0.000 & 0.000 & 0.000 \\
\hline
\end{tabular}




\begin{tabular}{|c|c|c|c|c|c|c|c|c|c|c|c|c|c|}
\hline & \multicolumn{3}{|c|}{$\begin{array}{c} \\
\\
\text { Custom. } \\
\text { Groups } \\
\end{array}$} & & \multicolumn{3}{|c|}{$\begin{array}{l}5 \text { Merchan- } \\
\text { dise }\end{array}$} & \multicolumn{5}{|c|}{$\begin{array}{c}\text { 6Charac- } \\
\text { teristics } \\
\text { of Store } \\
\end{array}$} \\
\hline & & $\begin{array}{l}1 \text { White } \\
\text { Collar }\end{array}$ & $\begin{array}{l}2 \text { Blue } \\
\text { Collar }\end{array}$ & 3 Familie & 4 Teens & 1 Low Cost & 2 Quality & $\begin{array}{c}3 \\
\text { Variety }\end{array}$ & 1Light'ng & 2 Organiz. & 3 Clean & $\begin{array}{c}4 \\
\text { Emp- } \\
\text { loyees }\end{array}$ & 5 Park \\
\hline \multirow[t]{3}{*}{ 1Alternat. } & 1 Walmart & 0.637 & 0.661 & 0.630 & 0.691 & 0.661 & 0.614 & 0.648 & 0.667 & 0.655 & 0.570 & 0.644 & 0.558 \\
\hline & 2 KMart & 0.105 & 0.208 & 0.218 & 0.149 & 0.208 & 0.117 & 0.122 & 0.111 & 0.095 & 0.097 & 0.085 & 0.122 \\
\hline & 3 Target & 0.258 & 0.131 & 0.151 & 0.160 & 0.131 & 0.268 & 0.230 & 0.222 & 0.250 & 0.333 & 0.271 & 0.320 \\
\hline \multirow[t]{4}{*}{2 Advertis. } & $1 \mathrm{TV}$ & 0.323 & 0.510 & 0.508 & 0.634 & 0.000 & 0.000 & 0.000 & 0.000 & 0.000 & 0.000 & 0.000 & 0.000 \\
\hline & $\begin{array}{l}2 \text { Print } \\
\text { Med. }\end{array}$ & 0.214 & 0.221 & 0.270 & 0.170 & 0.000 & 0.000 & 0.000 & 0.000 & 0.000 & 0.000 & 0.000 & 0.000 \\
\hline & 3 Radio & 0.059 & 0.063 & 0.049 & 0.096 & 0.000 & 0.000 & 0.000 & 0.000 & 0.000 & 0.000 & 0.000 & 0.000 \\
\hline & $\begin{array}{l}\text { 4 Direct } \\
\text { Mail }\end{array}$ & 0.404 & 0.206 & 0.173 & 0.100 & 0.000 & 0.000 & 0.000 & 0.000 & 0.000 & 0.000 & 0.000 & 0.000 \\
\hline \multirow[t]{3}{*}{3 Locations } & 1 Urban & 0.167 & 0.094 & 0.096 & 0.109 & 0.268 & 0.105 & 0.094 & 0.100 & 0.091 & 0.091 & 0.111 & 0.067 \\
\hline & 2 Suburban & 0.833 & 0.280 & 0.308 & 0.309 & 0.117 & 0.605 & 0.627 & 0.433 & 0.455 & 0.455 & 0.444 & 0.293 \\
\hline & 3 Rural & 0.000 & 0.627 & 0.596 & 0.582 & 0.614 & 0.291 & 0.280 & 0.466 & 0.455 & 0.455 & 0.444 & 0.641 \\
\hline \multirow[t]{4}{*}{ 4Cust. Grps } & $\begin{array}{l}1 \text { White } \\
\text { Col. }\end{array}$ & 0.000 & 0.000 & 0.279 & 0.085 & 0.051 & 0.222 & 0.165 & 0.383 & 0.187 & 0.242 & 0.165 & 0.000 \\
\hline & $\begin{array}{l}2 \text { Blue } \\
\text { Collar }\end{array}$ & 0.000 & 0.000 & 0.649 & 0.177 & 0.112 & 0.159 & 0.165 & 0.383 & 0.187 & 0.208 & 0.165 & 0.000 \\
\hline & 3 Families & 0.857 & 0.857 & 0.000 & 0.737 & 0.618 & 0.566 & 0.621 & 0.185 & 0.583 & 0.494 & 0.621 & 0.000 \\
\hline & 4 Teenagers & 0.143 & 0.143 & 0.072 & 0.000 & 0.219 & 0.053 & 0.048 & 0.048 & 0.043 & 0.056 & 0.048 & 0.000 \\
\hline \multirow[t]{3}{*}{ 5Merchand. } & 1 Low Cost & 0.000 & 0.000 & 0.000 & 0.000 & 0.000 & 0.800 & 0.800 & 0.000 & 0.000 & 0.000 & 0.000 & 0.000 \\
\hline & 2 Quality & 0.000 & 0.000 & 0.000 & 0.000 & 0.750 & 0.000 & 0.200 & 0.000 & 0.000 & 0.000 & 0.000 & 0.000 \\
\hline & 3 Variety & 0.000 & 0.000 & 0.000 & 0.000 & 0.250 & 0.200 & 0.000 & 0.000 & 1.000 & 0.000 & 0.000 & 0.000 \\
\hline \multirow[t]{5}{*}{6 Character. } & 1 Lighting & 0.000 & 0.000 & 0.000 & 0.000 & 0.000 & 0.000 & 0.000 & 0.000 & 0.169 & 0.121 & 0.000 & 0.250 \\
\hline & 2 Organiz. & 0.000 & 0.000 & 0.000 & 0.000 & 0.000 & 0.000 & 0.000 & 0.251 & 0.000 & 0.575 & 0.200 & 0.750 \\
\hline & 3 Cleanli. & 0.000 & 0.000 & 0.000 & 0.000 & 0.000 & 0.000 & 0.000 & 0.673 & 0.469 & 0.000 & 0.800 & 0.000 \\
\hline & 4 Employee & 0.000 & 0.000 & 0.000 & 0.000 & 0.000 & 0.000 & 0.000 & 0.000 & 0.308 & 0.304 & 0.000 & 0.000 \\
\hline & 5 Parking & 0.000 & 0.000 & 0.000 & 0.000 & 0.000 & 0.000 & 0.000 & 0.075 & 0.055 & 0.000 & 0.000 & 0.000 \\
\hline
\end{tabular}

\section{The Cluster MATrix}

The cluster themselves must be compared to establish their relative importance and use it to weight the supermatrix to make it column stochastic. A cluster impacts another cluster when it is linked from it, that is, when at least one node in the source cluster is linked to nodes in the target cluster. The clusters linked from the source cluster are pairwise compared for the importance of their impact on it with respect to market share, resulting in the column of priorities for that cluster in the cluster matrix. The process is repeated for each cluster in the network to obtain the matrix shown in Table 22. An interpretation of the priorities in the first column is that Merchandise (0.442) and Locations (0.276) have the most impact on Alternatives, the three competitors.

Table 22 The Cluster Matrix

\begin{tabular}{|l|cccccc|}
\hline \multicolumn{2}{|c}{ 1 Alternatives } & 2 Advertising & 3 Locations & $\begin{array}{c}\text { 4 Customer } \\
\text { Groups }\end{array}$ & $\begin{array}{c}\mathbf{5} \\
\text { Merchandis } \\
\text { e }\end{array}$ & $\begin{array}{c}\mathbf{6} \\
\text { Characteristics } \\
\text { of Store }\end{array}$ \\
\hline 1 Alternatives & 0.137 & 0.174 & 0.094 & 0.057 & 0.049 & 0.037 \\
2 Advertising & 0.091 & 0.220 & 0.280 & 0.234 & 0.000 & 0.000 \\
$\begin{array}{l}\text { 3 Locations } \\
\text { 4 Customer } \\
\text { Groups }\end{array}$ & 0.276 & 0.176 & 0.000 & 0.169 & 0.102 & 0.112 \\
$\begin{array}{l}\text { 5 Merchandise } \\
\text { 6 Characteristics } \\
\text { of Store }\end{array}$ & 0.054 & 0.429 & 0.627 & 0.540 & 0.252 & 0.441 \\
\hline
\end{tabular}




\section{THE WEIGHTED SUPERMATRIX}

The weighted supermatrix shown in Table 23 is obtained by multiplying each entry in a block of the component at the top of the supermatrix by the priority of influence of the component on the left from the cluster matrix in Table 22. For example, the first entry, 0.137, in Table 22 is used to multiply each of the nine entries in the block (Alternatives, Alternatives) in the unweighted supermatrix shown in Table 21. This gives the entries for the (Alternatives, Alternatives) component in the weighted supermatrix of Table 23. Each column in the weighted supermatrix has a sum of 1 , and thus the matrix is stochastic and thus converges or is periodic.

Table 23 The Weighted Supermatrix (Given in two parts because it is too wide to display in one piece)

\begin{tabular}{|c|c|c|c|c|c|c|c|c|c|c|c|}
\hline & \multicolumn{3}{|c|}{1 Alternatives } & \multicolumn{3}{|c|}{2 Advertising } & & \multicolumn{3}{|c|}{3 Locations } \\
\hline & & 1 Walmart & 2 KMart & 3 Target & $1 \mathrm{TV}$ & 2 Print Media & 3 Radio & 4 Direct Mail & 1 Urban & 2 Suburban & 3 Rura \\
\hline \multirow[t]{3}{*}{1 Alternatives } & 1 Walmart & 0.000 & 0.114 & 0.114 & 0.120 & 0.121 & 0.110 & 0.148 & 0.058 & 0.061 & 0.064 \\
\hline & 2 KMart & 0.103 & 0.000 & 0.023 & 0.033 & 0.066 & 0.030 & 0.047 & 0.025 & 0.022 & 0.019 \\
\hline & 3 Target & 0.034 & 0.023 & 0.000 & 0.022 & 0.037 & 0.033 & 0.029 & 0.011 & 0.011 & 0.011 \\
\hline \multirow[t]{4}{*}{2 Advertising } & $1 \mathrm{TV}$ & 0.050 & 0.016 & 0.017 & 0.000 & 0.000 & 0.000 & 0.000 & 0.080 & 0.152 & 0.156 \\
\hline & 2 Print Media & 0.018 & 0.032 & 0.039 & 0.165 & 0.000 & 0.176 & 0.000 & 0.106 & 0.064 & 0.049 \\
\hline & 3 Radio & 0.006 & 0.005 & 0.005 & 0.000 & 0.000 & 0.000 & 0.000 & 0.016 & 0.015 & 0.014 \\
\hline & 4 Direct Mail & 0.017 & 0.038 & 0.030 & 0.055 & 0.000 & 0.044 & 0.000 & 0.076 & 0.048 & 0.061 \\
\hline \multirow[t]{3}{*}{3 Locations } & 1 Urban & 0.031 & 0.023 & 0.024 & 0.078 & 0.028 & 0.014 & 0.022 & 0.000 & 0.000 & 0.000 \\
\hline & 2 Suburban & 0.112 & 0.123 & 0.174 & 0.068 & 0.094 & 0.107 & 0.121 & 0.000 & 0.000 & 0.000 \\
\hline & 3 Rural & 0.133 & 0.130 & 0.079 & 0.030 & 0.103 & 0.055 & 0.082 & 0.000 & 0.000 & 0.000 \\
\hline \multirow[t]{4}{*}{4 Cust.Groups } & $\begin{array}{l}1 \text { White } \\
\text { Collar }\end{array}$ & 0.008 & 0.006 & 0.011 & 0.071 & 0.086 & 0.050 & 0.066 & 0.049 & 0.124 & 0.058 \\
\hline & 2 Blue Collar & 0.012 & 0.011 & 0.006 & 0.071 & 0.086 & 0.085 & 0.112 & 0.140 & 0.073 & 0.141 \\
\hline & 3 Families & 0.031 & 0.033 & 0.033 & 0.267 & 0.356 & 0.275 & 0.350 & 0.411 & 0.402 & 0.404 \\
\hline & 4 Teenagers & 0.003 & 0.003 & 0.003 & 0.021 & 0.024 & 0.019 & 0.023 & 0.027 & 0.028 & 0.024 \\
\hline \multirow[t]{3}{*}{5 Merchandise } & 1 Low Cost & 0.160 & 0.147 & 0.074 & 0.000 & 0.000 & 0.000 & 0.000 & 0.000 & 0.000 & 0.000 \\
\hline & 2 Quality & 0.115 & 0.062 & 0.214 & 0.000 & 0.000 & 0.000 & 0.000 & 0.000 & 0.000 & 0.000 \\
\hline & 3 Variety & 0.166 & 0.233 & 0.154 & 0.000 & 0.000 & 0.000 & 0.000 & 0.000 & 0.000 & 0.000 \\
\hline \multirow[t]{5}{*}{6 Characteristic } & 1 Lighting & 0.000 & 0.000 & 0.000 & 0.000 & 0.000 & 0.000 & 0.000 & 0.000 & 0.000 & 0.000 \\
\hline & 2 Organization & 0.000 & 0.000 & 0.000 & 0.000 & 0.000 & 0.000 & 0.000 & 0.000 & 0.000 & 0.000 \\
\hline & 3 Cleanliness & 0.000 & 0.000 & 0.000 & 0.000 & 0.000 & 0.000 & 0.000 & 0.000 & 0.000 & 0.000 \\
\hline & 4 Employees & 0.000 & 0.000 & 0.000 & 0.000 & 0.000 & 0.000 & 0.000 & 0.000 & 0.000 & 0.000 \\
\hline & 5 Parking & 0.000 & 0.000 & 0.000 & 0.000 & 0.000 & 0.000 & 0.000 & 0.000 & 0.000 & 0.000 \\
\hline
\end{tabular}




\begin{tabular}{|c|c|c|c|c|c|c|c|c|c|c|c|c|c|}
\hline & \multicolumn{4}{|c|}{$\begin{array}{l}\text { 4Custom } \\
\text { Groups }\end{array}$} & \multicolumn{3}{|c|}{$\begin{array}{l}\text { Merch- } \\
\text { andise }\end{array}$} & \multicolumn{5}{|c|}{$\begin{array}{l}\text { 6Charact } \\
\text { of Store }\end{array}$} \\
\hline & & $\begin{array}{c}1 \text { White } \\
\text { Collar }\end{array}$ & $\begin{array}{l}2 \text { Blue } \\
\text { Collar }\end{array}$ & 3 Familie & 4 Teens & 1 Low Cost & 2 Quality & 3 Variety & 1Light'ng & 2 Organ. & 3 Clean & $\begin{array}{c}4 \text { Emp- } \\
\text { loyees }\end{array}$ & $5 \mathrm{Pkg}$ \\
\hline \multirow[t]{3}{*}{ 1 Alternat. } & 1 Walmart & 0.036 & 0.038 & 0.036 & 0.040 & 0.033 & 0.030 & 0.032 & 0.036 & 0.024 & 0.031 & 0.035 & 0.086 \\
\hline & 2 KMart & 0.006 & 0.012 & 0.012 & 0.009 & 0.010 & 0.006 & 0.006 & 0.006 & 0.004 & 0.005 & 0.005 & 0.019 \\
\hline & 3 Target & 0.015 & 0.007 & 0.009 & 0.009 & 0.006 & 0.013 & 0.011 & 0.012 & 0.009 & 0.018 & 0.015 & 0.049 \\
\hline \multirow[t]{4}{*}{2 Advertising } & $1 \mathrm{TV}$ & 0.076 & 0.119 & 0.119 & 0.148 & 0.000 & 0.000 & 0.000 & 0.000 & 0.000 & 0.000 & 0.000 & 0.000 \\
\hline & 2 Print Med. & 0.050 & 0.052 & 0.063 & 0.040 & 0.000 & 0.000 & 0.000 & 0.000 & 0.000 & 0.000 & 0.000 & 0.000 \\
\hline & 3 Radio & 0.014 & 0.015 & 0.012 & 0.023 & 0.000 & 0.000 & 0.000 & 0.000 & 0.000 & 0.000 & 0.000 & 0.000 \\
\hline & $\begin{array}{l}\text { 4 Direct } \\
\text { Mail }\end{array}$ & 0.095 & 0.048 & 0.040 & 0.023 & 0.000 & 0.000 & 0.000 & 0.000 & 0.000 & 0.000 & 0.000 & 0.000 \\
\hline \multirow[t]{3}{*}{3 Locations } & 1 Urban & 0.028 & 0.016 & 0.016 & 0.018 & 0.027 & 0.011 & 0.010 & 0.016 & 0.010 & 0.015 & 0.018 & 0.031 \\
\hline & 2 Suburban & 0.141 & 0.047 & 0.052 & 0.052 & 0.012 & 0.062 & 0.064 & 0.071 & 0.051 & 0.074 & 0.073 & 0.135 \\
\hline & 3 Rural & 0.000 & 0.106 & 0.101 & 0.098 & 0.063 & 0.030 & 0.029 & 0.076 & 0.051 & 0.074 & 0.073 & 0.295 \\
\hline \multirow[t]{4}{*}{4 Cust Grps } & 1 White Col. & 0.000 & 0.000 & 0.151 & 0.046 & 0.013 & 0.056 & 0.042 & 0.247 & 0.082 & 0.156 & 0.107 & 0.000 \\
\hline & $\begin{array}{l}2 \text { Blue } \\
\text { Collar }\end{array}$ & 0.000 & 0.000 & 0.350 & 0.096 & 0.028 & 0.040 & 0.042 & 0.247 & 0.082 & 0.134 & 0.107 & 0.000 \\
\hline & 3 Families & 0.463 & 0.463 & 0.000 & 0.590 & 0.156 & $0.14 J$ & 0.157 & 0.119 & 0.257 & 0.318 & 0.400 & 0.000 \\
\hline & 4 Teenagers & 0.077 & 0.077 & 0.039 & 0.000 & 0.055 & 0.013 & 0.012 & 0.031 & 0.019 & 0.036 & 0.031 & 0.000 \\
\hline \multirow[t]{3}{*}{5 Merchand } & 1 Low Cost & 0.000 & 0.000 & 0.000 & 0.000 & 0.000 & 0.477 & 0.477 & 0.000 & 0.000 & 0.000 & 0.000 & 0.000 \\
\hline & 2 Quality & 0.000 & 0.000 & 0.000 & 0.000 & 0.447 & 0.000 & 0.119 & 0.000 & 0.000 & 0.000 & 0.000 & 0.000 \\
\hline & 3 Variety & 0.000 & 0.000 & 0.000 & 0.000 & 0.149 & 0.119 & 0.000 & 0.000 & 0.316 & 0.000 & 0.000 & 0.000 \\
\hline \multirow[t]{5}{*}{ 6Charact. } & 1 Lighting & 0.000 & 0.000 & 0.000 & 0.000 & 0.000 & 0.000 & 0.000 & 0.000 & 0.016 & 0.017 & 0.000 & 0.097 \\
\hline & 2 Organiz. & 0.000 & 0.000 & 0.000 & 0.000 & 0.000 & 0.000 & 0.000 & 0.035 & 0.000 & 0.079 & 0.027 & 0.290 \\
\hline & 3 Cleanli. & 0.000 & 0.000 & 0.000 & 0.000 & 0.000 & 0.000 & 0.000 & 0.092 & 0.044 & 0.000 & 0.110 & 0.000 \\
\hline & 4 Employee & 0.000 & 0.000 & 0.000 & 0.000 & 0.000 & 0.000 & 0.000 & 0.000 & 0.029 & 0.042 & 0.000 & 0.000 \\
\hline & 5 Parking & 0.000 & 0.000 & 0.000 & 0.000 & 0.000 & 0.000 & 0.000 & 0.010 & 0.005 & 0.000 & 0.000 & 0.000 \\
\hline
\end{tabular}

The limit supermatrix is not shown here to save space. It is obtained from the weighted supermatrix by raising it to powers until it converges so that all columns are identical. From the top part of the first column of the limit supermatrix we get the priorities we seek and normalize. These results are compared with the actual values shown in Table 24.

\section{SYNTHESIZED RESULTS FROM THE LIMIT SUPERMATRIX}

The relative market shares of the alternatives Walmart, Kmart and Target from the limit supermatrix are: $0.057,0.024$ and 0.015 . When normalized they are $0.599,0.248$ and 0.154 .

Table 24 The Synthesized Results for the Alternatives

\begin{tabular}{|l|c|c|c|c|}
\hline Alternatives & $\begin{array}{c}\text { Values from } \\
\text { Limit } \\
\text { Supermatrix }\end{array}$ & $\begin{array}{c}\text { Actual } \\
\text { Values } \\
\text { July13, 1998 }\end{array}$ & $\begin{array}{c}\text { Normalized } \\
\text { Values from } \\
\text { Supermatrix }\end{array}$ & $\begin{array}{c}\text { Actual Market Share } \\
\text { as Dollar Sales } \\
\text { Normalized }\end{array}$ \\
\hline Walmart & 0.057 & 58 billion \$ & 0.599 & 54.8 \\
\hline KMart & 0.024 & 27.5 billion \$ & 0.248 & 25.9 \\
\hline Target & 0.015 & 20.3 billion \$ & 0.254 & 19.2 \\
\hline
\end{tabular}

The relative market share values obtained from the model were compared with the actual sales values by computing the compatibility index. The compatibility Index enables is used to determine how close two sets of numbers from a ratio scale or an absolute scale are to each other. 
In this example the result is equal to 1.016 and falls below 1.1 and therefore is an acceptable outcome.

\section{Actual Relative Market Share Based on Sales}

The object was to estimate the market share of Walmart, Kmart, and Target. The normalized results from the model were compared with sales shown in Table 11 as reported in the Discount Store News of July 13, 1998, p.77, of $\$ 58, \$ 27.5$ and $\$ 20.3$ billions of dollars respectively. Normalizing the dollar amounts shows their actual relative market shares to be 54.8, 25.9 and 19.2. The relative market share from the model was compared with the sales values by constructing a pairwise matrix from the results vector in column 1 below and a pairwise matrix from results vector in column 3 and computing the compatibility index using the Hadamard multiplication method. The index is equal to 1.016. As that is about 1.01 the ANP results may be said to be close to the actual relative market share.

\section{Example 2: Estimating Relative Market Share of Airlines (close outcome)}

An ANP model to estimate the relative market share of eight American Airlines is shown in Figure 14. The results from the model and the comparison with the relative actual market share are shown in Table 25.

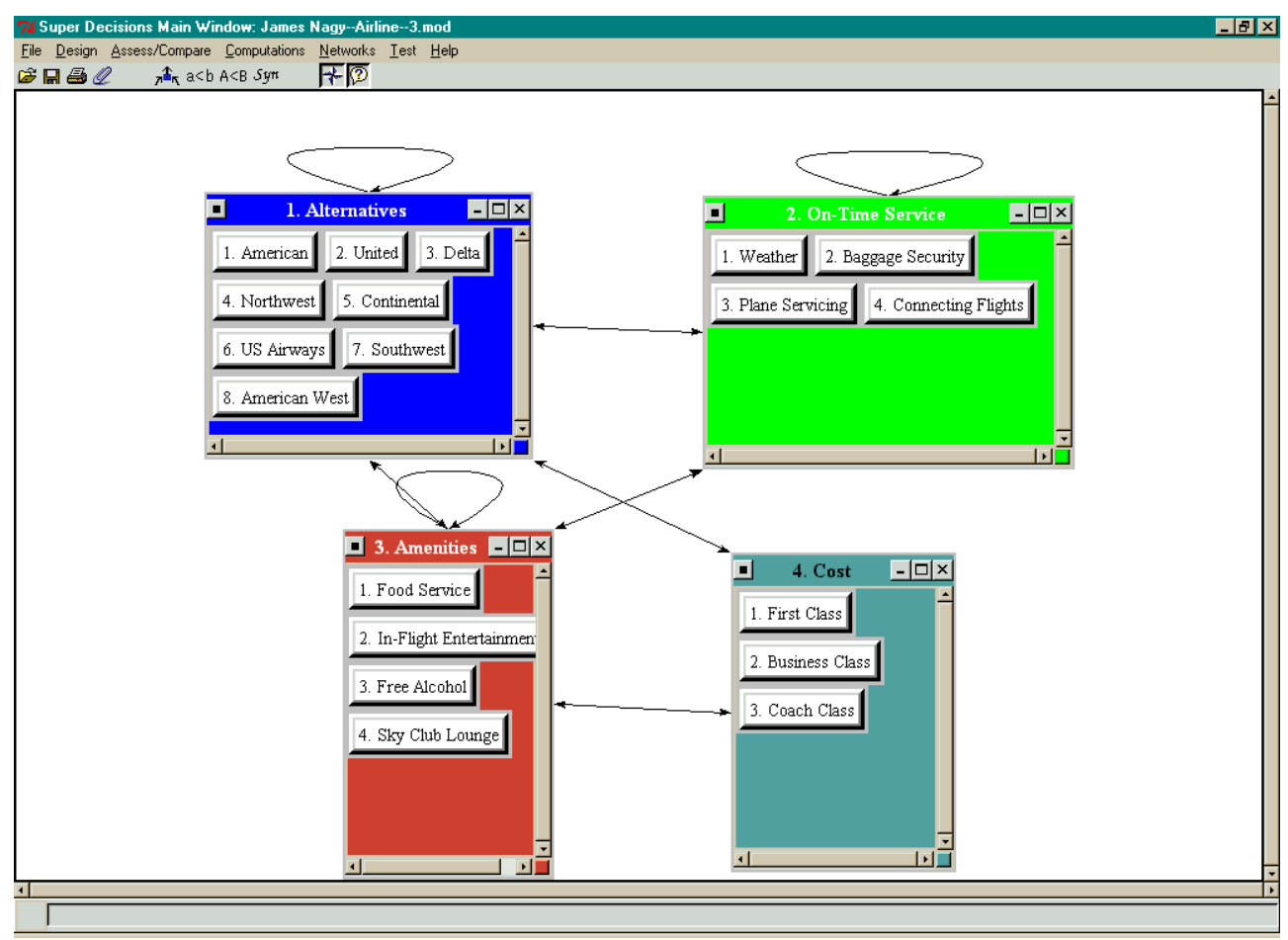

Figure 14 ANP Network to Estimate Relative Market Share of Eight US Airlines 
Table 25 Comparing Model Results with Actual Market Share Data

$\begin{array}{ll}\text { Model } & \text { Actual Market } \\ \text { Results } & \text { Share }\end{array}$

$\begin{array}{lll}\text { American } & 23.9 & 24.0 \\ \text { United } & 18.7 & 19.7 \\ \text { Delta } & 18.0 & 18.0 \\ \text { Northwest } & 11.4 & 12.4 \\ \text { Continental } & 9.3 & 10.0 \\ \text { US Airways } & 7.5 & 7.1 \\ \text { Southwest } & 5.9 & 6.4 \\ \text { American West } & 4.4 & 2.9\end{array}$

We summarize by giving the reader a list of the steps we have followed in applying the ANP.

\section{OUTLINE OF STEPS OF THE ANP}

1. Describe the decision problem in detail including its objectives, criteria and subcriteria, actors and their objectives and the possible outcomes of that decision. Give details of influences that determine how that decision may come out.

2. Determine the control criteria and subcriteria in the four control hierarchies one each for the benefits, opportunities, costs and risks of that decision and obtain their priorities from paired comparisons matrices. If a control criterion or subcriterion has a global priority of $3 \%$ or less, you may consider carefully eliminating it from further consideration. The software automatically deals only with those criteria or subcriteria that have subnets under them. For benefits and opportunities, ask what gives the most benefits or presents the greatest opportunity to influence fulfillment of that control criterion. For costs and risks, ask what incurs the most cost or faces the greatest risk. Sometimes (very rarely), the comparisons are made simply in terms of benefits, opportunities, costs, and risks in the aggregate without using control criteria and subcriteria.

3. Determine the most general network of clusters (or components) and their elements that apply to all the control criteria. To better organize the development of the model as well as you can, number and arrange the clusters and their elements in a convenient way (perhaps in a column). Use the identical label to represent the same cluster and the same elements for all the control criteria.

4. For each control criterion or subcriterion, determine the clusters of the general feedback system with their elements and connect them according to their outer and inner dependence influences. An arrow is drawn from a cluster to any cluster whose elements influence it.

5. Determine the approach you want to follow in the analysis of each cluster or element, influencing (the preferred approach) other clusters and elements with respect to a criterion, or being influenced by other clusters and elements. The sense (being influenced or influencing) must apply to all the criteria for the four control hierarchies for the entire decision.

6. For each control criterion, construct the supermatrix by laying out the clusters in the order they are numbered and all the elements in each cluster both vertically on the left and horizontally at the 
top. Enter in the appropriate position the priorities derived from the paired comparisons as subcolumns of the corresponding column of the supermatrix.

7. Perform paired comparisons on the elements within the clusters themselves according to their influence on each element in another cluster they are connected to (outer dependence) or on elements in their own cluster (inner dependence). In making comparisons, you must always have a criterion in mind. Comparisons of elements according to which element influences a given element more and how strongly more than another element it is compared with are made with a control criterion or subcriterion of the control hierarchy in mind.

8. Perform paired comparisons on the clusters as they influence each cluster to which they are connected with respect to the given control criterion. The derived weights are used to weight the elements of the corresponding column blocks of the supermatrix. Assign a zero when there is no influence. Thus obtain the weighted column stochastic supermatrix.

9. Compute the limit priorities of the stochastic supermatrix according to whether it is irreducible (primitive or imprimitive [cyclic]) or it is reducible with one being a simple or a multiple root and whether the system is cyclic or not. Two kinds of outcomes are possible. In the first all the columns of the matrix are identical and each gives the relative priorities of the elements from which the priorities of the elements in each cluster are normalized to one. In the second the limit cycles in blocks and the different limits are summed and averaged and again normalized to one for each cluster. Although the priority vectors are entered in the supermatrix in normalized form, the limit priorities are put in idealized form because the control criteria do not depend on the alternatives.

10. Synthesize the limiting priorities by weighting each idealized limit vector by the weight of its control criterion and adding the resulting vectors for each of the four merits: Benefits (B), Opportunities $(\mathrm{O})$, Costs $(\mathrm{C})$ and Risks $(\mathrm{R})$. There are now four vectors, one for each of the four merits. An answer involving marginal values of the merits is obtained by forming the ratio $\mathrm{BO} / \mathrm{CR}$ for each alternative from the four vectors. The alternative with the largest ratio is chosen for some decisions. Companies and individuals with limited resources often prefer this type of synthesis.

11. Governments prefer this type of outcome. Determine strategic criteria and their priorities to rate the four merits one at a time. Normalize the four ratings thus obtained and use them to calculate the overall synthesis of the four vectors. For each alternative, subtract the costs and risks from the sum of the benefits and opportunities. At other times one may subtract the costs from one and risks from one and then weight and add them to the weighted benefits and opportunities. This is useful for predicting numerical outcomes like how many people voted for an alternative and how many voted against it. In all, we have three different formulas for synthesis.

12. Perform sensitivity analysis on the final outcome and interpret the results of sensitivity observing how large or small these ratios are. Can another outcome that is close also serve as a best outcome? Why? By noting how stable this outcome is. Compare it with the other outcomes by taking ratios. Can another outcome that is close also serve as a best outcome? Why?

The next section includes real ANP applications of many different areas from business to public policy. We intentionally included not only simple examples that have a single network such as market share examples but also more complicated decision problems. The second group includes BOCR merit evaluations using strategic criteria, with control criteria (and perhaps subcriteria) under them for each of the BOCR and their related decision networks. 
In the light of the above explanations, we cover a rather wide spectrum in the variety of examples. There are examples that do not have BOCR merit evaluation and/or sensitivity analysis while some of them analyze the problem with all the details. In order to start with a clear understanding, we preferred to give the example below that has all the possible analyses from the BOCR merit evaluation to the control criteria and subcriteria, decision networks for each of these and sensitivity analysis with regard to the BOCR.

\section{A COMPLETE BOCR EXAMPLE}

Disney Decision: A New Theme Park in Greater China (By Amber Ling-Hui, Lin SzuLun Peng)

\section{Introduction / Background}

In order to enhance operations in foreign market, Disney is constantly searching for areas where it can expand into new markets. According to the projected number of foreign visitors, Walt Disney World expects to increase the current level from 20 percent foreign visitors in domestic parks to 50 percent as well as to expand its theme park business outside the U.S. To achieve these projected numbers Disney needs to make an aggressive attempt to expand its presence in foreign markets, especially Greater China. However, considering the diverse social and economic backgrounds within this area, Disney needs to carefully evaluate the possible benefits as well as the costs and potential risks. In this model, we narrow down the alternatives to Hong Kong, Shanghai, Taiwan and no investment in Greater China. In fact, an awakening and growing middle class in these three areas is exactly the prime target audience for a Disney theme park.

\section{Ultimate goal for Disney}

Disney's intention is to make a minimal equity investment in any operating entity and generate most of its returns through royalty, licensing, and fee income streams.

\section{Main Model}

\section{BOCR Networks and Cluster Definitions}

Under the benefits, opportunities, costs, and risks (BOCR) models, different clusters define interactions with respect to the control hierarchy established. The benefits networks indicate the alternatives that yield the most benefit and the opportunities networks indicate the alternative that offers the most opportunities, whereas the costs and risks networks indicate the alternatives that are the most costly or pose the most risk on each alternative.

The flow of the decision process is to first build the networks and sub-networks for each of the BOCR models (Figure 15), make the judgments and evaluate which is the best alternative in each case for this particular decision. The importance of the BOCR must then be determined by rating them with respect to the strategic criteria of the organization or decision maker. 


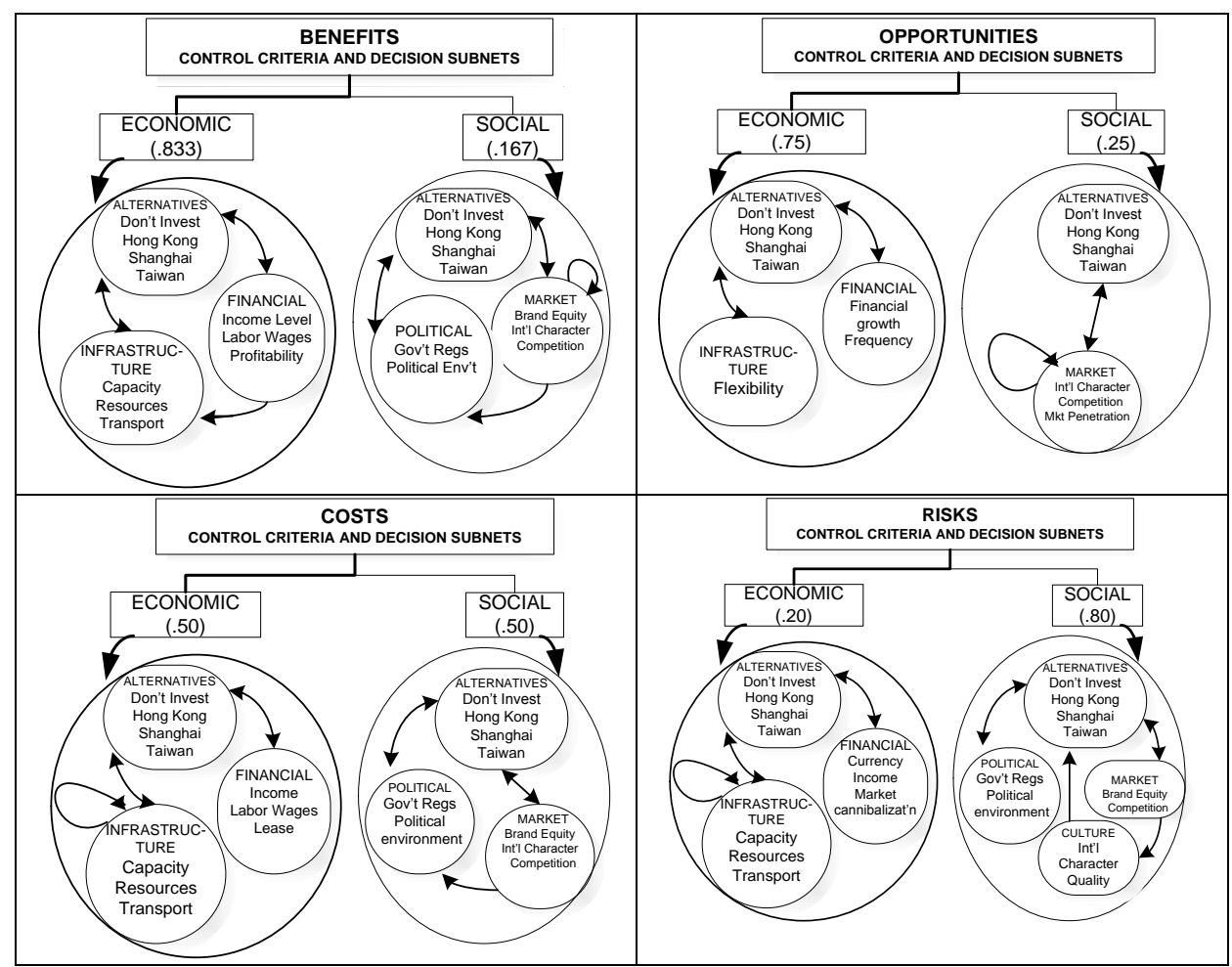

Figure 15 Decision Sub-networks with Clusters and Nodes for each of the BOCR

\section{Control Criteria and Subnets of the BOCR}

Each of the BOCR has control criteria whose priorities are established through pairwise comparison. The control criteria in turn have associated network sub-models that contain the alternatives of the decision and clusters of elements. Thus priorities for the alternatives are determined in each of the subnets. These are weighted by their control criterion, and these results are multiplied by the BOCR weights from the rating model and combined to give the final results. The alternatives appear in a cluster in every decision subnet, so we define them only once here. There are three locations being considered for the first Disney theme park in Greater China plus the alternative of not building at all.

\section{Alternatives (in every subnet)}

\section{- Don't invest in Greater China}

\section{- Hong Kong}

\section{- Shanghai}

\section{- Taiwan}

Moving on to the first subnet, under the Social control criterion for Benefits we show the clusters in that network below:

\section{Clusters in Benefits/Social Subnet}

\section{- Alternatives}




\section{- Market}

Brand Equity: For the brand equity, we consider it as an intangible asset to Walt Disney. Brand equity represents Disney's reputation and image in the market. Within this subnet, we will examine how much benefit each alternative can bring to Disney in terms of increasing their brand equity.

International Character: International character refers to having a diversified visitor base. The higher the diversification of the visitor base, the more it benefits Disney.

Market Competition: Market competition refers to the number of competitors with comparable scale in one market. Within the benefit cluster, we will discuss the level that Disney can benefit from the competition in the market under each alternative.

\section{- Political Factors}

Government Regulation: We believe a favorable local government regulation on the theme park business will definitely benefit Disney's operation in that area and vise versa.

Political Environment: We believe a stable political environment will create a promising investment environment. Thus, the benefits will be measured base on the current political stability and potential political instability of each alternative.

\section{Interactions between Clusters in the Benefits/Social subnet}

In this subnet, we can see the interactions among clusters as well as interactions within clusters.

Market Factors: First of all, since the government regulations and political environment will affect the international character and the market competition in a market, we can see an interaction between market cluster and political factors cluster. Besides, different choices that Disney makes will affect the company itself in terms of brand equity, international character and competition in the market. Finally, the competitive ability of the company and the international character of the market may also affect Disney's brand equity at the end. Thus, we can see another interaction within the market cluster itself.

Political Factors: Besides the interaction with the market cluster, the political factors cluster also interacts with the alternative cluster because the political factors are also affected by different alternatives.

Alternatives: While each alternative affects factors in the market and political clusters, those factors also have effect on Disney's decision among alternatives in return. Thus, there are also backward interactions between the alternatives cluster and the other two clusters.

\section{Nodes in the Benefits/Economic Subnet Clusters}

- Alternatives

- Financial Factors 
Gross and disposable income level: Under this factor, only the current gross and disposable income level of the area's citizens will be considered. We assume that a higher income level in the local area will bring more business to the Disney facility and further increase Disney's revenue.

Labor Wage: Labor refers to the current level of local labor wage. A lower labor wage will benefit Disney from reducing operating overheads.

Profitability: Profitability refers to the forecasted profits based on the current market situation.

\section{- Infrastructure}

Accommodation Capacity: This refers to the current hotel accommodation capacity of that area.

Resources: The resources factor refers to the current construction quality and efficiency of the area.

Transportation: Transportation here means the current development of local railroads, airports, tunnels, etc. If the area is already well developed, Disney can benefit from an instant resource of transportation system for customers.

\begin{tabular}{|c|c|c|c|c|c|}
\hline Graphic & Alternatives & $\begin{array}{l}\text { Total from } \\
\text { Supermatrix }\end{array}$ & $\begin{array}{l}\text { Total } \\
\text { Normalized }\end{array}$ & $\begin{array}{l}\text { Total } \\
\text { Idealized }\end{array}$ & Ranking \\
\hline 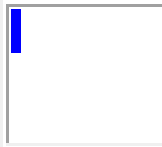 & $\begin{array}{l}\text { Don't invest } \\
\text { in } \\
\text { China }\end{array}$ & 0.0273 & 0.0579 & 0.1242 & 4 \\
\hline & Hong Kong & 0.2201 & 0.4662 & 1.0000 & 1 \\
\hline & Shanghai & 0.1379 & 0.2922 & 0.6267 & 2 \\
\hline & Taiwan & 0.0867 & 0.1837 & 0.3940 & 3 \\
\hline
\end{tabular}

Table 27 Alternative Rankings from the Benefits/Social Subnet

\begin{tabular}{|l|l|l|l|l|l|l|}
\hline Graphic & Alternatives & Total & Normalized & Idealized & Ranking \\
\hline & $\begin{array}{l}\text { Don't invest in } \\
\text { Greater China }\end{array}$ & 0.0045 & 0.0099 & 0.0219 & 4 \\
\hline & Hong Kong & 0.2059 & 0.4521 & 1.0000 & 1 \\
\hline \hline & Shanghai & 0.1556 & 0.3417 & 0.7558 & 2 \\
\hline \hline & Taiwan & 0.0894 & 0.1963 & 0.4342 & 3 \\
\hline
\end{tabular}

Combining the outcomes from the social and economic decision subnets for the benefits model produces the results shown below. The normalized values (in bold) show that Hong Kong offers the most benefits, and by a significant amount, at $46.4 \%$. 
Table 28 Synthesized Result for the Benefits Model

\begin{tabular}{|l|l|l|l|l|l|l|}
\hline Graphic & Alternatives & Total & Normal & Ideal & Ranking \\
\hline \hline & Don't invest in Greater China & 0.107 & $\mathbf{0 . 0 5 0}$ & 0.107 & 4 \\
\hline \hline & Hong Kong & 1.0000 & $\mathbf{0 . 4 6 4}$ & 1.000 & 1 \\
\hline & Shanghai & 0.648 & $\mathbf{0 . 3 0 1}$ & 0.648 & 2 \\
\hline \hline & Taiwan & 0.401 & $\mathbf{0 . 1 8 6}$ & 0.401 & 3 \\
\hline
\end{tabular}

In the opportunities, costs and risks models, the decision subnets are built based on the same logic as that of the benefits subnets. The details of their clusters and nodes are similar to that of benefits and will not be shown here. A general idea of what they are can be obtained from the figure above showing the decision sub-networks. The results for each of the control criteria for opportunities, costs and risks are given below.

We show only the final synthesized results for opportunities, costs, and risks

Table 29 Synthesized Results for the Opportunities Model
\begin{tabular}{|l|l|l|l|l|l|l|l|}
\hline Graphic & Alternatives & Total & Normal & Ideal & Ranking \\
\hline & Don't invest in Greater China & 0.019 & $\mathbf{0 . 0 1 0}$ & 0.019 & 4 \\
\hline & Hong Kong & 0.428 & $\mathbf{0 . 2 2 4}$ & 0.428 & 3 \\
\hline & Shanghai & 1.000 & $\mathbf{0 . 5 2 4}$ & 1.000 & 1 \\
\hline \hline & Taiwan & 0.462 & $\mathbf{0 . 2 4 2}$ & 0.462 & 2 \\
\hline \hline
\end{tabular}

\begin{tabular}{|l|l|l|l|l|l|l|}
\hline Table 30 Synthesized Results for the Costs Model & \multicolumn{3}{l|}{} \\
\hline Graphic & Alternatives & Total & Normal & Ideal & Ranking \\
\hline & Don't invest in Greater China & 0.104 & $\mathbf{0 . 0 4 0}$ & 0.105 & 4 \\
\hline & Hong Kong & 0.610 & $\mathbf{0 . 2 3 3}$ & 0.617 & 3 \\
\hline & Shanghai & 0.989 & $\mathbf{0 . 3 7 8}$ & 1.000 & 1 \\
\hline & Taiwan & 0.912 & $\mathbf{0 . 3 4 9}$ & 0.922 & 2 \\
\hline
\end{tabular}

Table 31 Synthesized Results for the Risks Model

\begin{tabular}{|l|l|l|l|l|l|}
\hline Graphic & Alternatives & Total & Normal & Ideal & $\begin{array}{l}\text { Rank } \\
\text { ing }\end{array}$ \\
\hline & $\begin{array}{l}\text { Don't invest in Greater } \\
\text { China }\end{array}$ & 0.116 & $\mathbf{0 . 0 5 1}$ & 0.118 & 4 \\
\hline & Hong Kong & 0.425 & $\mathbf{0 . 1 8 8}$ & 0.434 & 3 \\
\hline \hline & Shanghai & 0.981 & $\mathbf{0 . 4 3 4}$ & 1.000 & 1 \\
\hline \hline & Taiwan & 0.736 & $\mathbf{0 . 3 2 6}$ & 0.751 & 2 \\
\hline
\end{tabular}




\section{Decision Model for Rating Strategic Criteria}

The final step in the decision is to determine the strategic criteria that are more or less the same for the organization or individual in making any decision and use them to rate the BOCR with respect to competition, income level, infrastructure, international character and political support as shown in the table below. We thought the five strategic criteria below pretty well captured Disney's main corporate concerns about their theme parks.

To prepare to rate the strategic criteria one first pairwise compares them for importance in a hierarchy resulting in the priorities shown underneath their names in Table 20. Then one establishes intensities to indicate the degree of fulfillment (in the case of benefits and opportunities) or impact (in the case of costs and risks). The intensities and their priorities (in the ideal form) are Very Strong(1.000), Strong(.627), Medium(.382), Moderate(.232) and Weak(.148). Priorities are determined for them by pairwise comparing. In this case the same intensities and priorities are used for each strategic criterion, although they could be different.

\section{Strategic Criteria Definitions}

The strategic criteria are defined below and pairwise compared for importance with respect to Disney's goal. Ratings are then established for each of these criteria and pairwise compared to establish their priorities in turn. These ratings are then used to determine the priority or importance of Benefits, Opportunities, Costs and Risks and these values are used to weight the results in the submodels attached to them.

Competition - Successful theme parks in the area of the Disney Facility may be viewed both positively and negatively. Other theme parks already in the areas represent competition for Disney; however, competitors may also bring more people to the area to visit both facilities at the same time.

Income Level- Gross and disposable income levels of the area's citizens may also affect the success of the park. Consider Tokyo Disney Land for example. Approximately $95 \%$ of its visitors are local Japanese; thus, the high average income level of Japanese does appear to contribute to the tremendous success of Disney in Japan.

Infrastructure- Infrastructure in the area of the park and the regional support are also important. Visitors should be able to access the park easily. The transportation system should be well established or enhanced while the park is being constructed. A good area should have the infrastructure to support a park efficiently. Besides, the region should also contribute to extending the time visitors are able to spend at the Disney facilities. For example, a stock of hotel rooms to support park visitors is important and rooms at a variety of price levels, from economy all the way to luxury, should be available when the park opens.

International Character - Disney is looking for "international character" for any theme park it builds in Greater China. A diversified visitor base will reduce the risks of problems in one country having an adverse effect on the flow of international visitors. 
Political Support - In all Disney's international operations, support from local government is critical to the Disney Company. This support ranges from providing a good location to build the theme park to insuring sufficient capital flow.

\section{Rating the Benefits, Opportunities, Costs and Risks}

To select the ratings in Table 32 for the Benefits, for example, one must keep in mind the top alternative in the synthesized results for the benefits model given in Table 28 that has the highest priority, Hong Kong. For example, Hong Kong's benefits to fulfill the Competition strategic criterion or objective is thought to be strong. For fulfilling benefits for Income Level, Hong Kong would be very strong as people there have high disposable income, and so on for all the Strategic Criteria.

When making ratings for Costs and Risks, keep in mind that the highest priority alternative is the most costly or most risky. To select the ratings for Risks keep in mind Shanghai. Shanghai has very strong risks so far as Competition is concerned, and strong risks for Income Level as people have less disposable income there, and medium risks for Political Support which means the risk is not too great for Disney in Shanghai as they believe they would have the support of the Chinese Government.

The overall priorities for each of the BOCR are computed by multiplying and adding across each row and normalizing the final result shown in the last column of Table 32. The priorities show that the most important merit is Benefits at $31.9 \%$ followed by Opportunities at $26.4 \%$. This means that the priorities of the alternatives under benefits are weighted more heavily. Benefits at $31.9 \%$ drive the decision more than the Risks at $19.3 \%$.

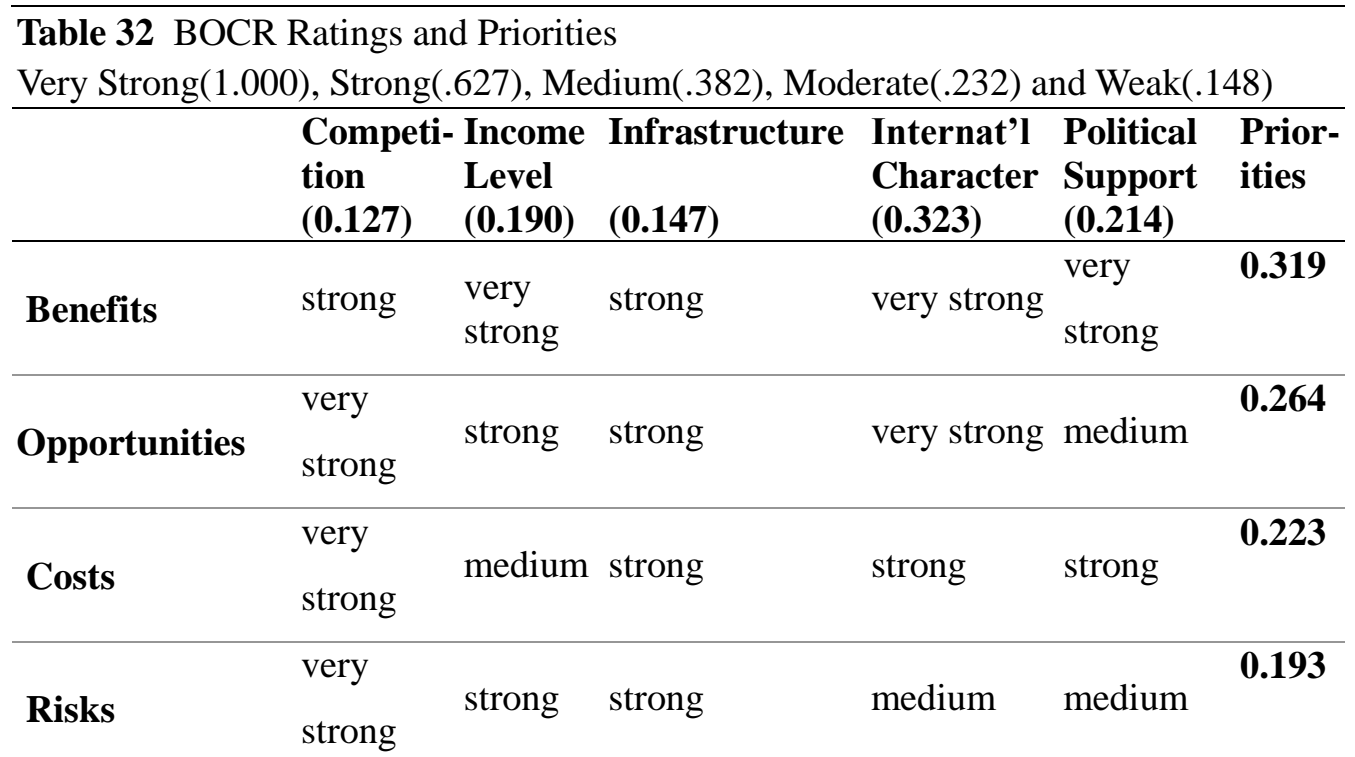

The final results shown in Table 33 are obtained using the formula $b B+o O-c C-r R$ where $b, o$, $\mathrm{c}$ and $\mathrm{r}$ are the priorities for Benefits, Opportunities, Costs and Risks just obtained from rating the with respect to the strategic criteria in Table 32. This formula is applied to the alternatives using the priority vectors from the synthesized results (the $\mathrm{B}, \mathrm{O}, \mathrm{C}$, and $\mathrm{R}$ of the formula) in the previous tables. Since this formula involves negatives, the overall synthesized results in Table 33 may be negative, meaning that the alternative is undesirable. Sometimes all results are negative, 
and one is forced to take the least undesirable one. In Table 33 positive results are labeled in blue and negative ones in red. Here Hong Kong is the best with the highest positive value and Taiwan is the worst with the highest negative value.

\begin{tabular}{|c|c|c|c|c|c|}
\hline Graphic & Alternatives & Total & Normal & Ideal & Ranking \\
\hline (red) & $\begin{array}{l}\text { Don't invest } \\
\text { in Greater } \\
\text { China }\end{array}$ & - & -0.017 & 0.030 & 3 \\
\hline (blue) & Hong Kong & 0.214 & 0.567 & 1.000 & 1 \\
\hline (blue) & Shanghai & 0.061 & 0.161 & 0.284 & 2 \\
\hline (red) & Taiwan & - 0.096 & -0.255 & - & 4 \\
\hline
\end{tabular}

As we can see, from the overall synthesized results in Table 33, Disney's best option is to build their new theme park in Hong Kong.

\section{Sensitivity Analysis Graphs}

Sensitivity analysis in Figure 16 shows that when the importance of benefits is greater than 0.05 , investing in Hong Kong is the best choice. The dotted vertical line indicates the priority of Benefits, for example. At a priority of less than about 0.35 for opportunities, Hong Kong is the best choice, but above that the choice shifts to Shanghai. One might interpret this as meaning that there are great opportunities in Shanghai, but it is also risky as can be seen from the risks sensitivity graph. As the priority of costs increases beyond about 0.38 , the best choice shifts from investing in Hong Kong to not investing at all. As the importance of risk increases the preferred alternative is to not to invest as all in Greater China, but since the priority is negative, below the $\mathrm{x}$-axis, this is not a particularly good alternative, though it is the least negative. When risk is less than about 0.50, the preferred alternative is to invest in Hong Kong.

\begin{tabular}{|c|c|}
\hline BENEFITS & OPPORTUNITIES \\
\hline $\begin{array}{l}\text { At Benefits }=32.9 \% \text {, Hong Kong (top } \\
\text { line) is best; Shanghai second and } \\
\text { Taiwan (bottom line) is worst }\end{array}$ & $\begin{array}{l}\text { At Opportunities }=26.4 \% \text {, Hong Kong } \\
\text { (top line) is best; Shanghai is second } \\
\text { and Taiwan (bottom line) is worst }\end{array}$ \\
\hline
\end{tabular}




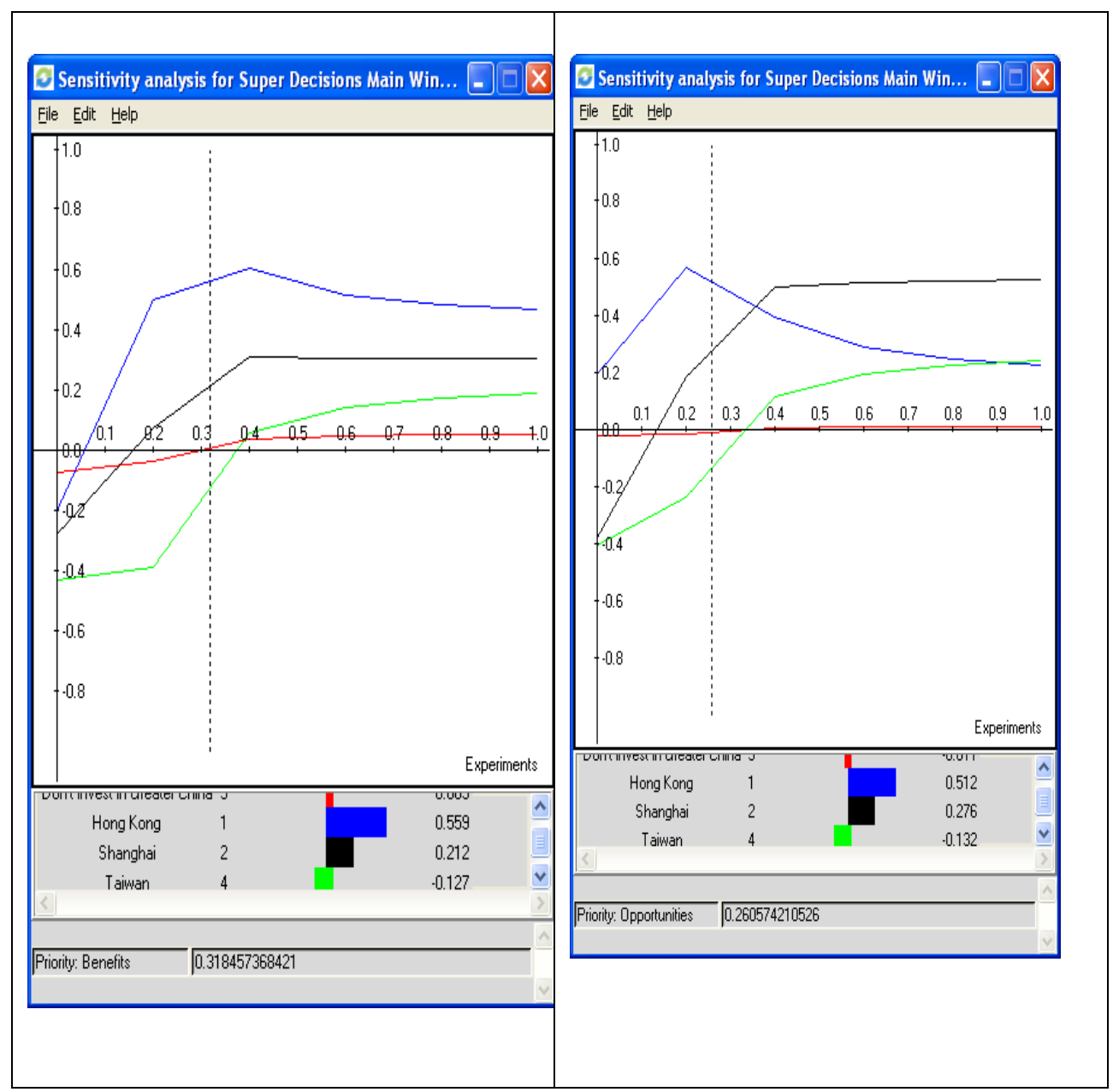

Figure 16 Sensitivity Graphs for Benefits and Opportunities

The vertical dotted line represents the priority of Benefits and Opportunities. To see what happens as the importance of Benefits increases, move the vertical line to the right. Above a Benefits priority of about $40 \%$ the least preferred alternative changes from Taiwan to Don't Invest in Greater China. The line immediately under Hong Kong at down is Shanghai. One might interpret this as indicating that investing in China somewhere is imperative in terms of benefits.

As the importance of Opportunities increases past about 35\%, the top line would be Shanghai and the bottom line Taiwan. This can be interpreted to mean that the greatest opportunities lie in Shanghai. Similar conclusions can be made from the costs and risks graphs in Figure 17. 

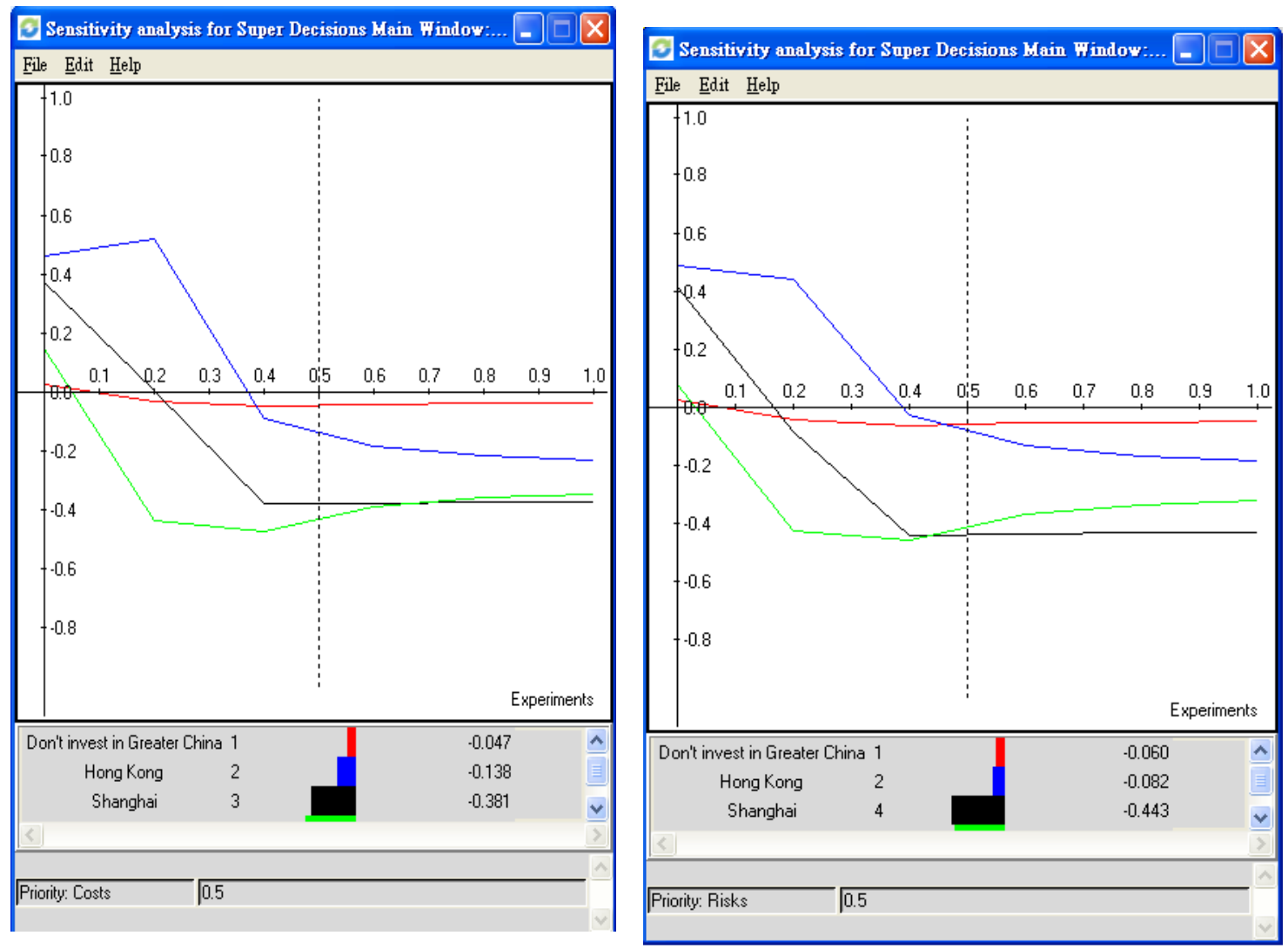

Figure 17 Sensitivity Graphs for Costs and Risks

In Figure 17 (left part) at an importance of $22.3 \%$ for Costs, Hong Kong (the top line) is most costly and Taiwan (the bottom line) is least costly, perhaps because of the political uncertainty and lack of supporting infrastructure in Hong Kong, and as Costs increases, not investing in China is the top line (after about 40\%). So it is extremely risky to not invest in China at all.

In Figure 17 (right part) at an importance of $19.3 \%$ for Risks the top line is Hong Kong, so it is most risky and the bottom line is Taiwan, meaning least risky.

In sum, the greatest benefits and opportunities lie in mainland China, along with the greatest costs and risks, but netting it out, Hong Kong is best overall. 


\section{Decision on National Missile Defense (NMD) - An Application of the ANP with Strong Risks (Analysis done in 2000, decision made to implement in December 2002)}

Not long ago, the United States government faced the crucial decision of whether or not to commit itself to the deployment of a National Missile Defense (NMD) system. Many experts in politics, the military, and academia had expressed different views regarding this decision. The most important rationale behind supporters of the NMD system was protecting the U.S. from potential threats said to come from countries such as North Korea, Iran and Iraq. According to the Central Intelligence Agency, North Korea's Taepo Dong long-range missile tests were successful, and it has been developing a second generation capable of reaching the U.S. Iran also tested its medium-range missile Shahab-3 in July 2000. Opponents expressed doubts about the technical feasibility, high costs (estimated at $\$ 60$ billion), political damage, possible arms race, and the exacerbation of foreign relations.

The idea for the deployment of a ballistic missile defense system has been around since the late 1960s but the current plan for NMD originated with President Reagan's Strategic Defense Initiative (SDI) in the 1980s. SDI investigated technologies for destroying incoming missiles. The controversies surrounding the project were intensified with the National Missile Defense Act of 1996, introduced by Senator Sam Nunn (D-GA) in June 25, 1996. The bill required Congress to make a decision on whether the U.S. should deploy the NMD system by 2000 . The bill also targeted the end of 2003 as the time for the U.S. to be capable of deploying NMD. The idea explored in this project is to develop and illustrate the three phases with a timely example, the intricate and very costly decision regarding a National Missile Defense (NMD) system. Because of the possibility of dependence and feedback, we use the Analytic Network Process (ANP) and its software Super-Decisions with its sensitivity analysis option to examine the NMD decision. On February 21, 2002 this author gave a half-day presentation on the subject to National Defense University in Washington. In December 2002, President George W. Bush and his advisors decided to build the NMD. This study may have had no influence on the decision but still two years earlier (September 2000) it had arrived at the same outcome. The alternatives we considered for this analysis are: Deploy NMD, Global defense, R\&D, Termination of the NMD program.

\section{CRITERIA AND DECISION NETWORKS [3]}

The second column of Table shows the criteria of each BOCR. For example, there are four benefits criteria: Economic (0.157), Political (0.074), Security (0.481) and Technology (0.288). The priorities attached to each are obtained through pairwise comparisons. Each criterion under benefits has subcriteria such as Local Economy and Defense Industry under Economic. Again, the priorities of the two subcriteria are obtained from pairwise comparisons and similarly for the remaining criteria and subcriteria under opportunities, costs and risks. Opportunities and risks have no subcriteria. The total number of criteria and subcriteria used as control criteria for the comparisons made in the networks is 2 . The global priorities of these criteria (subcriteria) shown in the last column of Table are obtained by weighting their priorities by those of their parent criterion if there is one. For example, for local economy we have $.157 \times .141=.022$. We will see later, after the BOCR merits are weighted, that the priorities of nine of these (shown in boldface), Military Capability, Technological Advancement, Arms Sales, Spin-Off, Security Threat, Sunk Cost, Further Investment, Arms Race, and Technical Failure account for approximately 0.760 of the total. To economize effort, we used these nine as control criteria each with its decision network to do the analysis. Actually we simply chose the top ones under each merit without 
being fully consistent about the cutoff point. For example we left out U.S. Reputation under Risks. All economic cost factors were included. We proceeded as if these nine criteria and subcriteria, called covering criteria for the alternatives, were the only criteria to drive the outcome. Their decision networks and connections are shown in Figures 18 to 26 . A more thorough analysis might include a few more criteria or subcriteria.

\begin{tabular}{|c|c|c|c|}
\hline \multicolumn{4}{|c|}{ Table 34 Criteria and Their Priorities } \\
\hline Merits & Criteria & Sub-criteria & $\begin{array}{l}\text { Global } \\
\text { Priorities } \\
\text { (Normalized) }\end{array}$ \\
\hline \multirow[t]{9}{*}{ Benefits } & \multirow{2}{*}{$\begin{array}{l}\text { Economic } \\
(0157)\end{array}$} & Local Economy (0.141) & .022 \\
\hline & & Defense Industry (0.859) & .135 \\
\hline & \multirow{2}{*}{$\begin{array}{l}\text { Political } \\
(0.074)\end{array}$} & Bargaining Power (0.859) & .064 \\
\hline & & U.S. Military Leadership & .010 \\
\hline & \multirow{3}{*}{$\begin{array}{l}\text { Security } \\
(0.481)\end{array}$} & Deterrence $(0.267)$ & .128 \\
\hline & & Military Capability (0.590) & .284 \\
\hline & & Anti-terrorism $(0.143)$ & .069 \\
\hline & \multirow{2}{*}{$\begin{array}{l}\text { Technolog } \\
\mathrm{y}(0.288)\end{array}$} & Tech. Advancement (0.834) & .240 \\
\hline & & Tech. Leadership (0.166) & .048 \\
\hline \multirow[t]{4}{*}{ Opportunities } & & Arms Sales $(0.520)$ & .520 \\
\hline & & Spin- off (0.326) & .326 \\
\hline & & Space Development $(0.051)$ & .051 \\
\hline & & Protection of Allies (0.103) & .103 \\
\hline \multirow[t]{5}{*}{ Costs } & \multicolumn{2}{|c|}{ Security Threat: Vulnerability to the security } & .687 \\
\hline & \multirow{2}{*}{$\begin{array}{l}\text { Economic } \\
(0.228)\end{array}$} & Sunk Cost (0.539) & .12 \\
\hline & & Further Investment $(0.461)$ & .105 \\
\hline & \multirow{2}{*}{$\begin{array}{l}\text { Political } \\
(0.085)\end{array}$} & ABM Treaty (0.589) & .050 \\
\hline & & Foreign Relations $(0.411)$ & .035 \\
\hline \multirow[t]{5}{*}{ Risks) } & & Technical Failure (0.430) & .430 \\
\hline & & Arms Race (0.268) & .268 \\
\hline & & Increased Terrorism $(0.052)$ & .052 \\
\hline & & Environmental & .080 \\
\hline & & U.S. Reputation (0.170) & .170 \\
\hline
\end{tabular}




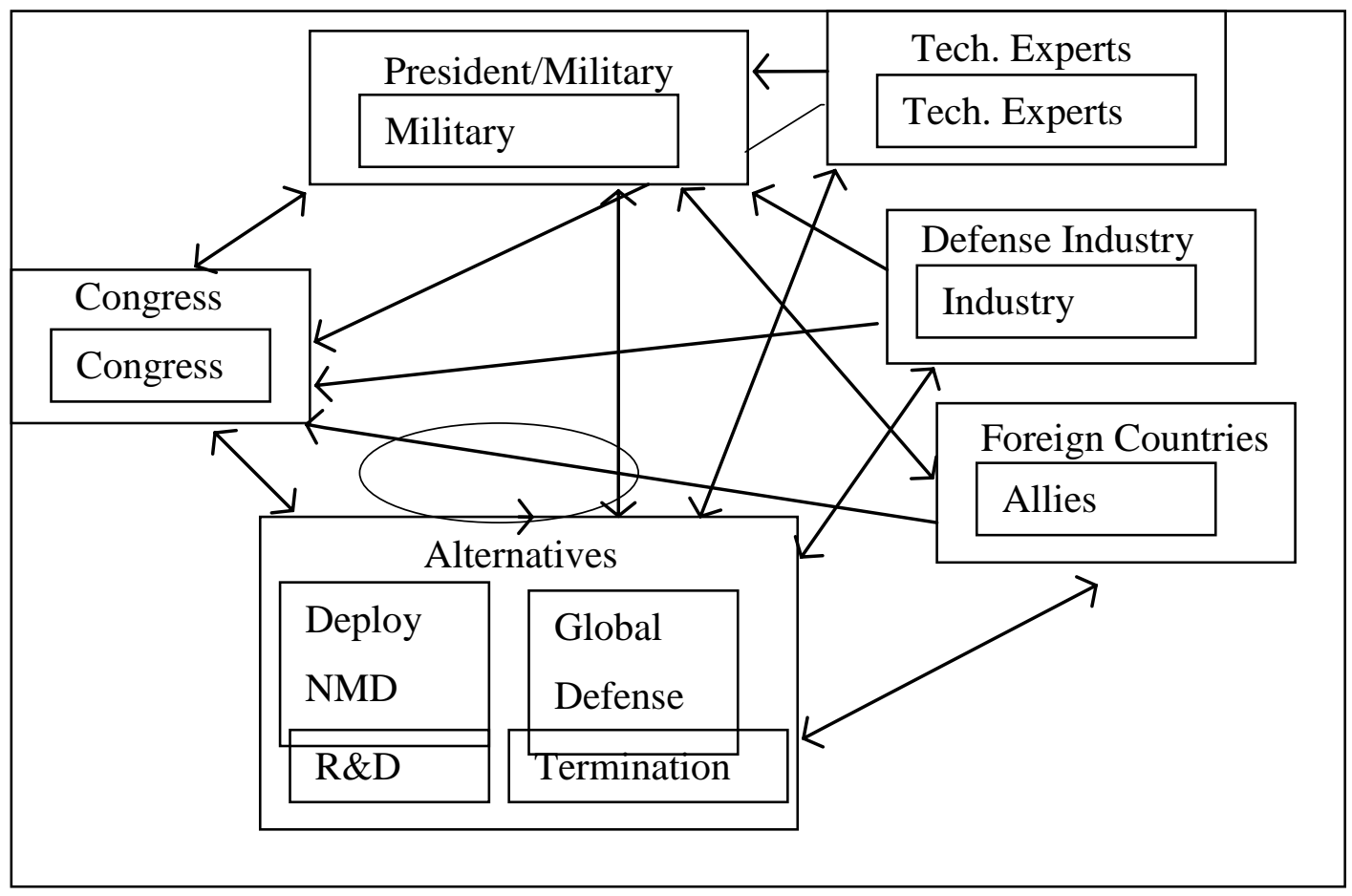

Figure 18 Decision Network under the Military Capability

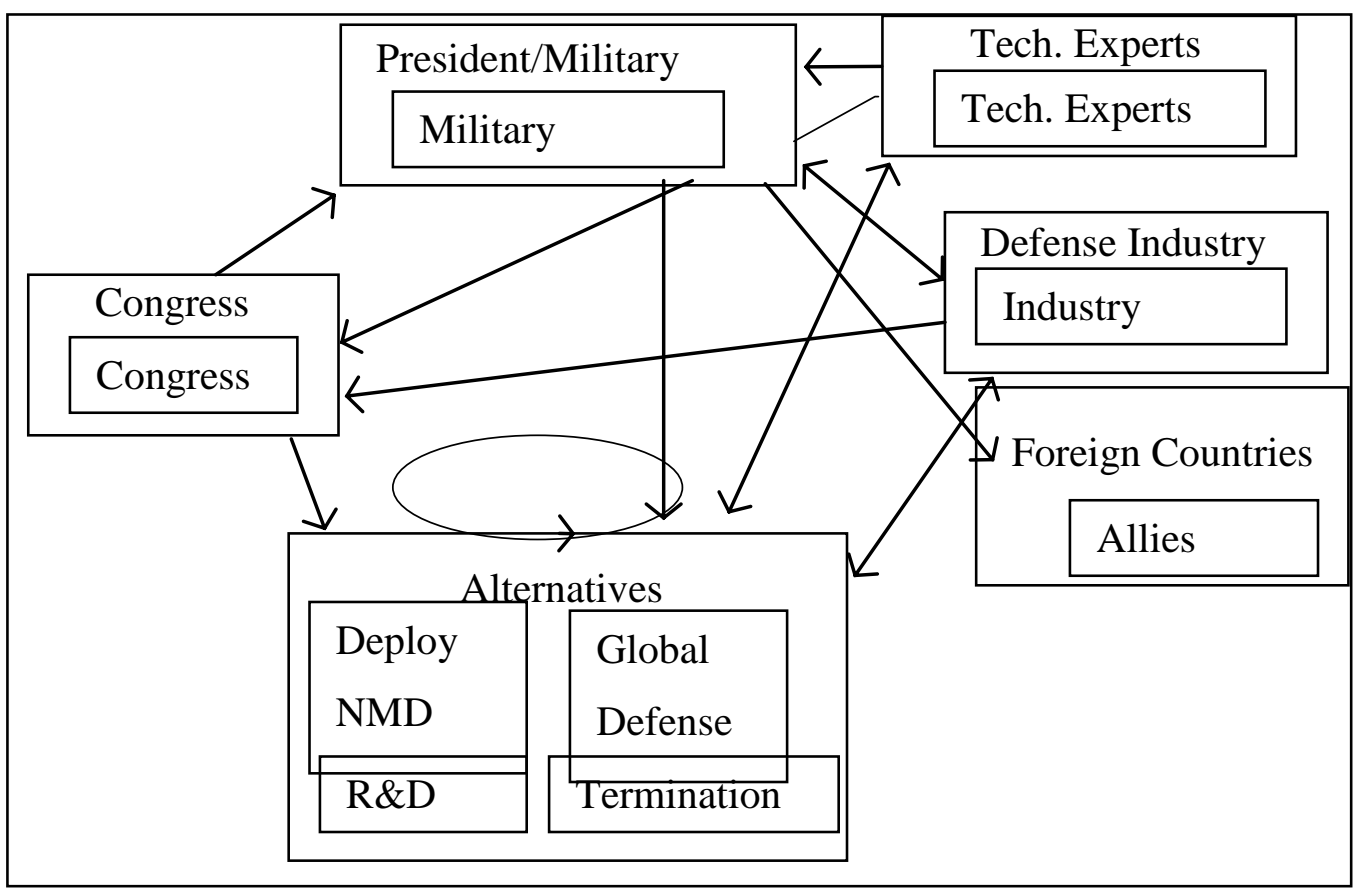

Figure 19 Decision Network under the Technological Advancement 


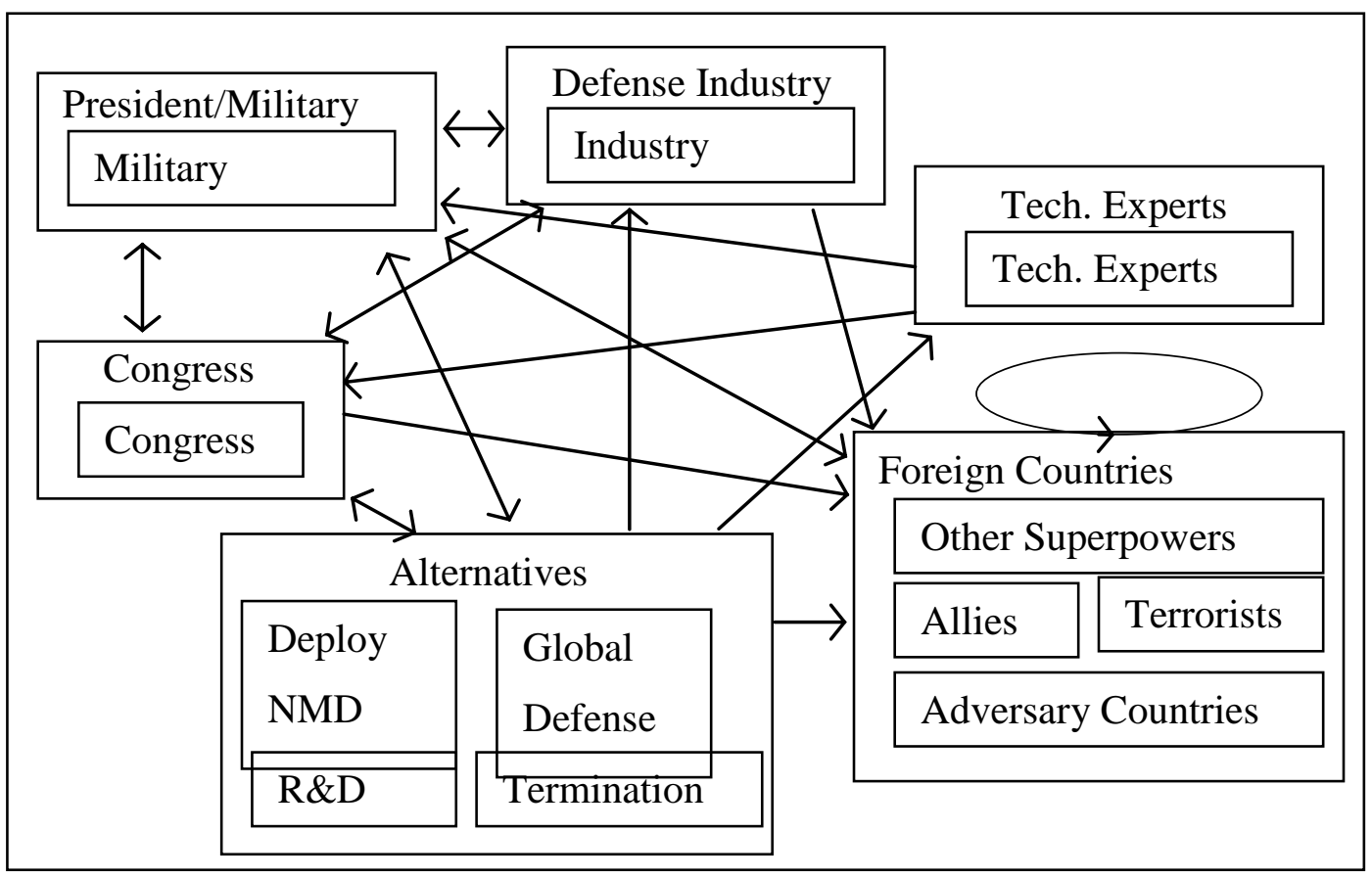

Figure 20 Decision Network under the Arms Sales

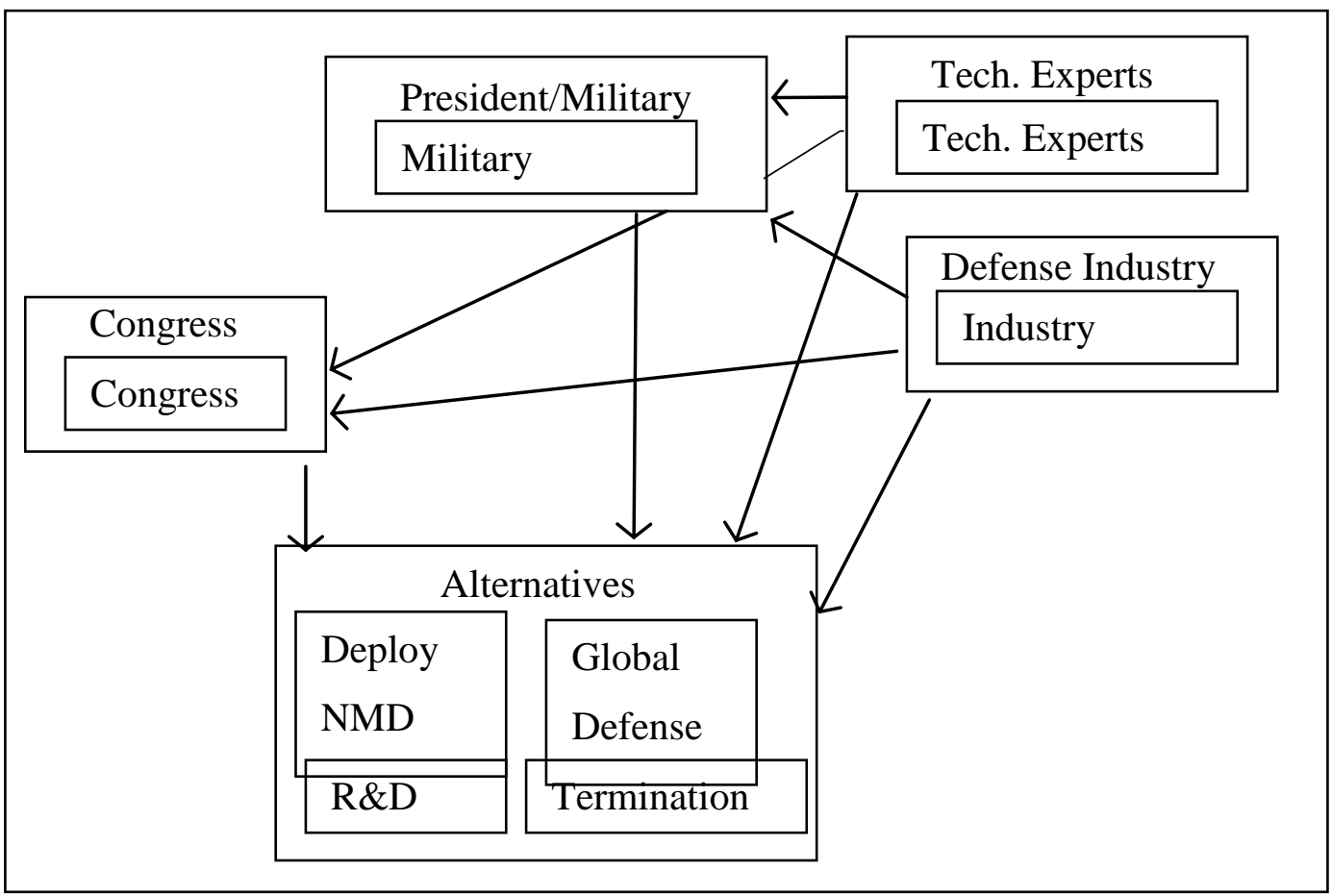

Figure 21 Decision Network under the Spin-Off 


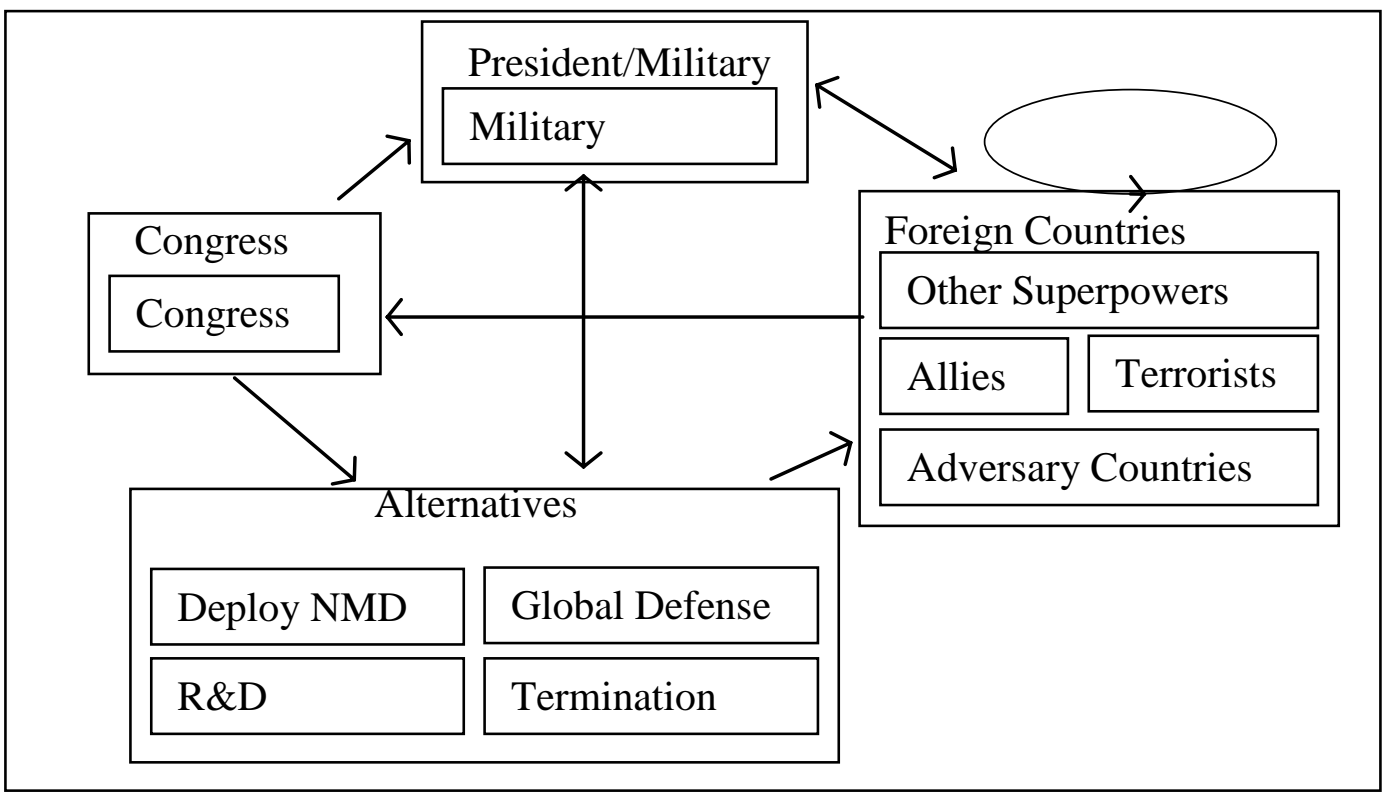

Figure 22 Decision Network under the Security Threat Control

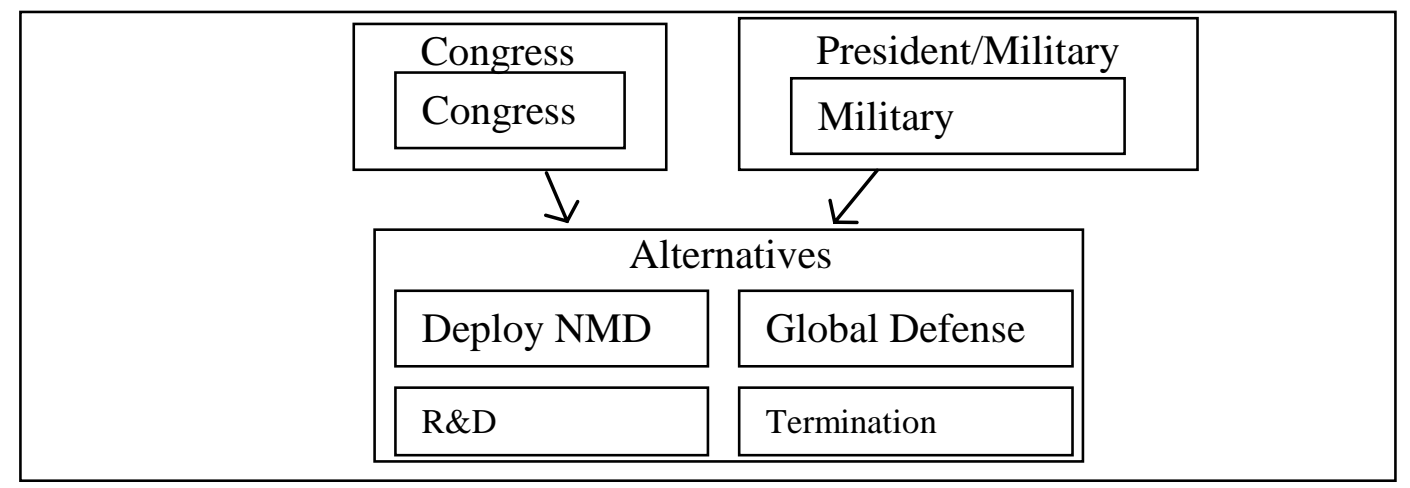

Figure 23 Decision Network under the Sunk Cost 


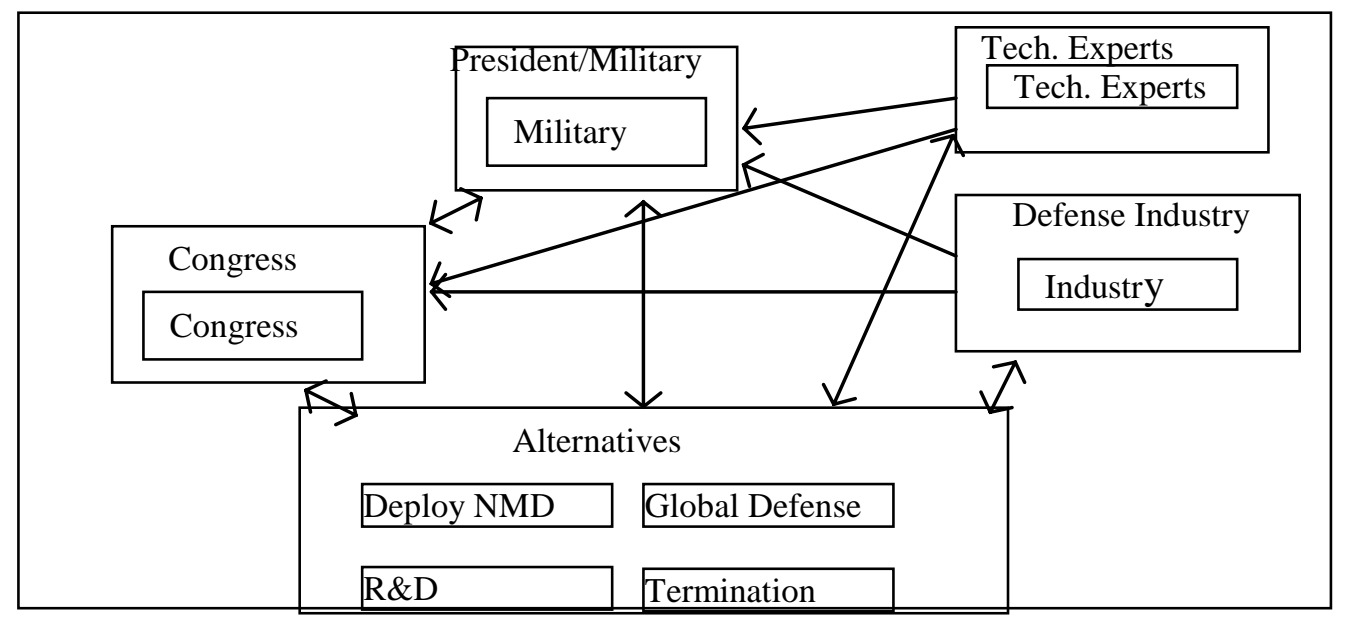

Figure 24 Decision Network under the Further Investment

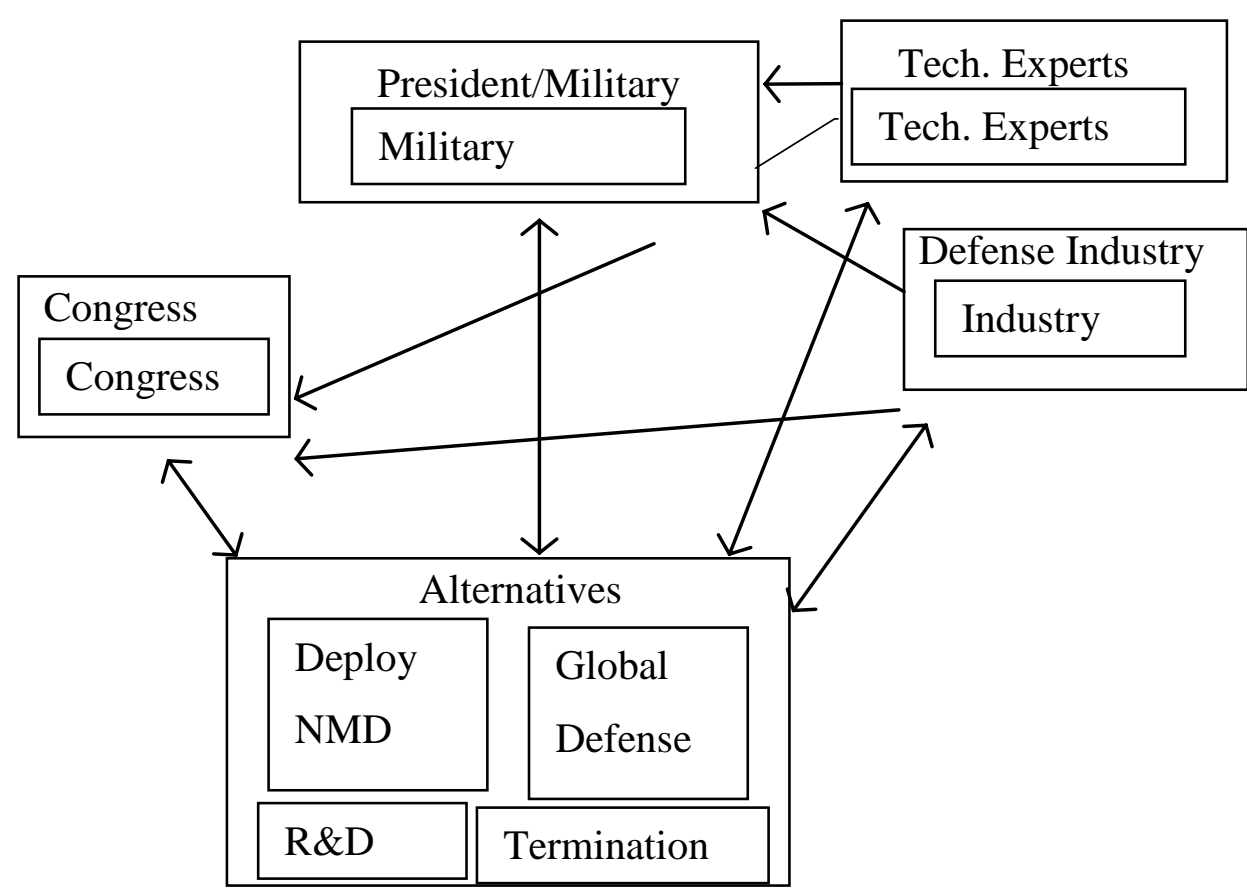

Figure 25 Decision Networks Under the Technical Feasibility 


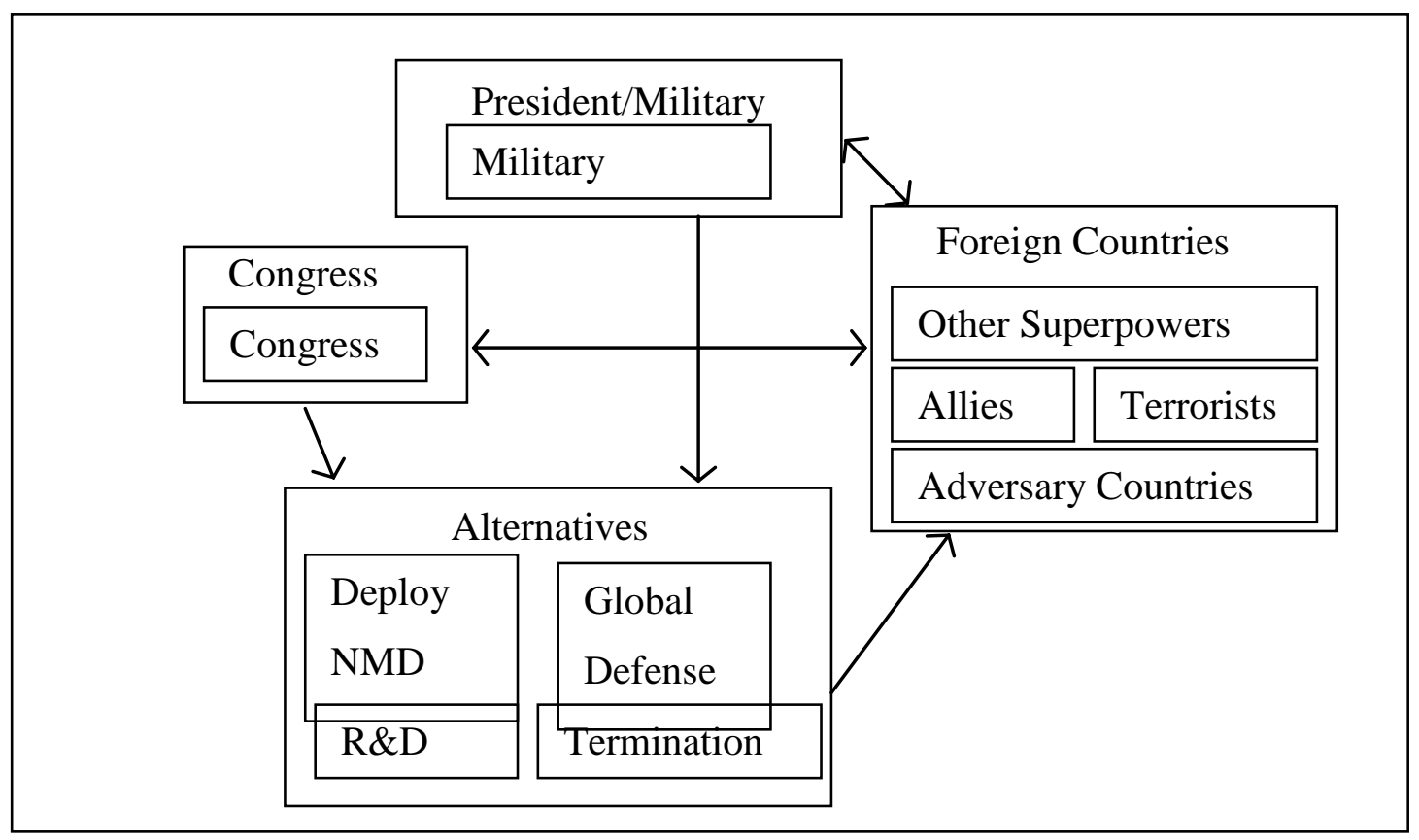

Figure26 Decision Network under the Arms Race

\section{Full DeVelopment of the AnAlysis With ReSPECT to A Single Criterion}

We explain in outline form our thinking about the network under one of the criteria. We have chosen Military Capability, one of the main control subcriteria, to elaborate the details of its decision network. There are five main parties involved in the decision making process of NMD: Congress, President/Military, Foreign Countries, Technical Experts and the Defense Industry. The latter two influence Congress and President/Military by providing their professional expertise and technical information. Allies among Foreign Countries can have a partial influence on Global Defense among the four alternatives through economic and technological cooperation.

The first block of four rows and four columns in Table 35, The Unweighted Supermatrix, indicates that Deploy NMD (NMD) and R\&D (R\&D) are influenced by Global Defense (Glob ) with priorities of 0.5760 and 0.4240 respectively. The next five columns and first four rows of Table 35, The Unweighted Supermatrix, summarize the different views of actors on the contribution of each of the four alternatives to U.S. military capability. Congress, President/Military, Defense Industry, and Technical Experts all have a say as to what extent the decision contributes to the Military Capability of the U.S. All domestic actors think that Deploy NMD will increase military capability followed by Global Defense, R\&D and Termination (Term ) but to different degrees. Deploy NMD (0.5587) was given the highest priority by Defense Industry, followed by the priority given by President/Military (0.5158), and Congress (0.5060). The lowest priority given to NMD is by Technical Experts (0.2878). It reflects the opinion of scientists who think Deploy NMD is technically infeasible and would not contribute to 
the enhancement of U.S. military capability. Only Global Defense is influenced by Allies and thus the priority of Global Defense is equal to 1.0000 .

The fifth to the last row of Table 35 shows connections among components (clusters) each consisting of a single element except for the component of Alternatives that has four elements. The priorities of the entries in these rows must be either 1.0000 or 0.0000 depending on whether there is influence among them. For example, the fifth to the ninth entries of column one have unit entries obtained from answering the question "Is the component of Congress influenced by Deploy NMD?," "Is the component of Defense Industry influenced by Deploy NMD?" and similarly for the other three alternatives. All actors are influenced by the three alternatives of Deploy NMD, Global Defense and R\&D. Note that an entire column under Termination in the Unweighted Supermatrix of Table 34 consists of zeros because nothing is influenced by Termination and that leads to dropping the entire matter of missile defense. It is worth noting that under the Security Threat criterion of Costs (not shown here), the column under Termination in the Unweighted Supermatrix consists of non-zero values because security threat to the U.S. would continue particularly if Termination is chosen as it accentuates vulnerability of U.S. security.

Table 36 shows the pairwise comparisons of the components. The judgments were obtained by answering the question "Which of two components is influenced more by a third component with respect to military capability?" The eigenvectors of the pairwise comparisons of the components in the matrices of Table 36 are exhibited in Table 37, augmented by zeros in those positions where the components on the left are not influenced by the component on top of the column. The Weighted Supermatrix of Table 38 illustrates the weighting of the blocks of the supermatrix by the priorities from the corresponding eigenvector of comparisons of the components in Table 36. Table 39, The Limit Supermatrix, yields the stable priorities of all the elements. From it, the priorities of the four alternatives are extracted and normalized. We obtain for (Deploy NMD, Global Defense, R\&D, and Termination) the corresponding values $(0.1532,0.0968,0.0438$, $0.0201)$ which when normalized by dividing by their sum yields the priority vector $(0.488,0.308$, 0.140 , and 0.064 . Similar computations are done for the remaining eight high priority criteria. An entry in each subcolumn of the supermatrix indicates the relative priority within the block to which that subcolumn belongs that an element on the left is influence by the element on top of the column with respect to Military Capability. Each subcolumn is an eigenvector imported from a corresponding pairwise comparisons matrix not shown here because its elements can be approximately formed from the ratios of the corresponding priority vector. A subcolumn of zeros indicates no influence and therefore no comparisons matrix is needed. 


\section{All Matrices for the Military Capability Decision Network of Benefits}

Table 35 The Unweighted Supermatrix

\begin{tabular}{|c|c|c|c|c|c|c|c|c|c|c|}
\hline \multirow{2}{*}{\multicolumn{2}{|c|}{$\begin{array}{l}\text { MilCap } \\
\text { Unweighted }\end{array}$}} & \multicolumn{4}{|c|}{ Altern } & \multirow{2}{*}{\begin{tabular}{|l|} 
Cong $~$ \\
Cong $~$ \\
\end{tabular}} & \multirow{2}{*}{\begin{tabular}{|l} 
Def. Ind \\
Industry
\end{tabular}} & \multirow{2}{*}{$\begin{array}{l}\text { For } \\
\text { Allies }\end{array}$} & \multirow{2}{*}{\begin{tabular}{|c|} 
Pre/Mil \\
$\sim$ \\
Militar \\
$\mathrm{y}$ \\
\end{tabular}} & \multirow{2}{*}{\begin{tabular}{|l|} 
Tech \\
Tech $\sim$
\end{tabular}} \\
\hline & & $\begin{array}{c}\mathrm{NM} \\
\mathrm{D}\end{array}$ & Glob & $\begin{array}{c}\mathrm{R} \& \\
\mathrm{D}\end{array}$ & Term & & & & & \\
\hline \multirow{8}{*}{ Altern } & \multirow{6}{*}{$\begin{array}{l}\text { NMD } \\
\text { Glob } \\
\text { R \& D }\end{array}$} & 0.000 & & 0.000 & & & & & & \multirow[b]{2}{*}{0.2878} \\
\hline & & 0 & 0.5760 & 0 & 0.0000 & 0.5060 & 0.5587 & 0.0000 & 0.5158 & \\
\hline & & 0.000 & & 0.000 & & & & & & \\
\hline & & 0 & 0.0000 & 0 & 0.0000 & 0.2890 & 0.2574 & 1.0000 & 0.2929 & 0.262 \\
\hline & & 0.000 & & 0.000 & & & & & & \\
\hline & & 0 & 0.4240 & 0 & 0.0000 & 0.1307 & 0.1382 & 0.0000 & 0.1367 & 0.269 \\
\hline & Term & 0.000 & & 0.000 & & & & & 16 & \\
\hline & \multirow{3}{*}{ Cong } & & 0.0000 & 0 & 0.0000 & 0.0744 & & 0.0000 & 0.0546 & 0.2130 \\
\hline \multirow{2}{*}{ Cong } & & 1.000 & & 1.000 & & & & & & \\
\hline & & 0 & 1.0000 & 0 & 0.0000 & 0.0000 & 1.0000 & 1.0000 & 1.0000 & 1.0000 \\
\hline \multirow{2}{*}{$\begin{array}{c}\text { Defense } \\
\text { Ind }\end{array}$} & \multirow{2}{*}{$\begin{array}{c}\text { Industr } \\
\mathrm{y}\end{array}$} & 1.000 & & 1.000 & & \multirow[b]{2}{*}{0.0000} & \multirow[b]{2}{*}{0.0000} & \multirow[b]{2}{*}{0.0000} & \multirow[b]{2}{*}{0.0000} & \multirow[b]{2}{*}{0.0000} \\
\hline & & 0 & 1.0000 & 0 & 0.0000 & & & & & \\
\hline \multirow{2}{*}{ For } & \multirow{2}{*}{ Allies } & 1.000 & & 1.000 & & & & & & \\
\hline & & 0 & 1.0000 & 0 & 0.0000 & 0.0000 & 0.0000 & 0.0000 & 1.0000 & 0.0000 \\
\hline \multirow{2}{*}{ Pre/Mil } & \multirow{2}{*}{$\begin{array}{c}\text { Militar } \\
\mathrm{y}\end{array}$} & 1.000 & & 1.000 & & \multirow[b]{2}{*}{1.0000} & \multirow[b]{2}{*}{1.0000} & \multirow[b]{2}{*}{1.0000} & \multirow[b]{2}{*}{0.0000} & \multirow[b]{2}{*}{1.0000} \\
\hline & & 0 & 1.0000 & 0 & 0.0000 & & & & & \\
\hline \multirow{2}{*}{ Tech } & \multirow{2}{*}{ Tech } & 1.000 & & 1.000 & & \multirow[b]{2}{*}{0.0000} & \multirow[b]{2}{*}{0.0000} & \multirow[b]{2}{*}{0.0000} & & \\
\hline & & 0 & 1.0000 & 0 & 0.0000 & & & & 0.0000 & 0.0000 \\
\hline
\end{tabular}

Table 36 Pairwise Comparisons Matrices and Priorities of Components

Pairwise comparing components with respect to the Alternatives component

Q: Which of a pair of components is influenced more by the Alternatives component with respect

to Military Capability?

\begin{tabular}{|c|c|c|c|c|c|c|c|}
\hline & Altern $~$ & Cong & Def. Ind $~$ & For $~$ & Pres $\sim$ & Tech & Prior. \\
\hline Altern $~$ & 1.0000 & 0.1667 & 0.2500 & 1.3300 & 0.1429 & 0.5556 & $\mathbf{0 . 0 4 8 6}$ \\
\hline Cong & 5.9999 & 1.0000 & 2.2000 & 6.2000 & 0.7407 & 3.2000 & $\mathbf{0 . 2 8 8 9}$ \\
\hline Def. Ind $\sim$ & 4.0000 & 0.4546 & 1.0000 & 4.0000 & 0.4115 & 2.2600 & $\mathbf{0 . 1 6 5 3}$ \\
\hline For $\sim$ & 0.7519 & 0.1613 & 0.2500 & 1.0000 & 0.1250 & 0.5263 & $\mathbf{0 . 0 4 2 5}$ \\
\hline Pres $\sim$ & 7.0000 & 1.3500 & 2.4300 & 8.0000 & 1.0000 & 5.1000 & $\mathbf{0 . 3 7 4 2}$ \\
\hline Tech & 1.8000 & 0.3125 & 0.4425 & 1.9000 & 0.1961 & 1.0000 & $\mathbf{0 . 0 8 0 5}$ \\
\hline
\end{tabular}

Pairwise comparing components with respect to the Congress component

Q: Which of a pair of components is influenced more by the Congress component with respect to Military Capability?
Pairwise comparing components with respect to the Defense Industry component

Q: Which of a pair of components is influenced more by the Defense Industry component with respect to Military Capability? 


\begin{tabular}{|c|c|c|c|}
\hline & Altern & Pres $\sim$ & Prior. \\
\hline Altern $\sim$ & 1.0000 & 0.5638 & $\mathbf{0 . 3 6 0 5}$ \\
\hline Pres $\sim$ & 1.7736 & 1.0000 & $\mathbf{0 . 6 3 9 5}$ \\
\hline
\end{tabular}

Pairwise comparing components with respect to the Foreign Countries component Q: Which of a pair of components is influenced more by the Foreign Countries component with respect to Military Capability?

\begin{tabular}{|c|c|c|c|c|}
\hline & Altern $~$ & Cong $\sim$ & Pres $\sim$ & Prior. \\
\hline Altern $~$ & 1.0000 & 0.6769 & 0.5388 & $\mathbf{0 . 2 2 9 2}$ \\
\hline Congr $~$ & 1.4773 & 1.0000 & 0.6600 & $\mathbf{0 . 3 1 8 1}$ \\
\hline Pres $\sim$ & 1.8561 & 1.5152 & 1.0000 & $\mathbf{0 . 4 5 2 8}$ \\
\hline
\end{tabular}

Pairwise comparing components with respect to the Presidnet/Military component

Q: Which of a pair of components is influenced more by the President/ Military component with respect to Military Capability?

\begin{tabular}{|l|l|l|l|c|}
\hline & Altern $\sim$ & Cong $\sim$ & Pres $\sim$ & $\begin{array}{c}\text { Prior } \\
.\end{array}$ \\
\hline Altern $~$ & 1.0000 & 0.5556 & 0.3259 & $\begin{array}{c}\boldsymbol{0 . 1 6 7} \\
\boldsymbol{1}\end{array}$ \\
\hline Congr $~$ & 1.8000 & 1.0000 & 0.4632 & $\begin{array}{c}\boldsymbol{0 . 2 7 8} \\
\mathbf{I}\end{array}$ \\
\hline Pres $\sim$ & 3.0682 & 2.1591 & 1.0000 & $\begin{array}{c}\boldsymbol{0 . 5 5 4} \\
\mathbf{8}\end{array}$ \\
\hline
\end{tabular}

\begin{tabular}{|l|l|l|l|l|}
\hline & Altern $~$ & Cong $\sim$ & For $~$ & Prior. \\
\hline Altern $~$ & 1.0000 & 2.1887 & 3.6604 & $\mathbf{0 . 5 7 3 5}$ \\
\hline Congr $\sim$ & 0.4569 & 1.0000 & 2.0377 & $\mathbf{0 . 2 7 9 9}$ \\
\hline For $\sim$ & 0.2732 & 0.4907 & 1.0000 & $\mathbf{0 . 1 4 6 7}$ \\
\hline
\end{tabular}

Pairwise comparing components with respect to the Technical

Experts component

Q: Which of a pair of components is influenced more by the

Technical Experts component with respect to Military Capability?

\begin{tabular}{|l|c|c|c|c|}
\hline & Altern $\sim$ & Cong $\sim$ & Pres $\sim$ & $\begin{array}{c}\text { Prior } \\
\text {. }\end{array}$ \\
\hline Altern $~$ & 1.0000 & 2.5379 & 2.5379 & $\begin{array}{c}\boldsymbol{0 . 5 5 9} \\
\mathbf{3}\end{array}$ \\
\hline Congr & 0.3940 & 1.0000 & 1.0000 & $\begin{array}{c}\boldsymbol{0 . 2 2 0} \\
\mathbf{4}\end{array}$ \\
\hline Pres $\sim$ & 0.3940 & 1.0000 & 1.0000 & $\begin{array}{c}\mathbf{0 . 2 2 0} \\
\mathbf{4}\end{array}$ \\
\hline
\end{tabular}


Table 37 Priorities Matrix of Eigenvectors

How much components are influenced by each component; imported from the matrices of Table 36 above

\begin{tabular}{|c|c|c|c|c|c|c|}
\hline Clusters & Altern & Cong $\sim$ & $\begin{array}{c}\text { Def. } \\
\text { Ind } ~\end{array}$ & For & Pres & Tech $~$ \\
\hline Altern $~$ & 0.0486 & 0.3605 & 0.2292 & 0.1671 & 0.5735 & 0.5593 \\
\hline Cong $\sim$ & 0.2889 & 0.0000 & 0.3181 & 0.2781 & 0.2799 & 0.2204 \\
\hline Def. Ind $\sim$ & 0.1653 & 0.0000 & 0.0000 & 0.0000 & 0.0000 & 0.0000 \\
\hline For $~$ & 0.0425 & 0.0000 & 0.0000 & 0.0000 & 0.1467 & 0.0000 \\
\hline Pres $\sim$ & 0.3742 & 0.6395 & 0.4528 & 0.5548 & 0.0000 & 0.2204 \\
\hline Tech $~$ & 0.0805 & 0.0000 & 0.0000 & 0.0000 & 0.0000 & 0.0000 \\
\hline
\end{tabular}

Table 38 The Weighted Supermatrix

Priorities from Table 37 are used to weight corresponding blocks of unweighted supermatrix of

Table 35

\begin{tabular}{|c|c|c|c|c|c|c|c|c|}
\hline \multirow{2}{*}{$\begin{array}{c}\text { MilCap } \\
\text { Weighted }\end{array}$} & & \multicolumn{2}{|r|}{ Altern } & Cong & \multirow{2}{*}{$\begin{array}{c}\text { Def. } \\
\text { Ind }\end{array}$} & \multirow{2}{*}{\begin{tabular}{|l|} 
For \\
Allies \\
\end{tabular}} & \multirow{2}{*}{\begin{tabular}{|l} 
Pre/Mil \\
Military \\
\end{tabular}} & \multirow{2}{*}{\begin{tabular}{|l} 
Tech \\
Tech \\
\end{tabular}} \\
\hline & & NMD & lob R \& D Term & COne & & & & \\
\hline Altern & NMD & 0.000 & 02800.00000 .000 & 0.1824 & 0.1280 & 0.0000 & 0.2958 & 0.16 \\
\hline & G & & & & 0.0590 & 0.1671 & 580 & 0.146 \\
\hline & $\mathrm{D}$ & $0 .($ & & & 0.0317 & 0.0000 & 0.0784 & 0.132 \\
\hline & & & & & 0.0105 & 00 & 13 & 10 \\
\hline Cong & Cong & 0.30 & 370.0000 & 00 & 0.3181 & 0.2780 & 0.2799 & 0.220 \\
\hline efense Inc & Indu & 0.17 & 0.16530 .17370 .00 & 00 & 0.0000 & 0.0000 & 00 & 0.000 \\
\hline For & Allies & 0.0 & 00 & 00 & 0.0000 & 0.0000 & 467 & 0.000 \\
\hline Pre/Mil & Military & 0.3 & 0.0000 & 95 & 0.4528 & 0.5548 & 0.0000 & 0.220 \\
\hline Tech & Tech & 0.08 & 0.08050 .08460 .0000 & 0.0000 & 0.0000 & 0.0000 & 0.0000 & 0.000 \\
\hline
\end{tabular}

Table 39 The Limit Supermatrix

The weighted supermatrix raised to sufficiently large powers to stabilize within rounded off four place decimals

\begin{tabular}{|c|c|c|c|c|c|c|c|}
\hline \multirow{2}{*}{$\begin{array}{l}\text { MilCap } \\
\text { Limited }\end{array}$} & & Altern & \multirow{2}{*}{\begin{tabular}{|l|} 
Cong $~$ \\
Cong
\end{tabular}} & \multirow{2}{*}{$\begin{array}{c}\text { Def. } \\
\text { Ind } ~ \\
\text { Industry }\end{array}$} & \multirow{2}{*}{\begin{tabular}{|l|} 
For \\
Allies \\
\end{tabular}} & \multirow{2}{*}{\begin{tabular}{|l} 
Pre/Mil \\
Military
\end{tabular}} & \multirow{2}{*}{\begin{tabular}{|l} 
Tech \\
Tech
\end{tabular}} \\
\hline & & NMD Glob R \& D Term & & & & & \\
\hline Altern & NMD & $\begin{array}{lllll}0.1532 & 0.1532 & 0.1532 & 0.0000\end{array}$ & 0.1532 & 0.1532 & 0.1532 & 0.1532 & 0.1532 \\
\hline & Glob & $\begin{array}{llll}0.0968 & 0.0968 & 0.0968 & 0.0000\end{array}$ & 0.0968 & 0.0968 & 0.0968 & 0.0968 & 0.0968 \\
\hline & $R \& D$ & $\begin{array}{llll}0.0438 & 0.0438 & 0.00\end{array}$ & 0.0438 & 0.0438 & 0.0438 & 0.0438 & 0.0438 \\
\hline & Term & $\begin{array}{llll}0.0201 & 0.0201 & 0.0201 & 0.0000\end{array}$ & 0.0201 & 0.0201 & 0.0201 & 0.0201 & 0.0201 \\
\hline Cong & Cong & 0.22240 .22240 .22240 .0000 & 0.2224 & 0.2224 & 0.2224 & 0.2224 & 0.2224 \\
\hline Defense Ind- & Industry & 0.05130 .05130 .05130 .0000 & 0.0513 & 0.0513 & 0.0513 & 0.0513 & 0.0513 \\
\hline For & Allies & $\begin{array}{llll}0.0619 & 0.0619 & 0.0619 & 0.0000\end{array}$ & 0.0619 & 0.0619 & 0.0619 & 0.0619 & 0.0619 \\
\hline Pre/Mil & Military & $\begin{array}{lllll}0.3255 & 0.3255 & 0.3255 & 0.0000\end{array}$ & 0.3255 & 0.3255 & 0.3255 & 0.3255 & 0.3255 \\
\hline Tech & Tech & $\begin{array}{lllll}0.0250 & 0.0250 & 0.0250 & 0.0000\end{array}$ & 0.0250 & 0.0250 & 0.0250 & 0.0250 & 0.0250 \\
\hline
\end{tabular}




\section{BOCR WEIGHT DEVELOPMENT}

The judgments used in this analysis were our interpretation of what experts thought about the various issues obtained from the vast reading of the literature we examined and from following the news closely for a period of more than six months. We also consulted some knowledgeable people on the subject in the area. We quickly realized there is no single expert in all the criteria we considered. Sensitivity analysis given later would essentially vary these judgments widely to determine the stability of the outcome. The assessment criteria used to determine the priorities of the BOCR merits are shown in

Figure 26. These are World Peace, Human Well-being, and International Politics. All these criteria have subcriteria under them. The three subcriteria, Adversary Countries, Security Dilemma and Terrorism cover all the causes disturbing or stabilizing peace in the world. The first subcriterion, Adversary Countries, concerns the potential threats by adversary countries. The second criterion, Security Dilemma, means that increasing one country's security inevitably decreases other countries' security. Terrorism indicates any possibility of the rise or decline of terrorism in the world. Human Well-being includes Technological Advancement and Market Creation. Technological Advancement driven by the NMD research and development process can ultimately benefit all people, particularly in providing possible space exploration that can lead to the creation of new markets. Moreover, the 21 st century is characterized as a postindustrialization era. Service industries in communication and transportation will benefit not only businesses associated with these industries, but also consumers who can enjoy the products from the new market. The last criterion is International Politics. It is composed of two subcriteria, Military Relations and Diplomatic Relations. Military Relations refer to the impact of NMD on relations with U.S. allies for better or for worse. Also, the impact of NMD on diplomatic relations among all countries should be considered. The priorities shown next to the criteria and subcriteria in

Figure were obtained through the usual pairwise comparison process of the AHP according to their importance with respect to their higher-level goal or parent criterion.

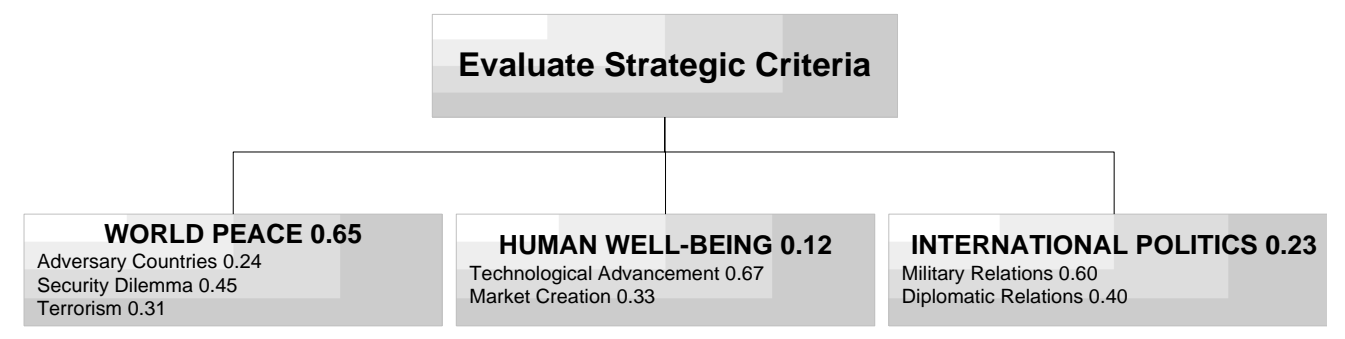

Figure 26 Strategic Criteria for BOCR Ratings

The four merits of BOCR were rated according to the five intensities listed in Table 440. The priorities of the intensities shown in the table were derived from pairwise comparisons. The weighted values of the subcriteria shown in Table 40, are multiplied times the value of the assigned intensity and summed for each column to yield the outcome, the BOCR values at the bottom of the table. 


\begin{tabular}{|c|c|c|c|c|c|}
\hline \multicolumn{6}{|c|}{ Very High (0.419), High (0.263), Medium (0.160), Low (0.097), Very Low (0.061) } \\
\hline Criteria & Subcriteria & Benefits & Opportunities & Costs & Risks \\
\hline \multirow[t]{3}{*}{$\begin{array}{l}\text { World } \\
\text { Peace }\end{array}$} & $\begin{array}{l}\text { Adversary } \\
\text { Countries }(0.156)\end{array}$ & $\begin{array}{l}\text { Very } \\
\text { High }\end{array}$ & Medium & High & Very Low \\
\hline & $\begin{array}{l}\text { Security Dilemma } \\
(0.293)\end{array}$ & $\begin{array}{l}\text { Very } \\
\text { Low }\end{array}$ & Very Low & Very High & Very Low \\
\hline & Terrorism $(0.202)$ & Medium & Very Low & High & High \\
\hline \multirow[t]{2}{*}{$\begin{array}{l}\text { Human } \\
\text { Well- } \\
\text { Being }\end{array}$} & $\begin{array}{l}\text { Technological } \\
\text { Advancement } \\
(0.080)\end{array}$ & High & High & Low & Very Low \\
\hline & $\begin{array}{l}\text { Market Creation } \\
(0.040)\end{array}$ & Medium & High & Very Low & Very Low \\
\hline \multirow[t]{2}{*}{$\begin{array}{l}\text { Internation } \\
\text { al Politics }\end{array}$} & $\begin{array}{l}\text { Military Relations } \\
(0.138)\end{array}$ & High & High & Medium & Very Low \\
\hline & $\begin{array}{l}\text { Diplomatic } \\
\text { Relations (0.092) }\end{array}$ & Low & Low & Low & Very High \\
\hline Priorities & & 0.264 & 0.185 & 0.361 & 0.190 \\
\hline
\end{tabular}

Note that BOCR are rated one at a time and are not obtained from paired comparisons. They are obtained using the rating approach of the AHP.

As we said earlier if we weight the priorities derived in Table shown in boldface by the corresponding priorities of the merits just derived in Table 4Table 40 above and then add we get:

$$
\begin{aligned}
.264 x .284+264 \times .240+ & .185 x .520+.185 x .326+.361 \times .687+.361 x .12+.361 x .105 \\
& +.190 x .430+.190 x .268 \approx .76 .
\end{aligned}
$$

In most of our studies we attempt to use enough control criteria factors under any given merit so that they total not less than $70 \%$.

Table 41 shows the idealized priorities of the four alternatives (developed from their values in the corresponding limit supermatrix) with respect to the control criteria of the merits and also the synthesis with respect to the criteria of the alternatives for each merit to obtain the final outcome for that merit. 
Table 41 Synthesis of the Alternatives for each BOCR Merit

\begin{tabular}{|c|c|c|c|c|}
\hline Merit & $\begin{array}{l}\text { Alter- } \\
\text { natives }\end{array}$ & \multicolumn{2}{|c|}{$\begin{array}{l}\text { Idealized Results under the control criteria of } \\
\text { each merit. } \\
\text { (The values are obtained by normalizing the } \\
\text { selected control criteria in the Goal Column } \\
\text { in the Limiting Matrix.) }\end{array}$} & $\begin{array}{l}\text { Multiply Idealized vectors } \\
\text { in previous column by CC } \\
\text { priorities and sum to } \\
\text { obtain composite vector }\end{array}$ \\
\hline BENEFITS & $\begin{array}{l}\text { A1 } \\
\text { A2 } \\
\text { A3 } \\
\text { A4 }\end{array}$ & $\begin{array}{c}\text { Military } \\
\text { Capability } \\
(.542) \\
1.000 \\
.62 \\
.282 \\
.129\end{array}$ & $\begin{array}{c}\text { Tech Ad-vancement } \\
(.458) \\
.928 \\
1.000 \\
.448 \\
.085\end{array}$ & $\begin{array}{l}0.967 \\
0.796 \\
0.358 \\
0.109\end{array}$ \\
\hline $\begin{array}{l}\text { OPPOR- } \\
\text { TUNITIES }\end{array}$ & $\begin{array}{l}\text { A1 } \\
\text { A2 } \\
\text { A3 } \\
\text { A4 }\end{array}$ & $\begin{array}{l}\text { Arms } \\
\text { Sales } \\
(.614) \\
1.000 \\
.674 \\
.341 \\
.190\end{array}$ & 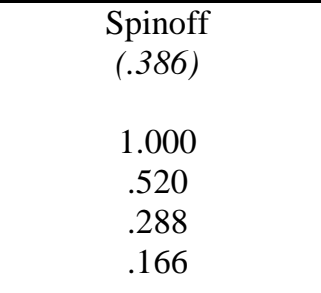 & $\begin{array}{l}1.000 \\
0.615 \\
0.321 \\
0.181\end{array}$ \\
\hline Costs & $\begin{array}{l}\text { A1 } \\
\text { A2 } \\
\text { A3 } \\
\text { A4 }\end{array}$ & $\begin{array}{c}\text { Security } \\
\begin{array}{c}(.687) \\
.183 \\
.344 \\
.579 \\
1.000\end{array}\end{array}$ & $\begin{array}{c}\text { Further } \\
\text { Investmt } \\
(.105) \\
1.000 \\
.496 \\
.279 \\
.147\end{array}$ & $\begin{array}{l}.386 \\
.393 \\
.512 \\
.794\end{array}$ \\
\hline RISKS & $\begin{array}{l}\text { A1 } \\
\text { A2 } \\
\text { A } 3 \\
\text { A4 }\end{array}$ & $\begin{array}{c}\text { Technical } \\
\text { Failure } \\
(.430) \\
1.000 \\
.621 \\
.375 \\
.262\end{array}$ & $\begin{array}{l}\text { Arms } \\
\text { Race } \\
(.268) \\
1.000 \\
.693 \\
.441 \\
.302\end{array}$ & $\begin{array}{l}1.000 \\
.648 \\
.401 \\
.277\end{array}$ \\
\hline
\end{tabular}

The merit priorities are represented in the formulas in Table 42 by $\mathbf{b}, \mathbf{o}, \mathbf{c}$ and $\mathbf{r}$ respectively and imported from Table 40, while the priorities of the alternatives imported from the right column of Table 41 are represented by $\mathbf{B}, \mathbf{O}, \mathbf{C}$ and $\mathbf{R}$.

Table 42 Overall Synthesis of the Alternatives 


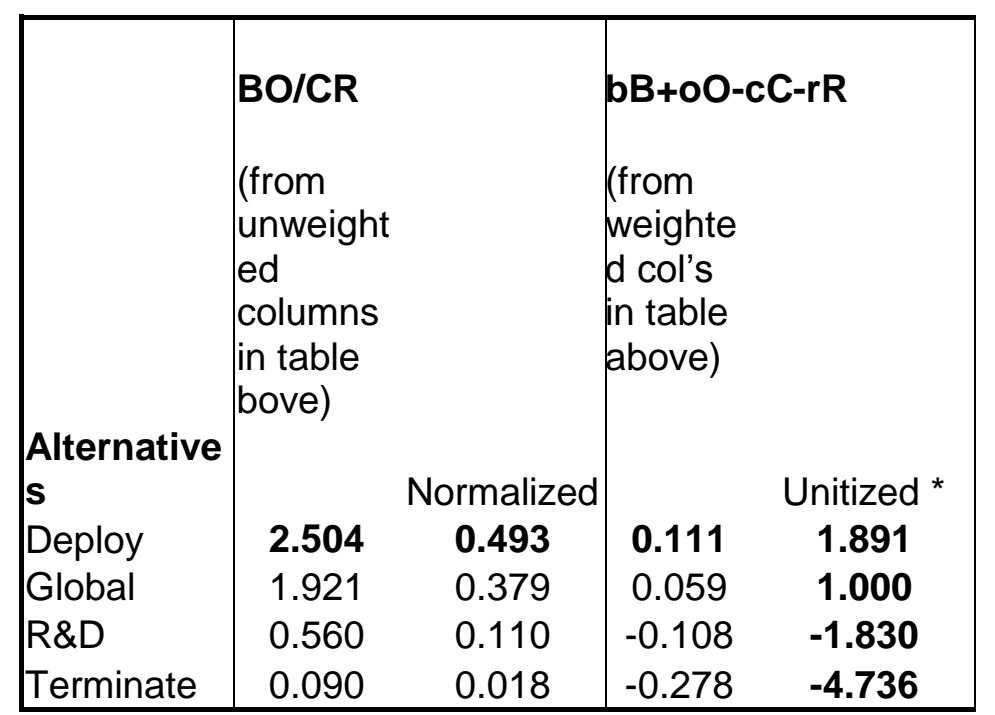

*Unitized means to divide each number in the column by the number with the smallest absolute value

Here we see that all three formulas give the same outcome to deploy as the best alternative. The conclusion of this analysis is that pursuing the deployment of NMD is the best alternative. But we must now examine how realistic this outcome is.

One might have different judgments in comparing the importance of BOCR or of the nine control criteria. To ensure the stability of the outcome of our analysis, we conducted sensitivity analysis.

\section{A. SEnSitivity ANAlysis AT THE BOCR LEVEL}

First, we increased and decreased one of the four merits of BOCR keeping the others proportionally the same. For example, if benefits were to be increased from its original priority 0.264 to 0.500 , the sum of the other three merits would comprise the other 0.500 and the proportion among them would remain the same as before and their new priorities would be: opportunities, 0.124, costs, 0.246, and risks, 0.130. We found that no matter how much we increased or decreased the priorities of benefits, opportunities and costs the overall ranks of the final outcome were preserved although these experiments changed the magnitude of the superiority of the best alternative, Deploy NMD (for example, from 0.301 to 0.431 for benefits as Figure 27 shows). Only changing the priority of risks reversed the ranks of the four alternatives as shown in Figure 27. This occurred only when the priority of the risks was 0.375 or more. Then, Termination gradually became third then second and finally the best alternative as the priority of risks was increased more and more. 


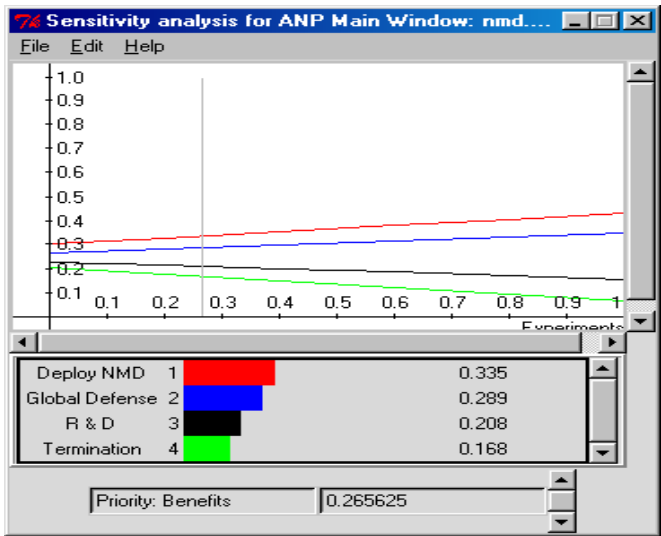

Sensitivity Analysis for Benefits

The rank remains the same regardless of the priorities of benefits

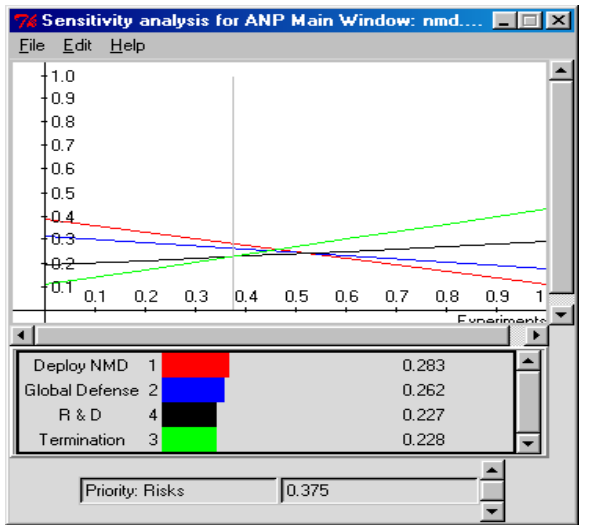

Sensitivity Analysis for Risks

Termination becomes the more preferred alternative as the priority of risks increases
Figure

27

Sensitiv

ity

Analysi

$\mathrm{s}$ for

Benefit

$\mathrm{s}$ and

for

Risks

B.

SENSIT

IVITY

ANALY

SIS AT

THE

CONTR

\section{ol Criteria Level}

We did similar tests for the nine criteria that have decision networks. We found that the outcome was very stable and did not change the overall ranks except for changes in the importance of the three criteria: Security Threat, Sunk Cost and Further Investment all under costs. When the priority of Security Threat decreased to about 0.172 from 0.21 or the priority of Sunk Cost increased to 0.753 or the priority of Further Investment increased to 0.734 Figures 28 and 29 Termination gradually began to move to third, second and finally to first rank position.

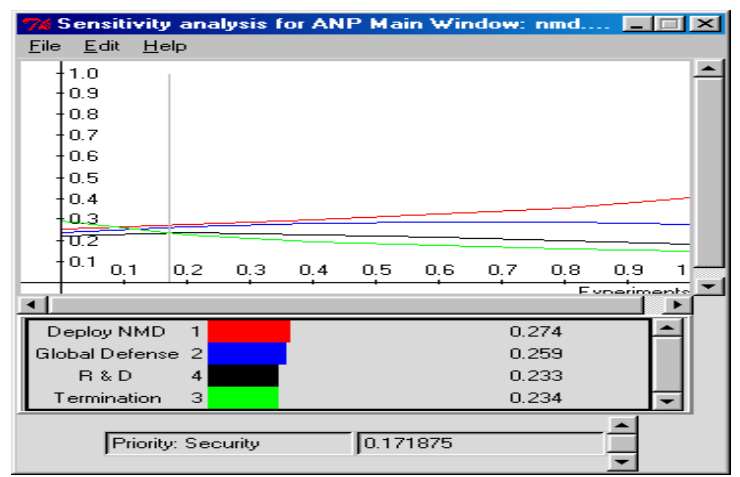

Figure 28 Sensitivity Analysis for Security Threat

If the priority of Security Threat becomes less than about 0.172 , Termination becomes the more preferred alternative 


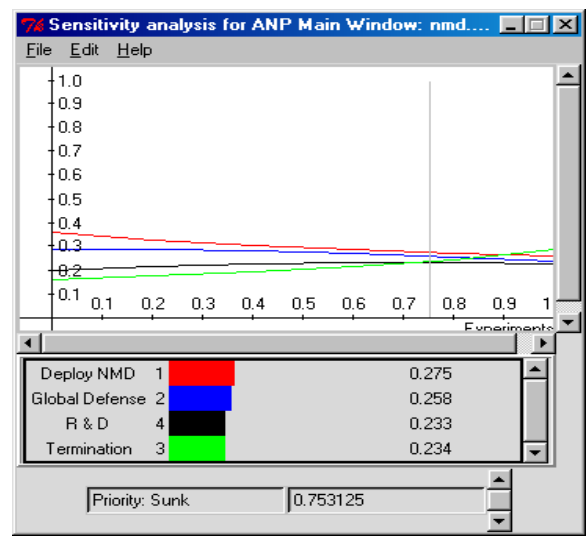

Sensitivity Analysis for Sunk Cost

If the priority of Sunk Cost becomes larger than 0.753 , Termination becomes the more preferred alternative.

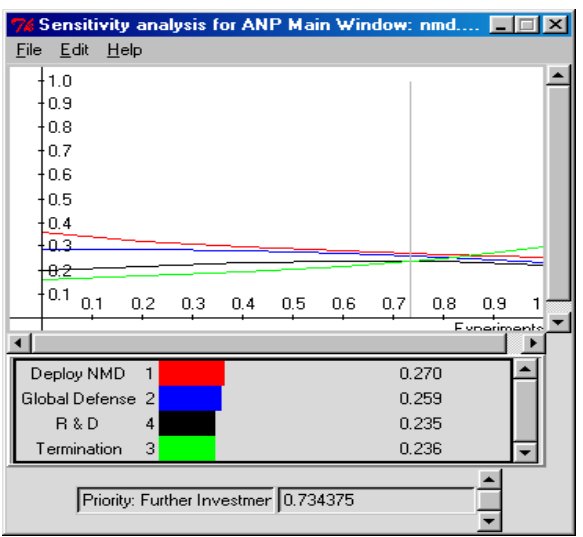

Sensitivity Analysis for Further Investment

If the priority of Further Investment becomes larger than 0.734 Termination becomes the more preferred alternative.

Figure 29 Sensitivity Analysis for Sunk Cost and Further Investment

Some are highly concerned with risks associated with NMD, such as Technical Failure and Arms Race. We did another test using larger priorities for risks to see if it would change the outcome. In that case, the control criterion, U.S. Reputation, under risks replaced the control criterion, Further Investment, under costs. Interestingly enough, the ranks of the alternatives were the same as in Table with a slightly higher priority for Deploy NMD.

Our sensitivity analysis indicates that the final ranks of the alternatives might change, but such change requires making extreme assumptions on the priorities of BOCR and of their corresponding control criteria. The outcome in Table 42 is very stable and the United States should choose Deploy NMD as the best alternative for the decision. I lectured on this on February 21, 2002 at National Defense University and from the comments I received in writing which I still have, it was very well received. It is not very surprising that the United States did make the decision to Deploy in December 2002. I am not sure what effect this analysis had on that decision if any. But the U.S. has excellent think tanks who can do a good job of analyzing a decision. It is certain that it took us a lot less time to do this analysis and no money at all than it takes a think tank in time and resources.

\section{Group Decision Making $[1,6]$}

Here we consider two issues in group decision making. The first is how to aggregate individual judgments, and the second is how to construct a group choice from individual choices. In reality group decisions should not go by consensus because not all people feel the same about things. A minority can have very strong commitments to a cause and can give rise to disruptions that the majority feels lukewarm about. There is no hiding from this issue in the real world. The reciprocal property plays an important role in combining the judgments of several individuals to obtain a judgment for a group. Judgments must be combined so that the reciprocal of the synthesized judgments must be equal to the syntheses of the reciprocals of these judgments. It has been 
proved that the geometric mean is the unique way to do that. If the individuals are experts, they my not wish to combine their judgments but only their final outcome from a hierarchy. In that case one takes the geometric mean of the final outcomes. If the individuals have different priorities of importance their judgments (final outcomes) are raised to the power of their priorities and then the geometric mean is formed.

\section{How to Aggregate Individual Judgments}

Let the function $f\left(x_{1}, \ldots, x_{n}\right)$ for synthesizing the judgments given by $n$ judges, satisfy the

(i) Separability condition $(\mathrm{S}): f\left(x_{1}, \ldots, x_{n}\right)=g\left(x_{1}\right) \ldots g\left(x_{n}\right)$, for all $x_{1}, \ldots, x_{n}$ in an interval $\mathrm{P}$ of positive numbers, where $g$ is a function mapping $\mathrm{P}$ onto a proper interval $\mathrm{J}$ and is a continuous, associative and cancellative operation. [(S) means that the influences of the individual judgments can be separated as above.]

(ii) Unanimity condition (U): $f(x, \ldots, x)=x$ for all $x$ in P. [(U) means that if all individuals give the same judgment $x$, that judgment should also be the synthesized judgment.]

(iii) Homogeneity condition ( $\mathrm{H}): \quad f\left(u x_{1}, \ldots, u x_{n}\right)=u f\left(x_{1}, \ldots, x_{n}\right)$ where $u>0$ and $x_{k}, u x_{k}$ $(k=1,2, \ldots, n)$ are all in P. [For ratio judgments $(\mathrm{H})$ means that if all individuals judge a ratio $u$ times as large as another ratio, then the synthesized judgment should also be $u$ times as large.]

(iv) Power conditions $\left(\mathrm{P}_{\mathrm{p}}\right): f\left(x_{1}^{p}, \ldots, x_{n}^{p}\right)=f^{p}\left(x_{1}, \ldots, x_{n}\right) .\left[\left(\mathrm{P}_{2}\right)\right.$ for example means that if the $k$ th individual judges the length of a side of a square to be $x_{k}$, the synthesized judgment on the area of that square will be given by the square of the synthesized judgment on the length of its side.]

Special case $\left(\mathrm{R}=\mathrm{P}_{-1}\right)$ :

$$
f\left(\frac{1}{x_{1}}, \ldots, \frac{1}{x_{n}}\right)=1 / f\left(x_{1}, \ldots, x_{n}\right) .
$$

[(R) is of particular importance in ratio judgments. It means that the synthesized value of the reciprocal of the individual judgments should be the reciprocal of the synthesized value of the original judgments.] Aczel and Saaty [1] proved the following theorem:

Theorem The general separable $(S)$ synthesizing functions satisfying the unanimity $(U)$ and homogeneity $(H)$ conditions are the geometric mean and the root-mean-power. If moreover the reciprocal property $(R)$ is assumed even for a single $n$-tuple $\left(x_{1}, \ldots, x_{n}\right)$ of the judgments of $n$ individuals, where not all $x_{k}$ are equal, then only the geometric mean satisfies all the above conditions.

In any rational consensus, those who know more should, accordingly, influence the consensus more strongly than those who are less knowledgeable. Some people are clearly wiser and more sensible in such matters than others, others may be more powerful and their opinions should be given appropriately greater weight. For such unequal importance of voters not all $g$ 's in (S) are the same function. In place of (S), the weighted separability property (WS) is now: $f\left(x_{1}, \ldots, x_{n}\right)=g_{1}\left(x_{1}\right) \ldots g_{n}\left(x_{n}\right)$ [(WS) implies that not all judging individuals have the same weight when the judgments are synthesized and the different influences are reflected in the different functions $\left(g_{1}, \ldots, g_{n}\right)$.]

In this situation, Aczel and Alsina [1] proved the following theorem:

Theorem The general weighted-separable (WS) synthesizing functions with the unanimity $(U)$ 
and homogeneity $(H)$ properties are the weighted geometric mean $f\left(x_{1}, x_{2}, \ldots, x_{n}\right)=$ $=x_{1}^{q_{1}} x_{2}^{q_{2}} \ldots x_{n}^{q_{n}}$ and the weighted root-mean- powers $f\left(x_{1}, x_{2}, \ldots, x_{n}\right)=\sqrt[\gamma]{q_{1} x_{1}^{\gamma}+q_{2} x_{2}^{\gamma} \ldots+q_{n} x_{n}^{\gamma}}$, where $q_{1}+\ldots+q_{n}=1, q_{k}>0, k=1, \ldots, n, \gamma>0$, but otherwise $q_{1}, \ldots, q_{n}, \gamma$ are arbitrary constants.

If $f$ also has the reciprocal property (R) and for a single set of entries $\left(x_{1}, \ldots, x_{n}\right)$ of judgments of $n$ individuals, where not all $x_{k}$ are equal, then only the weighted geometric mean applies. We give the following theorem which is an explicit statement of the synthesis problem that follows from the previous results, and applies to the second and third cases of the deterministic approach:

Theorem If $x_{1}^{(i)}, \ldots, x_{n}^{(i)} i=1, \ldots, m$ are rankings of $n$ alternatives by $m$ independent judges and if $a_{i}$ is the importance of judge i developed from a hierarchy for evaluating the judges, and hence

$$
\sum_{i=1}^{m} a_{i}=1, \text { then }\left(\begin{array}{c}
m \\
\Pi x_{1}^{a_{i}} \\
i=1
\end{array}\right), \ldots,\left(\begin{array}{c}
m \\
\Pi x_{n}^{a_{i}} \\
i=1
\end{array}\right)
$$

are the combined ranks of the alternatives for the $m$ judges.

The power or priority of judge $i$ is simply a replication of the judgment of that judge (as if there are as many other judges as indicated by his/her power $a_{i}$ ), which implies multiplying his/her ratio by itself $a_{i}$ times, and the result follows.

The first requires knowledge of the functions which the particular alternative performs and how well it compares with a standard or benchmark. The second requires comparison with the other alternatives to determine its importance.

\section{On the Construction of Group Choice from Individual Choices}

Given a group of individuals, a set of alternatives (with cardinality greater than 2), and individual ordinal preferences for the alternatives, Arrow proved with his Impossibility Theorem that it is impossible to derive a rational group choice (construct a social choice function that aggregates individual preferences) from ordinal preferences of the individuals that satisfy the following four conditions, i.e., at least one of them is violated:

Decisiveness: the aggregation procedure must generally produce a group order.

Unanimity: if all individuals prefer alternative $\mathrm{A}$ to alternative $\mathrm{B}$, then the aggregation procedure must produce a group order indicating that the group prefers $\mathrm{A}$ to $\mathrm{B}$.

Independence of irrelevant alternatives: given two sets of alternatives which both include A and $\mathrm{B}$, if all individuals prefer $\mathrm{A}$ to $\mathrm{B}$ in both sets, then the aggregation procedure must produce a group order indicating that the group, given any of the two sets of alternatives, prefers A to B.

No dictator: no single individual preferences determine the group order.

Using the ratio scale approach of the AHP, it can be shown that because now the individual preferences are cardinal rather than ordinal, it is possible to derive a rational group choice satisfying the above four conditions. It is possible because: a) Individual priority scales can always be derived from a set of pairwise cardinal preference judgments as long as they form at least a minimal spanning tree in the completely connected graph of the elements being compared; and b) The cardinal preference judgments associated with group choice belong to an absolute scale that represents the relative intensity of the group preferences (Saaty and Vargas 2003). 


\section{Conclusions}

The ANP is a useful way to deal with complex decisions that involve dependence and feedback analyzed in the context of benefits, opportunities, costs and risks. It has been applied literally to hundreds of examples both real and hypothetical. What is important in decision making is to produce answers that are valid in practice. The ANP has also been validated in several examples. People often argue that judgment is subjective and that one should not expect the outcome to correspond to objective data. But that puts one in the framework of garbage in garbage out without the assurance of the long term validity of the outcome. In addition, most other approaches to decision making are normative. They say, "If you are rational you do as I say." But what people imagine is best to do and what conditions their decisions face after they are made can be very far apart in the real world. That is why the framework of the ANP is descriptive as in science rather than normative and prescriptive. It produces outcomes that are best not simple according to the decision maker's values, but also to the risks and hazards faced by the decision.

It is unfortunate that there are people who use fuzzy sets without proof to alter the AHP when it is known that fuzzy applications to decision making have been ranked as the worst among all methods. Buede and Maxwell [4] write about their findings, "These experiments demonstrated that the MAVT and AHP techniques, when provided with the same decision outcome data, very often identify the same alternatives as 'best'. The other techniques are noticeably less consistent with MAVT, the Fuzzy algorithm being the least consistent." The fundamental scale used in the AHP/ANP to represent judgments is already fuzzy. To fuzzify it further does not improve the outcome as we have shown through numerous examples [5]. The intention of fuzzy seems to be to perturb the judgments in the AHP. It is already known in mathematics that perturbing the entries of a matrix perturbs the eigenvector by a small amount but not necessarily in a more valid direction. We invite the reader to examine my latest book on the matter. It is regrettable that air post often costs more than the price of the paper back book itself.

The SuperDecisions software used to analyze complex decisions is named after the supermatrix. It can be downloaded free from creativedecisions.com. and is available free on the internet along with a manual together with numerous applications to enable the reader to apply it to his/her decision. Alternatively, go to www.superdecisions.com/ saaty and download the SuperDecisions software. The installation file is the .exe file in the software folder. The serial number is located in the doc file that is in the same folder. The important thing may be not the software but the models which are in a separate folder called models. The military are constantly involved in making complex decisions and appear to like using the ANP and investing in its development. Why do we do all this with so much effort? Because we believe strongly in the creativity of the human race and hope that our world will become increasingly more rational in making its decisions and resolving its conflicts.

\section{References}

[1] Saaty, T. L. (2006). Fundamentals of the Analytic Hierarchy Process, Pittsburgh, PA: RWS Publications, 4922 Ellsworth Avenue, Pittsburgh, PA 15213.

[2] Saaty, T. L. (2005). Theory and Applications of the Analytic Network Process. Pittsburgh, PA: RWS Publications, 4922 Ellsworth Avenue, Pittsburgh, PA 15213.

[3] Saaty, T. L. \& Ozdemir, M. (2005). The Encyclicon. RWS Publications, 4922 Ellsworth Avenue, Pittsburgh, PA 15213. 
[4] Buede D. and D.T. Maxwell, 1995. Rank Disagreement: A comparison of multi-criteria methodologies. Journal of Multi-Criteria Decision Analysis 5:1-21.

[5] Saaty, T.L. and L. T. Tran, (2007). On the invalidity of fuzzifying numerical judgments in the Analytic Hierarchy Process, Mathematical and Computer Modelling

[6] Saaty, T.L., and K. Peniwati, (2008). Group Decision Making, Pittsburgh, PA: RWS Publications, 4922 Ellsworth Avenue, Pittsburgh, PA 15213.

[7] Saaty, T.L., and Y. Cho (2001). The Decision by the US Congress on China's Trade Status: A Multicriteria Analysis, Socio-Economic Planning Sciences, 35, 243-252. 